\title{
SYNTHETIC APERTURE RADAR: RAPID DETECTION OF TARGET MOTION IN MATLAB
}

\author{
A Thesis \\ Presented to \\ the Faculty of California Polytechnic State University, \\ San Luis Obispo
}

\author{
In Partial Fulfillment \\ Of the Requirements for the Degree, \\ Master of Science in Electrical Engineering
}

by

Daniel Stephen Kassen

May, 2015 
(C) 2015

Daniel Stephen Kassen

ALL RIGHTS RESERVED 


\section{COMMITTEE MEMBERSHIP}

TITLE:

AUTHOR:

DATE SUBMITTED:

COMMITTEE CHAIR:

COMMITTEE MEMBER:

COMMITTEE MEMBER:
Synthetic Aperture Radar: Rapid

Detection of Target Motion in Matlab

Daniel Stephen Kassen

May, 2015

John Saghri, Ph. D

Professor of Electrical Engineering

Jane Zhang, Ph. D

Professor of Electrical Engineering

Tina Smilkstein, Ph. D

Assistant Professor of Electrical

Engineering 


\begin{abstract}
Synthetic Aperture Radar: Rapid Detection of Target Motion in Matlab

Daniel Stephen Kassen
\end{abstract}

Synthetic Aperture Radar (SAR) has come into widespread use in several civilian and military applications. The focus of this paper is the military application of imaging point targets captured by an airborne SAR platform. Using the traditional SAR method of determining target motion by analyzing the difference between subsequent images takes a relatively large amount of processing resources. Using methods in this thesis, target motion can be estimated before even a single image is obtained, reducing the amount of time and power used by a significantly large amount. This thesis builds on work done by Brain Zaharri and David So. Brain Zaharri successfully created a SAR simulation that accurately models the airborne SAR system capturing data of a target space using the Range Doppler Algorithm (RDA). David So extended this work by adding functionality to determine target velocity in the range and azimuth directions by processing the intermittent data created by the several steps of Brian Zaharri's simulation. This thesis shows further extensions of processing the intermittent data using unique methods. The methods in this thesis successfully demonstrate the ability to quickly and accurately estimate target position, velocity, and acceleration without the need for using multiple SAR images. Target motion in the range direction is detected without using any part of the RDA, while the azimuth direction cuts out several steps, including the range compression phase and the range cell migration correction. Removing these unneeded processing steps dramatically decreases 
target motion data acquisition time. Both Brian Zaharri's and David So's work, along with this thesis, are part of the Cal Poly SAR Automatic Target Recognition (ATR) group of projects, which is sponsored by Raytheon Space \& Airborne Systems Division. Because U.S. military SAR data remains classified, the Cal Poly SAR ATR projects addresses the need to educate researchers on the processing of SAR data.

Keywords: Synthetic, Radar, Digital Image Processing, Matlab 


\section{ACKNOWLEDGMENTS}

I thank the Faculty Advisor for the ATR projects group, Dr. John Saghri, for his help in maintaining this project at Cal Poly, and his continued support and guidance for this project. Raytheon Space \& Airborne Systems Division also deserves recognition for their continued funding and assistance for the multitude of projects created for ATR. Without the perseverance of both Dr. Saghri and Raytheon, these projects would not exist. I thank Brian Zaharri for creating such a robust simulation model on which I was able to develop my code, and David So for demonstrating the possibility of processing the simulation data to extract specific information. Without the work done by these two, my additions would not have come to fruition as they have. Finally, I thank my friends and family for their continued support as I have progressed through this project. 


\section{TABLE OF CONTENTS}

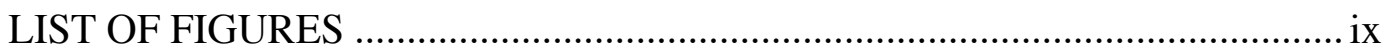

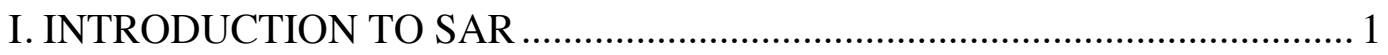

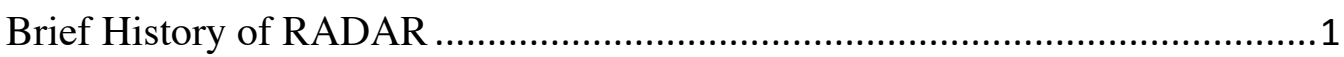

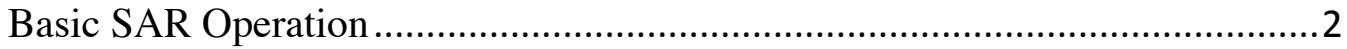

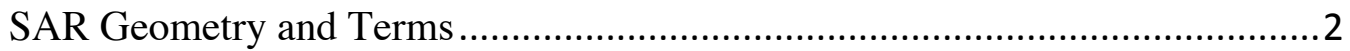

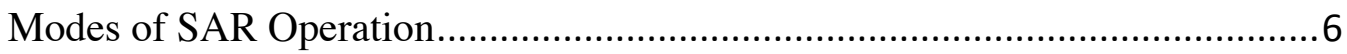

SAR Signal Pulse Transmission and Reception................................................

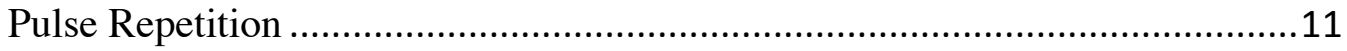

Azimuth Beam Pattern and Doppler Bandwidth ..............................................13

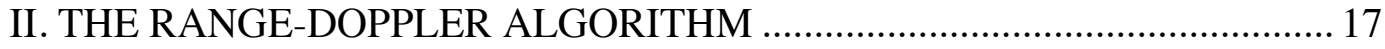

Overview of the Range Doppler Algorithm .................................................18

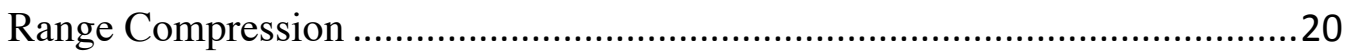

Azimuth Compression and Range Cell Migration Correction ...........................23

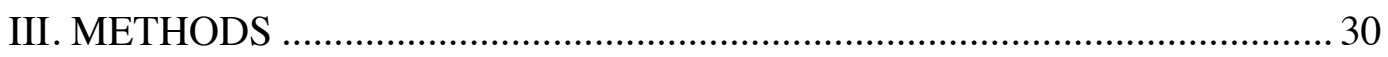

Range Direction Motion Detection .................................................................

Azimuth Direction Motion Detection ................................................................40

IV. SIMULATION RESULTS AND ANALYSIS ……………………................ 47

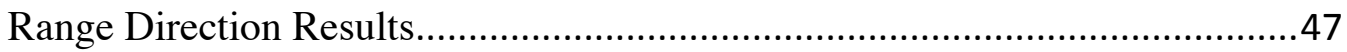

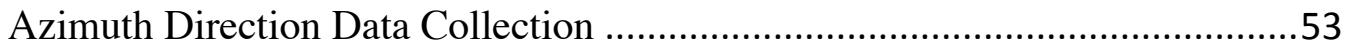

Azimuth Motion Detection Results ................................................................59

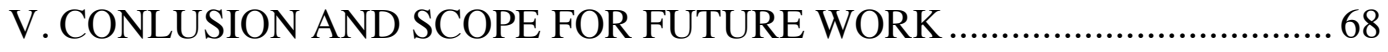

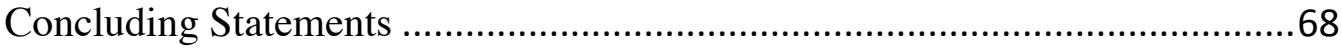

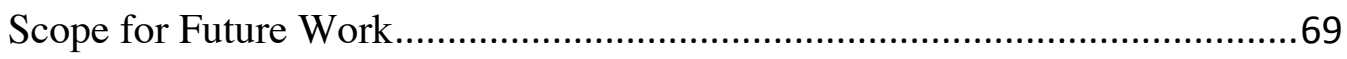

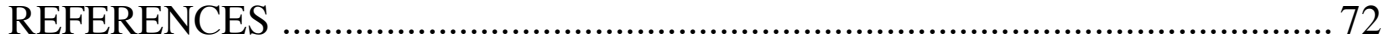

APPENDICES

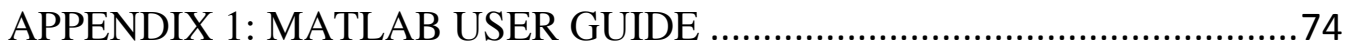

How to use the SAR Looping Script for Testing ........................................... 74

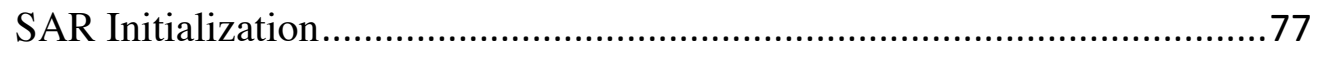

Synthetic Aperture Radar Simulation.........................................................

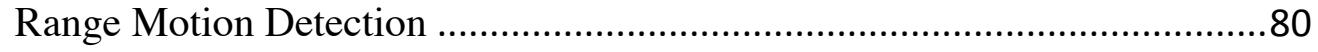

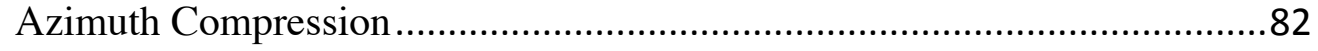

Azimuth Compression Profile Data Collection ............................................ 83

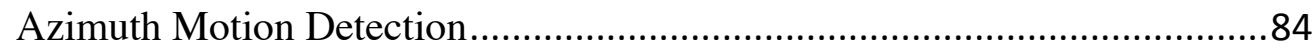


Power Equalization and Range Compression...........................................87

Offline Processing and Compensation ................................................8 88

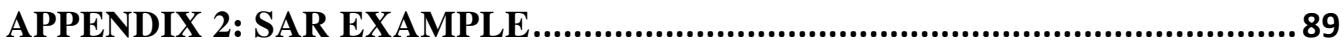

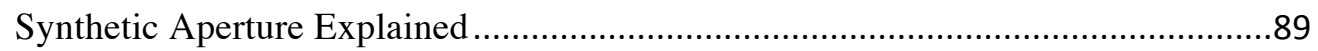




\section{LIST OF FIGURES}

Figure 1: Depiction of the SAR system geometry............................................ 3

Figure 2: Depiction of the "stripmap" mode of SAR ........................................... 7

Figure 3: Pulse train sent by the transmitter ....................................................... 8

Figure 4: Depiction of SAR signal transmission and receiving timing ............... 11

Figure 5: Azimuth beam pattern as the platform approaches and passes the

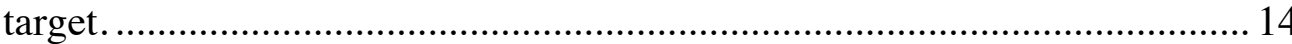

Figure 6: A flowchart depiction of the Range-Doppler Algorithm as performed by the simulation

Figure 7: Example of raw data collected by the SAR system. This image depicts the raw data for a single stationary target located at the center of

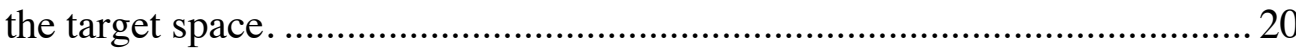

Figure 8: Time domain representation of the matched filter process.

Figure 9: Range compressed data for a stationary target in the center of the target space

Figure 10: Signal returns from a single stationary target in the center of the target space

Figure 11: The Range compressed data brought into the azimuth frequency domain via the y-directional Fourier transform. To the left is the entire data set. To the right is a zoomed view of the target data to show curvature

Figure 12: A depiction from Cumming and Wong depicting how SAR data is saved in memory, showing a relatively exaggerated occurrence of range cell migration.

Figure 13: Range Doppler Data after Range Cell Migration Correction, zoomed in on the relevant target data.

Figure 14: Azimuth matched filter in the sample domain (left) and the frequency domain (right)

Figure 15: Final image in the Range Doppler algorithm for a stationary target

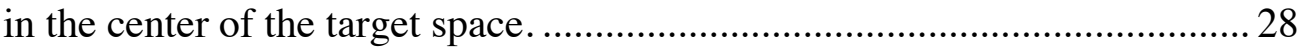

Figure 16: Final target image, zoomed to target ............................................. 28

Figure 17: Steps used for azimuth motion detection. The "final processed SAR image" in this case is without range compression or range cell migration correction.

Figure 18: Range compressed Image for target wait constant $+25 \mathrm{~m} / \mathrm{s}$ velocity .. 32 Figure 19: Raw Data for a target moving with a constant range velocity of +25 $\mathrm{m} / \mathrm{s}$

Figure 20: Target distance form the center of the target space in range bins versus slow time samples 
Figure 21: The difference in range bins between each sample .......................... 36

Figure 22: Raw Data for and accelerating target ........................................... 37

Figure 23: Accelerating Target Location Data ................................................ 38

Figure 24: Accelerating target velocity for each slow time sample..................... 39

Figure 25: Target acceleration for each slow time sample. ............................... 40

Figure 26: Top-down view of the raw data azimuth Fourier transform of the target. These images can be seen as a top-down view of a height map that shows signal strength.

Figure 27: Azimuth Compressed raw data depiction in 3 dimensions to show the magnitude of returns (left), and a profile view of this data looking down range (right)

Figure 28: Azimuth compression data down-range profile view for targets traveling at $0 \mathrm{~m} / \mathrm{s}$ (left) and $50 \mathrm{~m} / \mathrm{s}$ (right).

Figure 29: Actual Target Velocity and Estimated Target Velocity for 1000 iterations. 48

Figure 30: Percent error in estimating target's range velocity

Figure 31: Velocity error in estimating the target's range velocity. 50

Figure 32: Target actual range velocity plotted against the range velocity estimated by the algorithm

Figure 33: Percent error when estimating the target's range acceleration. ........... 52

Figure 34: Acceleration error when estimating the target's range acceleration.... 53

Figure 35: ACP Maximum, Mean, Width, and Azimuth Bin Estimate for a stationary target with each iteration incrementing the position from-190 meters to 190 meters from the center of the azimuth in increments of 0.038 meters.

Figure 36: ACP Maximum, Mean, Width, and Azimuth Bin Estimate for target with each iteration incrementing the velocity from $-50 \mathrm{~m} / \mathrm{s}$ to $50 \mathrm{~m} / \mathrm{s}$ in increments of $0.1 \mathrm{~m} / \mathrm{s}$.

Figure 37: ACP Maximum, Mean, Width, and Azimuth Bin Estimate for target with each iteration incrementing the acceleration from $-25 \mathrm{~m} / \mathrm{s}^{2}$ to 25 $\mathrm{m} / \mathrm{s}^{2}$ in increments of $0.05 \mathrm{~m} / \mathrm{s}^{2}$.

Figure 38: Actual target position and estimated target position for 1000 iterations.

Figure 39: The percent error from estimating Target Azimuth Position............. 61

Figure 40: The error in meters, estimating Target Azimuth Positions. ................ 62

Figure 41: Actual Azimuth Velocity and Estimated Velocity vs. Iteration

Number

Figure 42: Estimated Azimuth velocity percent error 63

Figure 43: Estimated Azimuth Velocity Error vs. Azimuth Velocity 
Figure 44: Actual Azimuth Acceleration and Estimated Azimuth Acceleration v. Iteration Number 65

Figure 45: Estimated Azimuth Acceleration Percent Error v. Azimuth

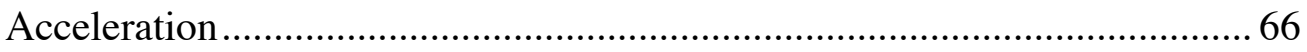

Figure 46: Azimuth Acceleration Error v. Azimuth Acceleration......................... 66 


\section{INTRODUCTION TO SAR \\ Brief History of RADAR}

In the early 1800s, Heinrich Hertz discovered that electromagnetic waves behave similarly to visible light when they reflect off of solid surfaces. The first real practical use of this discovery was implemented in 1904 by German inventor Christian Huelsmeyer, who used reflected electromagnetic waves coming from ships within a $3 \mathrm{~km}$ radius of his transmitter to avoid ship-to-ship collisions [1]. It was not until World War II that detection of objects using electromagnetic waves came into widespread use. The term RADAR, or RAdio Detection And Ranging, was originally coined by the U.S. Signal Corps in 1939 as it developed these systems for the war [2]. RADAR systems originally came into use for measuring the distance to a target in several land- and sea- based applications. It was later discovered that Doppler shifts resulting from these reflected waves could be processed to create high-resolution images of the target area under surveillance. The method used for creating these high-resolution images was termed Synthetic Aperture Radar, or SAR. Originally, SAR data was collected and transformed into images using the principles of Fourier Optics. The advent of digital electronics changed the landscape for this technology, enabling engineers to develop a method for digitally processing these images, known as the Range-Doppler Algorithm, developed by MacDonald Dettwiler and the Jet Propulsion Lab (JPL) in 1978 [3]. Since its inception, scientists and engineers evolved and adapted Synthetic Aperture RADAR to several military and civilian applications such as 
target tracking and identification, terrain imaging, crop health, seabed mapping, medical applications, astronomy, and more.

\section{Basic SAR Operation}

Traditional radar systems used for determining the range to a target begin by transmitting an electromagnetic pulse, measuring the reflected signal returning from the target, and determining the distance to the target via timing and speedof-light calculations. Synthetic Aperture Radar uses this concept, as well as Doppler shifts and the different phases in the return signal, to create an image of the target area; however, instead of using just one pulse, it uses hundreds or even thousands of sequential pulses over a large distance and time span to create a high-resolution image. The following subsections will give light to the physical SAR system.

\section{SAR Geometry and Terms}

Apart from the unintuitive nature of the mathematical concepts behind Synthetic Aperture Radar, the geometry of the process can also be somewhat overwhelming. To assist with understanding the concepts later in this thesis, a list of pertinent terms is described in this section, along with descriptive diagrams of the synthetic aperture radar process are presented in this section. Figure 1 shows the geometrical model used for simulation. 


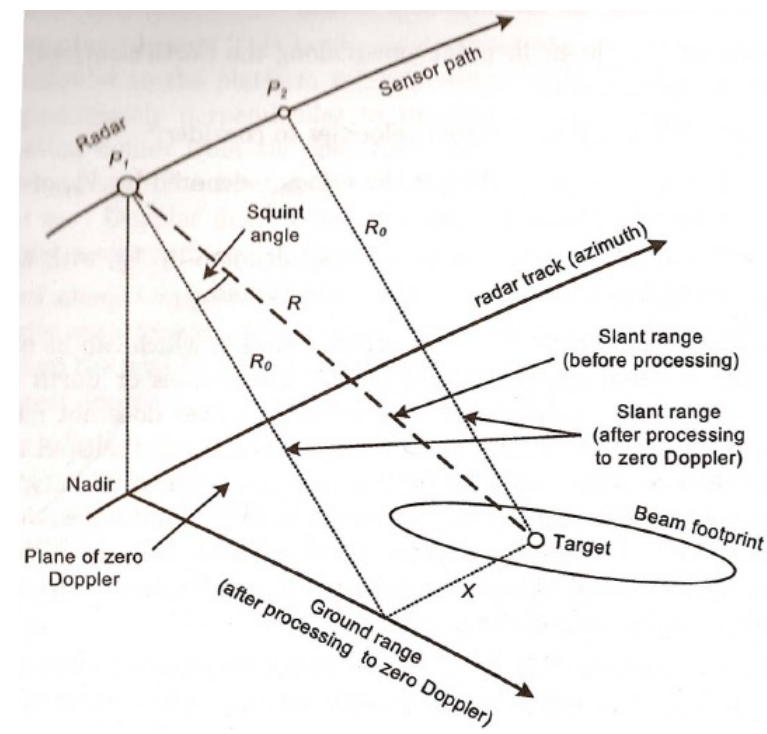

Figure 1: Depiction of the SAR system geometry.

The terms used in the diagram above, as well as terms given rise later in this paper, are listed here:

Range: This term can mean either ground range, i.e. the direction perpendicular to the flight path; or the slant range, which is the physical, absolute distance from the platform (aircraft, spacecraft, etc.) to the target. The ground range in the context of the image space describes the range axis on the image, which is split into discrete sections called range bins. As the index of the range bins increases, so does the associated sample frequency from the up-chirped signal pulse. The highest index range bin (the farthest away) represents the highest frequency in the pulse while the closest range bin represents the lowest frequency. The slant range vector is comprised of three components: the altitude of the platform, the distance of the platform from the point when the target is directly perpendicular to it (zero Doppler shift), and the distance of the target from the radar track. 
Nadir: The point on the earth that is directly below the platform. The nadir traces the radar track (the flight trajectory projected onto the earth) as the platform travels along its trajectory.

Range of Closest Approach, $\mathbf{X}_{\mathbf{c}}$ : The range from the platform to the target changes constantly as the platform moves along its flight path. The range of closest approach describes the minimum distance between the radar platform and the target. It can also be described as the distance between the target and platform when the target crosses the zero Doppler plane, or when the target is directly perpendicular to the platform.

Fast Time (Range Time), $t$ : One of the two time variables used in the SAR process. Fast time refers to the transmission and reception of the signal pulse. It is used as the determining variable for the transmitted pulse's phase. Fast time, along with the instantaneous slant range, allows the division of the range into discrete bins based on the phase of the return signal.

Azimuth: The azimuth direction is the vector that points parallel to the platform velocity vector. Since the simulation assumes a perfectly stable plane, as opposed to the real case where the platform will experience turbulence and slight changes in the flight path, the azimuth can be considered as the direction perpendicular to the range direction and can be used interchangeably with the term 'cross-range'.

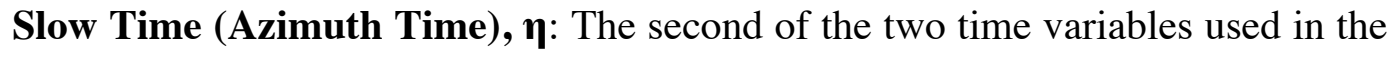
SAR process. Slow time refers to the motion of the radar platform and is used to divide the azimuth into discrete bins. The azimuth time array is a set of discrete 
time values that act as indexes for each of the azimuth bins, and denotes the times that the RADAR pulses are transmitted.

Doppler Shift: The Doppler frequency shift from the Doppler Theory in classical physics. It describes the relative change in frequency experienced by an observer measuring a reflected wave (either sound or electromagnetic) from a target.

Zero Doppler Plane: The constant geometric plane that extends laterally outward from the radar platform. Signals that return from the Zero Doppler plane have a zero Doppler frequency shift.

Squint Angle, $\boldsymbol{\theta}_{\text {sq }}$ : The angle that the slant range vector makes with the zero Doppler plane. The squint angle is zero when the target is at the range of closest approach.

Beam Center Crossing Time: This is the time when the target is illuminated by the center of the azimuth beam pattern. Because the zero Doppler plane is directly perpendicular to the platform velocity vector in the simulation used for this paper, this parameter can also be described as the time when the squint angle is zero, or as when the target is at the range of closest approach.

Pulse Repetition Frequency, PRF: The frequency at which the radar system generates pulses. 


\section{Modes of SAR Operation}

While modern SAR platforms are implemented on various types of vehicles and in different "modes", this paper focuses on the airborne stripmap application of SAR in the context of target tracking. Stripmap SAR is a form of side-looking airborne radar (SLAR) that creates a mapping of the target area as the aircraft moves along its flight path. For this mode of operation, the radar platform is kept in a fixed position; it sends pulses and receives the reflected energy from the pulse, and concatenates the data from this pulse onto the aggregation of all previous pulses. As the radar continues collecting data, it keeps amending the image column by column (each column of the image represents a pulse from the radar platform), creating a long strip-like map of the area in view, hence the name 'stripmap'. In contrast, a different and equally valuable method of airborne SAR is called 'spotlight' mode, for which the radar platform adjusts its looking angle as the aircraft moves along the flight path, such that the aperture is constantly pointed at a fixed plot of land. Stripmap mode SAR is used for surveying a large area, whereas spotlight mode obtains detailed information about a fixed target. Stripmap mode SAR is used for simulation and analysis later in this paper. A representation of stripmap mode can be seen in figure 2 


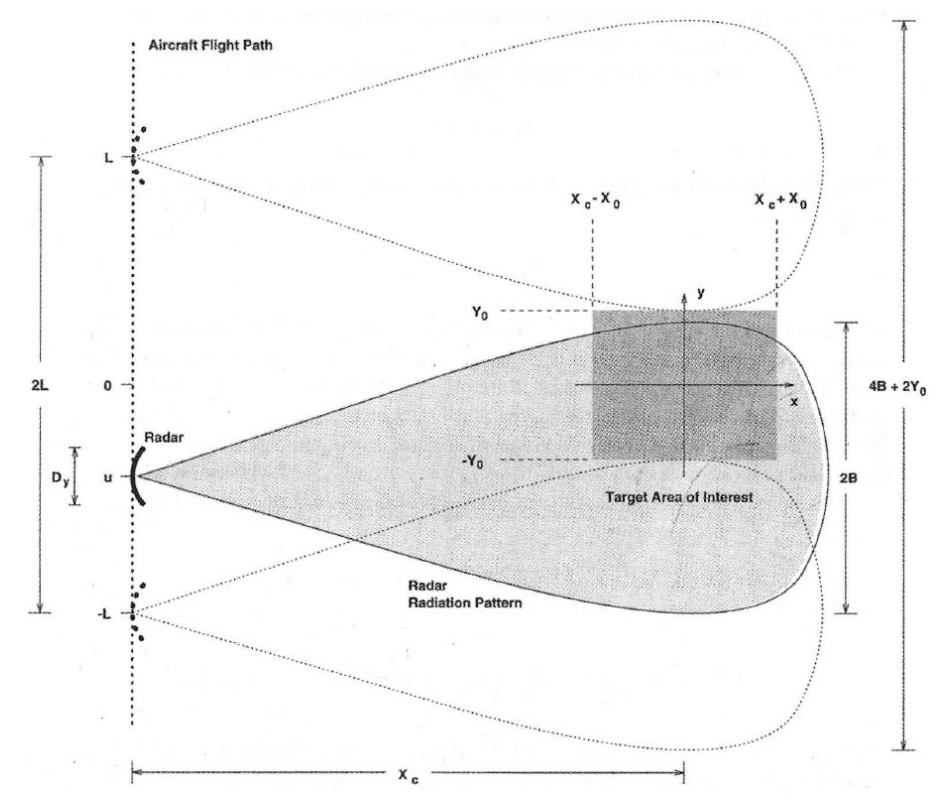

Figure 2: Depiction of the "stripmap" mode of SAR.

\section{SAR Signal Pulse Transmission and Reception}

As mentioned, the SAR system sends and receives several hundreds of sequential pulses throughout the sample period. A single signal pulse is described by the equation:

$$
s_{p u l}(t)=w_{r}(t) \cos \left(2 \pi f_{c} t+\pi K_{r} t^{2}\right)
$$

where $\mathrm{w}_{\mathrm{r}}(\mathrm{t})$ represents the time-domain rectangular pulse generated by the system, $\mathrm{f}_{\mathrm{c}}$ is the carrier frequency, and $\mathrm{K}_{\mathrm{r}}$ is a constant parameter chosen by the system designer known as the chirp rate, and determines the rate at which the phase of each pulse is modulated. The time variable in the equation above, denoted by $t$, is known as fast time or range time. Fast time is used to describe each pulse as it is generated and received and will be described in more detail shortly. A positive 
value of $\mathrm{K}_{\mathrm{r}}$ is called an "up chirp", because the frequency of the pulse increases with time, whereas a negative value is known as and "down chirp" and decreases the pulse's frequency. The simulations used for this paper use a positive value for $\mathrm{K}_{\mathrm{r}}$. The process of sending multiple sequential pulses from the transmitter's perspective is shown in the following figure.

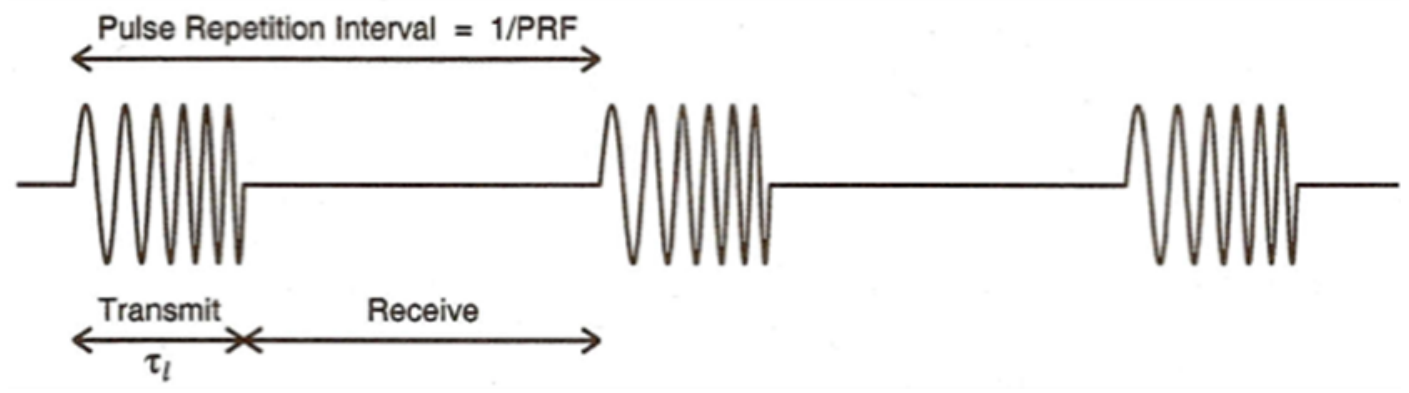

Figure 3: Pulse train sent by the transmitter

The transmitter sends pulses with at the pulse repetition frequency. The pulses last for a few microseconds, after which the system listens for the echoed signals coming from the target space. This figure indicated an up chirped signal because each of the pulses increases in frequency from start to finish.

The chirp rate is linearly related to the bandwidth $B_{0}$ of the system through the equation below:

$$
B o=\left|K_{r}\right| t_{r}
$$

The chirp rate gives variation, either increasing or decreasing, to the frequencies transmitted by each of the pulses. These changes in frequency over time cause 
different sections of the pulse to have a different frequency from the rest of the pulse. Each of these sections reach and reflect sections of the target space, or range bins, at different times. This means that different-frequency sections of the pulse return to the receiver at sequentially increasing times, allowing the system to sample each of these sequential returns from the target space range as they arrive. The number of range bins in the final data is the product of the sampling time and the sampling frequency.

The chirp rate is also inversely proportional to the resolution in the range direction:

$$
\rho_{r}=\frac{\mathrm{c}}{2 B_{o}}
$$

As is true with all digital systems, increasing the bandwidth of the system increases the amount of data it can collect by increasing the range resolution, shortening the distance represented by each range bin.

Before sampling, the return signals are quadrature demodulated to remove the high-frequency carrier, which results in complex magnitude-phase pairs for each sample. The return signals are described by the equation,

$$
\begin{aligned}
s_{r x}(t, \eta)=\sum_{m=0}^{M-1} & {\left[F_{n} w_{r}\left(t-\frac{2 R_{m}(\eta)}{c}\right) w_{a}\left(\eta-\eta_{c}\right) e^{-j 4 \pi\left(\frac{f_{0} R_{m}(\eta)}{c}\right)+j \pi K_{r}\left(t-\frac{2 R_{m}(\eta)}{c}\right)^{2}}\right] } \\
& +n_{m}(t, \eta)
\end{aligned}
$$


Where $F_{n}$ is the reflectivity of the target and is expressed as a constant, $w_{r}$ is the rectangular envelope function; the $\frac{2 R_{m}(\eta)}{c}$ term represents the time delay from the transmitted signal's propagation; $w_{a}\left(\eta-\eta_{c}\right)$ is the function that describes the beam pattern of the transmitter and accounts for the change in amplitude of the received signal with respect to sow time, $\eta$; and $n_{m}(t, \eta)$ is white Gaussian noise added by the system. Note that there are two variables used as input parameters for this equation. The first is fast time, $\mathrm{t}$, and the second is slow time or azimuth time, $\eta$. Slow time relates to the motion of the platform in the azimuth direction for calculating the instantaneous slant range and squint angle. In the equation above, slow time is the reference for when the system sends out pulses and acts as time indices for each fast time pulse. In the equation above, $\eta_{\mathrm{c}}$ represents the time of closest approach or the time when the platform reaches the range of closest approach. Fast time serves to describe the signal pulse as it is generated and received and it is appropriate to consider fast time as a recurring variable that initializes to zero at each pulse transmission.

Each of the signals generated by the SAR system are coherent, meaning that they are theoretically unchanging from pulse to pulse with respect to fast time, and the only defining factor for each pulse is the slow time at which it is transmitted, as well as the beam pattern of the transmitter. For our purposes, slow time is considered to be an array of time samples, or time indexes, over the duration of the sampling time, gives us the pulse transmission times, and allows us to split the target area into azimuth bins. Fast time is a linearly increasing variable that allows 
us to separate each slow time sample, or azimuth bin, into range bins using the fast-time-dependent phase constants in equation 1 above.

\section{Pulse Repetition}

The system transmits, receives, and samples each of these pulses periodically. The rate that this cycle occurs is aptly named the pulse repetition frequency $(P R F)$ or the azimuth sampling frequency, which is also determined by the designer and is limited on the upper bound by the time it takes for a complete signal echo to return, and on the lower bound by the maximum Doppler shifts seen by the radar. Having too high of a PRF would cause returns from separate pulses to overlap and reduce the accuracy of the received data, too low and the system will capture data that is too widely spaced and is unrepresentative of the target area being sampled. Figure 4 below depicts a simplified version of the timing involved in transmitting and receiving pulses.

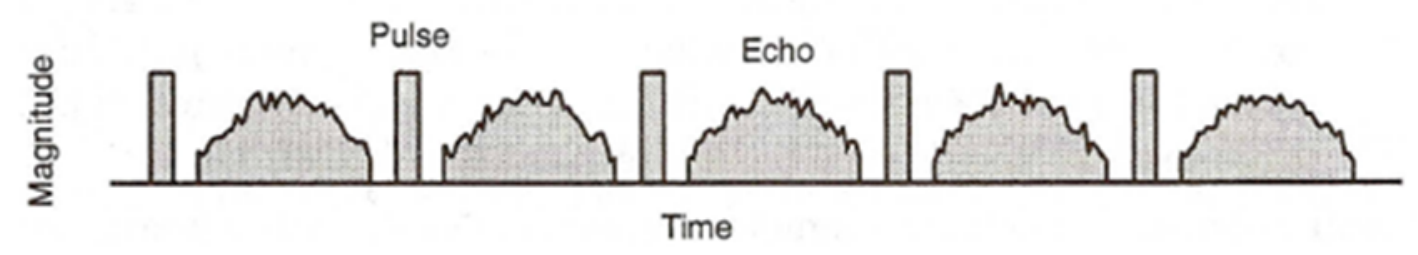

Figure 4: Depiction of SAR signal transmission and receiving timing 
From this figure, it is clear that if the pulses were transmitted at a much higher frequency, the echoes from the returning signals would overlap, causing interference at the receiver and degrading the integrity of the received data.

The PRF is constrained by the time delay in between beginning the transmission of a signal pulse and receiving the return from the furthest range bin. This time delay is equal to the time it takes for the system to transmit the signal with the addition of the back-and-forth propagation time of the signal through the air. The distance over which these signals are transmitted can be approximated as using the instantaneous slant range equation:

$$
R_{m}(\eta)=\sqrt{R_{o_{m}}{ }^{2}+V_{r}{ }^{2} \eta^{2}}=\sqrt{\left(X_{o}+x_{m}\right)^{2}+V_{p}{ }^{2}\left(\eta+\frac{y_{m}}{V_{p}}\right)^{2}}
$$

Since the pulse travels at the speed of light, the theoretical maximum PRF is determined by the inverse of the following equation

$$
t_{P R}=T_{p}+\frac{2 R(\eta)}{c}
$$

Where $T_{p}$ is the pulse transmission time and $c$ is the speed of light. However, since reflections of the pulse do not come solely from the target space, buffer time can be added to the pulse repetition time to account for any stray returns occurring from reflectors outside the target space such that they do not interfere with the next pulse's echo. The lower limit on the PRF that is governed by the maximum Doppler shift is explained in the following section. 


\section{Azimuth Beam Pattern and Doppler Bandwidth}

For each pulse that the radar platform sends out, the target is illuminated; however, the amount to which the target is illuminated varies from pulse to pulse, based on the azimuth beam pattern. As with any antenna, there is directionality involved. This means that the antenna transmits stronger signals in some directions than others, and SAR radar systems are no exception. While beam radiation patterns are different for each system, the pattern used for SAR can be roughly approximated by a sinc function, with a large center lobe and decreasingly smaller side lobes as the target space becomes illuminated by parts of the beam that are further and further from the beam center. The beam pattern is described by the equation:

$$
\rho_{a}(\theta(\eta)) \approx \operatorname{sinc}\left(\frac{0.886 \theta(\eta)}{\beta_{b w}}\right)
$$

Where $\theta(\eta)$ is the instantaneous squint angle and $\beta_{b w}$ represents the radar beamwidth. These parameters are described by the equations:

$$
\begin{gathered}
\theta_{s q}(\eta)=\tan ^{-1}\left(\frac{V p\left(\eta-\eta_{c}\right)}{R_{o}}\right) \\
\beta_{b w}=\frac{0.886 \lambda}{L_{a}}
\end{gathered}
$$

The beamwidth is inversely proportional to the length of the physical antenna. A smaller beamwidth means that the system is gathering information on a smaller space (e.g. higher resolution). Increasing the antenna decreases the beamwidth, ultimately increasing the resolution of the system. 
Because the signal propagates to and from the target, the received signal strength is given by the square of the beam pattern:

$$
\omega_{a}(\eta) \approx \rho_{a}^{2}\{\theta(\eta)\} \approx \operatorname{sinc}^{2}\left(\frac{0.886 \theta(\eta)}{\beta_{b w}}\right)
$$

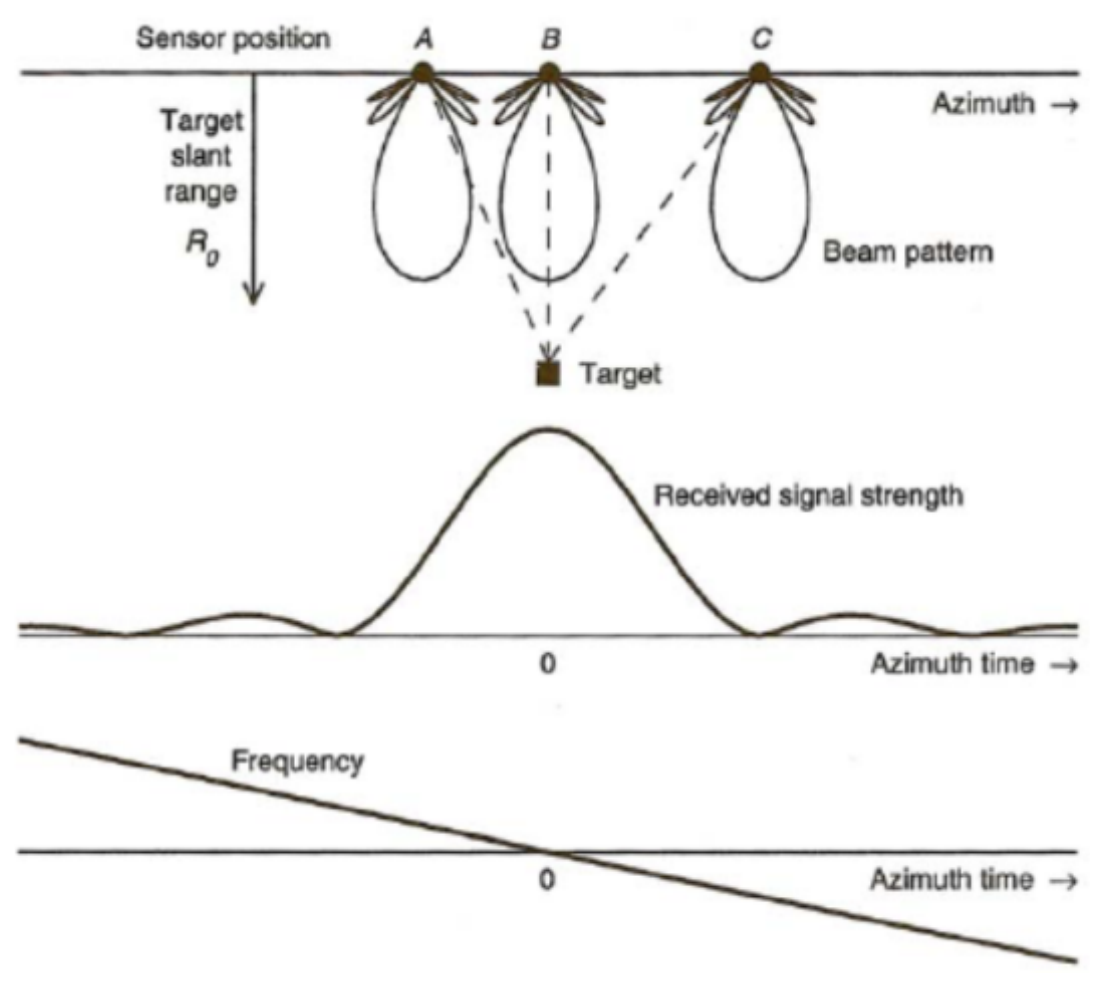

Figure 5: Azimuth beam pattern as the platform approaches and passes the target.

The figure shows an approximate radiation pattern for a SAR system. It represents a polar sinc function whose origin is located at the physical antenna. The peak of main lobe is considered the beam center point, depicted in the figure to point 
directly perpendicular to the platform velocity vector. This means that the beam center is located on the zero Doppler plane, which is the case assumed for the simulation. At the time of closest approach, when the target is directly on the beam center, the return from the target reaches its maximum, as seen from the $w_{a}\left(\eta-\eta_{c}\right)$ term in the received signal equation above. When the target is either in front of or behind the platform along the azimuth, the pulses sent from the radar illuminate it with the side lobes of the beam pattern producing lesser local maxima. The waveform in the center of the figure shows a sketch of the aggregation of all of the return pulses. As expected, the returns form a sinc-like function, as described earlier. The waveform on the bottom of the image shows the Doppler shift seen by the SAR system as it observes the target. At the beginning of sampling, the Doppler shift is positive and at its highest, as the velocity vector towards the target is largest. As the platform approaches the azimuth position of the target, the velocity vector towards the target and the Doppler shift seen by the platform both decrease. The Doppler shift is exactly zero when the target lies on the zero doppler plane (i.e. the beam center) and continues to negatively grow increasingly as the platform passes by the target. The maximum Doppler shift seen by the radar, also known as the Doppler bandwidth, is described by the equation:

$$
\Delta f_{\text {dop }}=\frac{2 V_{p} \cos \theta_{s q, c}}{\lambda} \theta_{b w}
$$

Since $\cos \theta_{s q, c}=1$, because the squint angle of closest approach is zero, and from our synthetic aperture length calculation, $\theta_{b w}=\frac{0.886 \lambda}{L_{a}}$, 


$$
\Delta f_{\text {dop }}=0.886 \frac{2 V_{p}}{L_{a}}
$$

The Doppler bandwidth is important to the SAR system because it puts a lower limit to the pulse repetition frequency. Setting the PRF too low will cause aliasing in the azimuth direction. An oversampling factor of 1.1 to 1.4 of $\Delta \mathrm{f}_{\text {dop }}$ is typically used to avoid this.

The Doppler bandwidth is also used to calculate the resolution in the azimuth direction:

$$
\rho_{a}=\frac{0.886 V_{p} \cos \theta_{s q, c}}{\Delta f_{\text {dop }}}
$$

Making the same assumption as before $\left(\cos \theta_{s q, c}=1\right)$,

$$
\rho_{a}=\frac{0.886 V_{p}}{\Delta f_{\text {dop }}}=\frac{L_{a}}{2}
$$

This is the usual quoted value for the azimuth resolution and is independent of range, velocity, and wavelength. 


\section{THE RANGE-DOPPLER ALGORITHM}

After collecting the raw SAR data, a multiple-step processing algorithm called the Range Doppler Agorithm (RDA) is imposed to extract useful information about the target space. There are no discernable features to the raw data image that would tell us anything about target location, identity, or trajectory. This is where

the SAR system moves from the physical realm to the digital signal processing realm via the Range-Doppler algorithm. Because this paper is about extracting motion data from targets in motion, it should be noted that the Range-Doppler algorithm is used primarily for the imaging of stationary targets, and that moving targets produce final images that do not meaningfully represent the target space. The next chapter will provide more insight into this claim. For reference, relevant radar and RDA terms are defined in Appendix 2.

Target Space: Refers to the physical space being sampled that is illuminated by and reflects the signals generated by the radar platform.

Signal Space/Raw Data: The raw data is the image information collected by the radar system before any image processing operations are performed. The signal space simply refers to the image that represents all of the data collected during sampling.

Matched Filter/ Reference Signal: The matched filter is used to clean up the raw data. There are two matched filters used in the RDA, one for the range direction and one for the azimuth direction. A matched filter represents the ideal returns 
from a point target when it is on the zero Doppler plane. The reference signal is the time domain representation of the matched filter.

Range Compressed Space: After the matched filtering process in the range direction, the data is brought into the range compressed space. At this point in the RDA, the artifacts present in the range direction have been removed by the rangedirection matched filter. This stage of the RDA refers to the final image before it has been filtered in the azimuth direction.

Range Doppler Signal/Range Doppler Space: The range Doppler space refers to the Range Compressed space after performing a Fourier transform in the azimuth direction. In this stage, the range axis is still in samples, like the raw data, but the azimuth direction is in frequency. The frequency values in this stage represent the Doppler shifts for each range bin. After performing Range Cell Migration Correction (RCMC for short), explained later, this signal is multiplied with the azimuth matched filter and brought back to the sample domain, revealing the final image.

\section{Overview of the Range Doppler Algorithm}

The algorithm takes advantage of directional Fourier transforms and matched filtering to clean the noise and extra artifacts from the data collection, leaving behind a relatively clear image of the target. The matched filtering is the main aspect of the RDA. Instead of performing complicated convolutions in the sample domain, the matched filter signal and the raw data are brought into the frequency 
domain through a one dimensional Fourier transform. Once this is done, the two frequency domain signals are multiplied together, which is the mathematical equivalent of performing convolution in the sample domain. Afterwards an inverse Fourier transform is imposed on the result, bringing the image back to the sample domain. This process occurs for both the azimuth and range directions, a range cell migration correction operation in between the range filtering and azimuth filtering to fix errors caused inherently by the geometry of the data collection. The order of operations performed in the RDA is depicted in figure 6 .

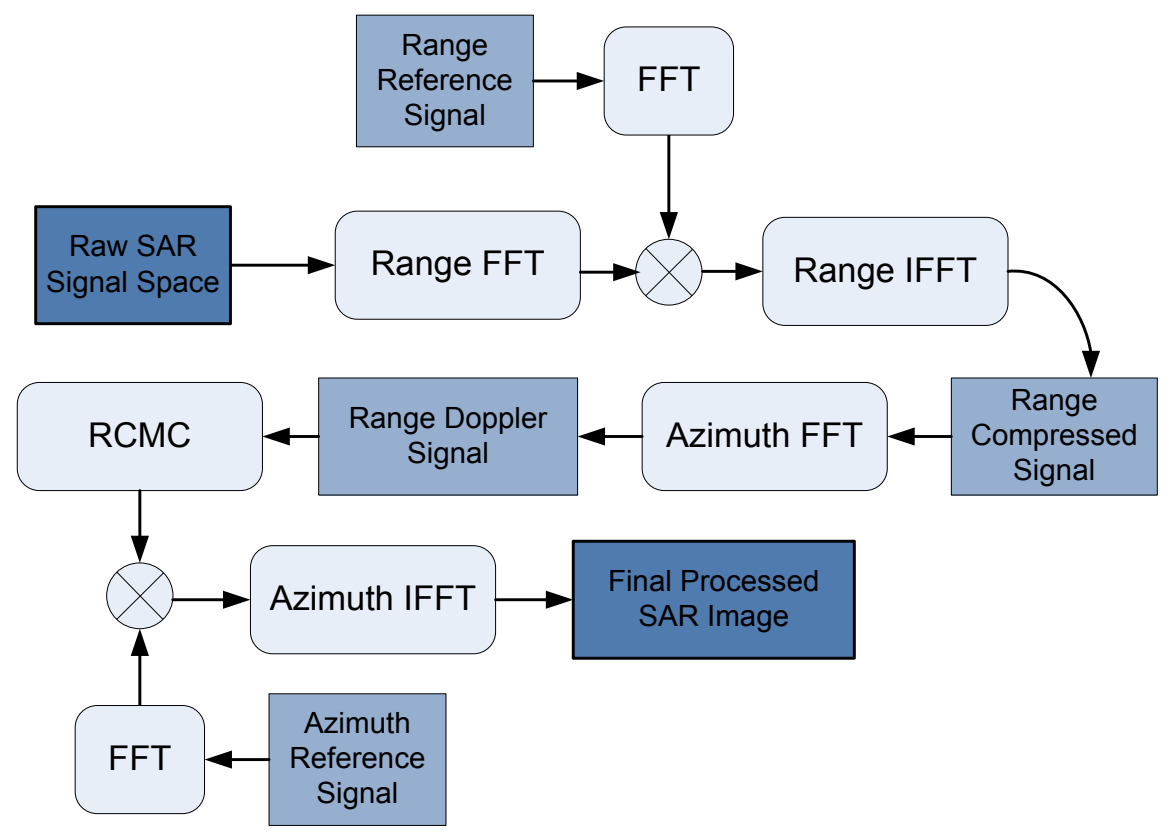

Figure 6: A flowchart depiction of the Range-Doppler Algorithm as performed by the simulation 
The process takes in the raw data seen in figure 7 below. As mentioned earlier, the raw data gives us no discernable features of, or useful information about the target space, other than the existence of a target (if there were no target in the simulation used to produce this image, it would appear to be blank).

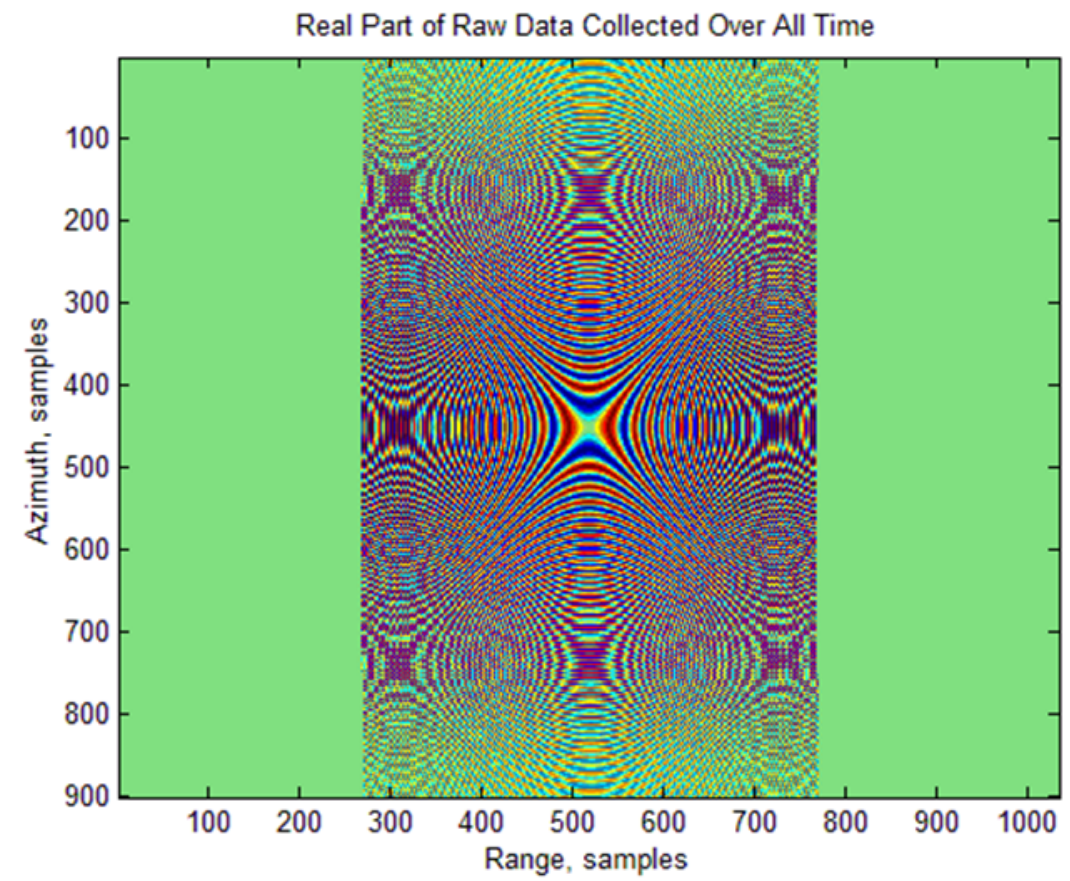

Figure 7: Example of raw data collected by the SAR system. This image depicts the raw data for a single stationary target located at the center of the target space.

\section{Range Compression}

The first step of the RDA process is to perform a one-dimensional Fourier transform in the range direction. The resultant image is in frequency domain for the range direction, and in sample-domain for the azimuth direction. A matched 
filter, which contains the information of the Zero-Doppler signal return in an ideal, noiseless target space, is then multiplied with this signal in the frequency domain, removing the noise and artifacts caused by non-idealities in the sampling. The process of matched filtering in the time domain for a signal pulse is shown in figure 8 .

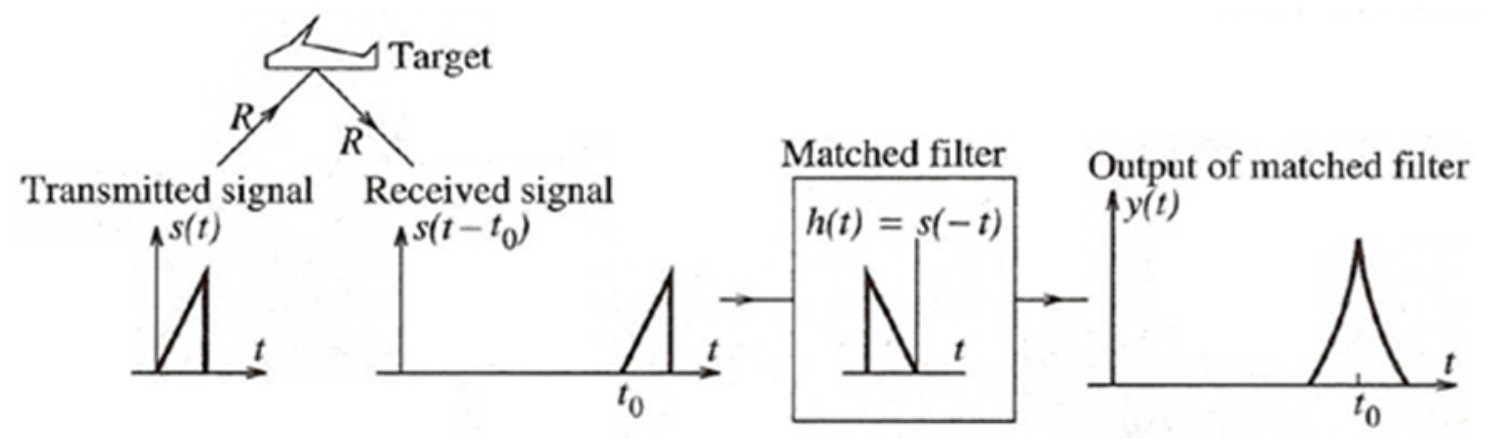

Figure 8: Time domain representation of the matched filter process.

The matched filter is the reverse-time transformation of the time domain reference signal, as shown above. The reference signal for this simulation is representative of the return signal from a point target located at the center of the target space when it crosses the zero Doppler plane. Using Fourier transforms, this signal is taken into the frequency domain with the raw data where the two are multiplied to filter out noise and artifacts. This process is known as convolution. An inverse Fourier transform is then performed in the range direction to bring the data back to the sample domain. An example result of the matched filter operation is shown in the figure 9 below. This result is known as the range compressed data. This simply means that the noise has been removed for the range direction and the 
amount of data has been compressed to include only the pertinent information about the target.

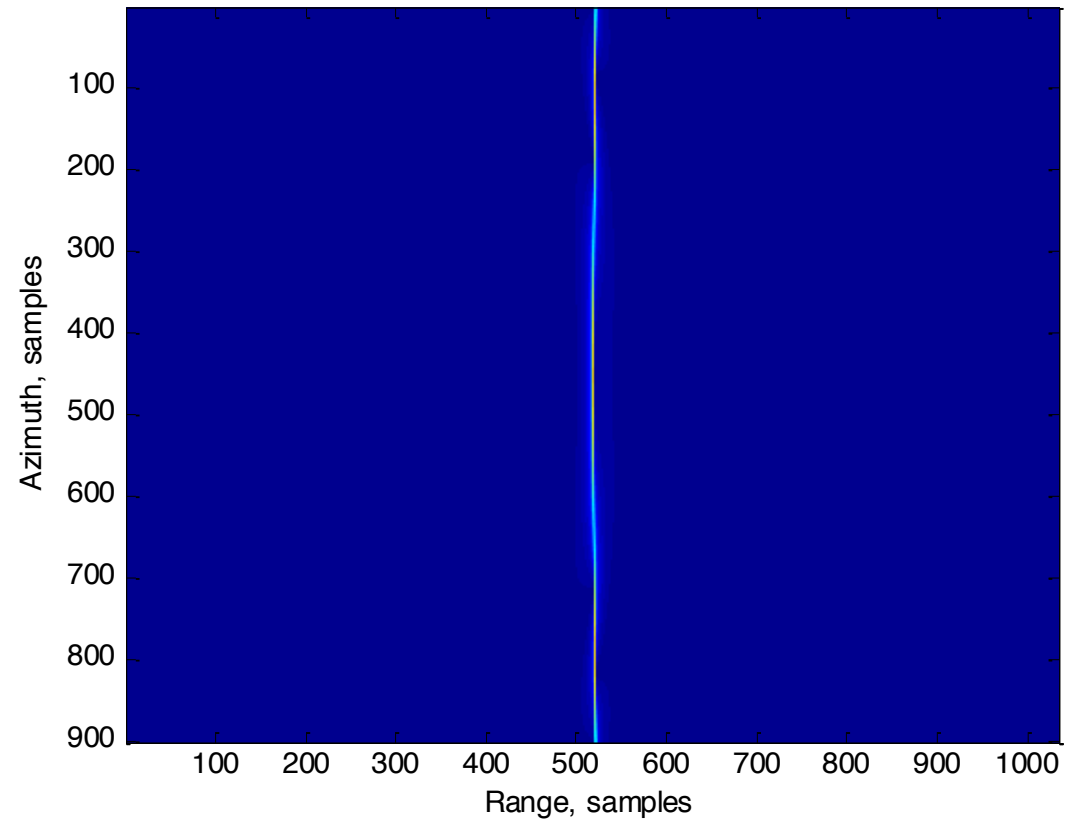

Figure 9: Range compressed data for a stationary target in the center of the target space

Although the image appears as a single line, the line depicts the signal return from the target for each sample taken by the SAR system. Tracing the values along this line results in the previously mentioned $\operatorname{sinc}^{2}$ function that can be seen below in figure 10 . 


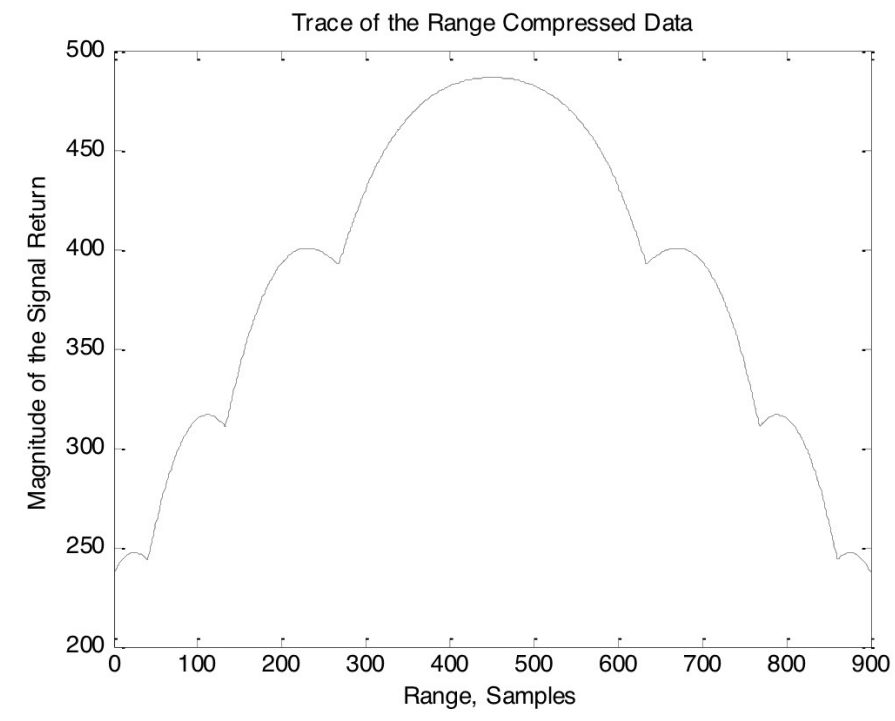

Figure 10: Signal returns from a single stationary target in the center of the target space

\section{Azimuth Compression and Range Cell Migration Correction}

The next step in the process is the application of the one-dimensional Fourier transform in the azimuth direction. The resulting image is that in the Range Doppler Space which represents the Doppler shifts seen by the platform. To start, the range compressed data is brought into the y-frequency domain via the $y$ directional Fourier transform. 

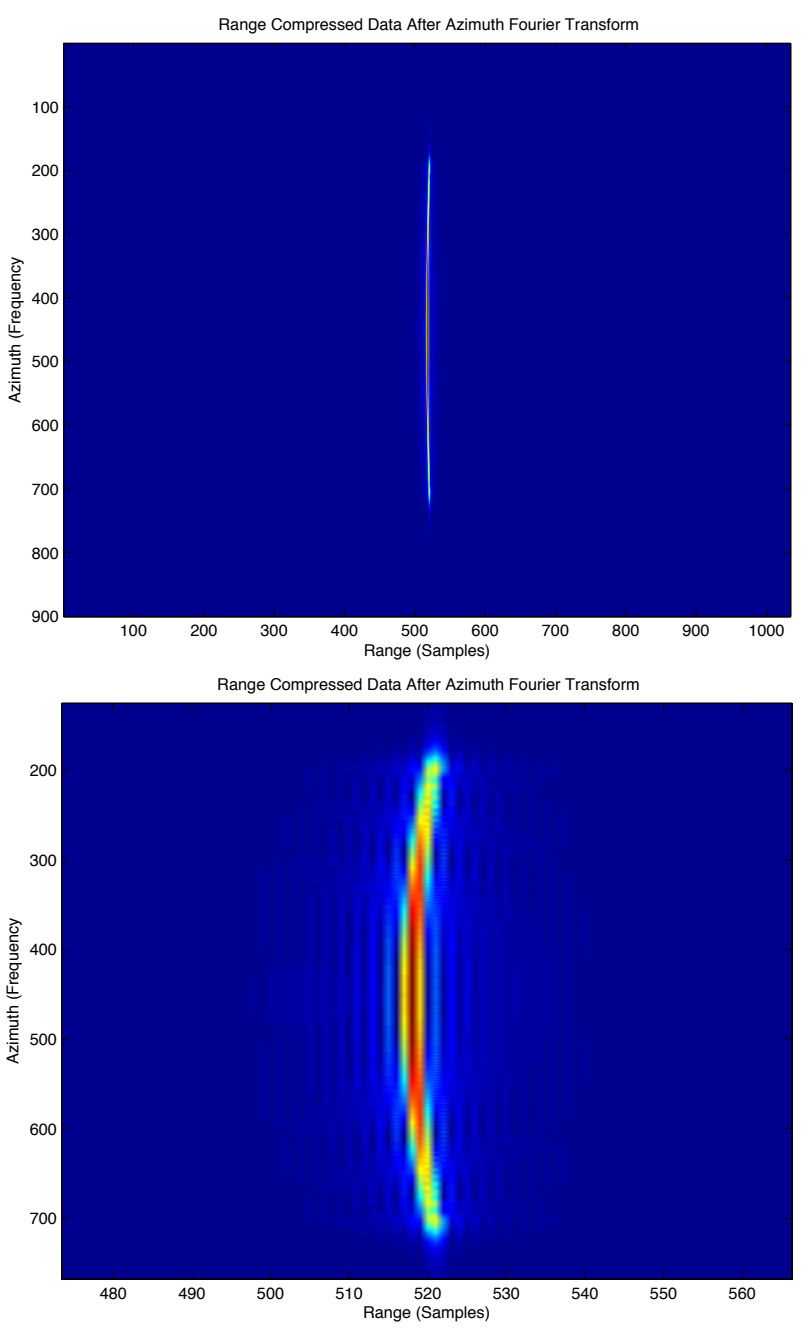

Figure 11: The Range compressed data brought into the azimuth frequency domain via the y-directional Fourier transform. To the left is the entire data set. To the right is a zoomed view of the target data to show curvature.

Following the range compression method, azimuth compression is also needed, but multiplying with a matched filter immediately will cause errors due to a remediable phenomenon called range cell migration. After performing the $y$ direction Fourier transform, it would appear as though the data is parabolically warped. In other words, a line we desire to be straight appears curved. Even 
though the platform moves in a straight line, the target space remains stationary, and there are slight changes in the instantaneous slant range during the time of flight and the percieved distance to the target space changes with each slow time sample, which is apparent in the curved data. In other words,

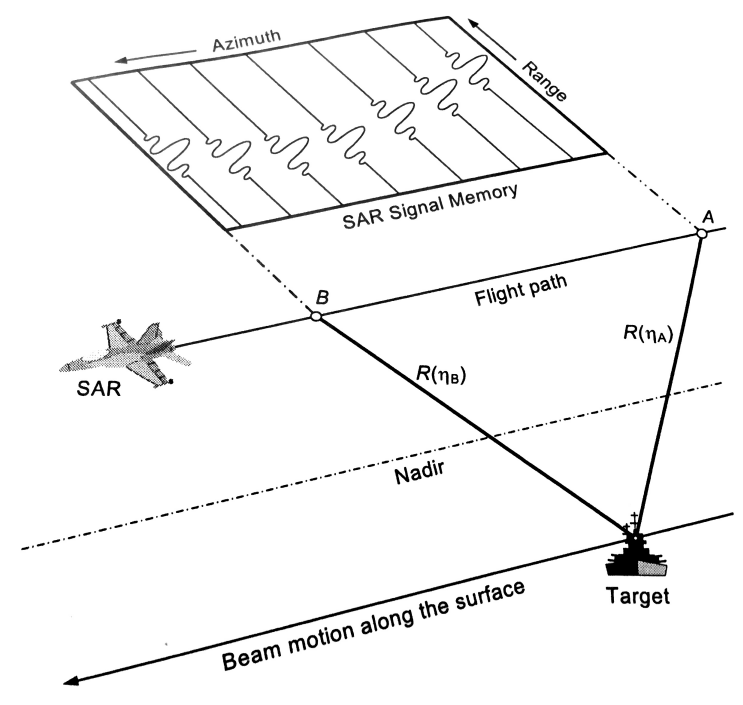

Figure 12: A depiction from Cumming and Wong depicting how SAR data is saved in memory, showing a relatively exaggerated occurrence of range cell migration.

This curvature is inherently present in the method of airborne and spaceborne SAR systems and cannot be physically avoided. The method used in the RDA to account for tis migration is called Range Cell Migration Correction (RCMC). At any point during sampling, the amount of migration in meters can be found with respect to the azimuth frequency: 


$$
\Delta R\left(f_{a}\right)=\frac{\lambda^{2} \mathrm{R}_{\mathrm{o}} f_{a}^{2}}{8 \mathrm{v}_{\mathrm{p}}{ }^{2}}
$$

For each azimuth frequency sample, the amount by which the associated row of pixels must be shifted is calculated using the equation above. After RCMC, the data appears closer to what we expect, although it does not mathematically account for all of the geometric nonidealities.

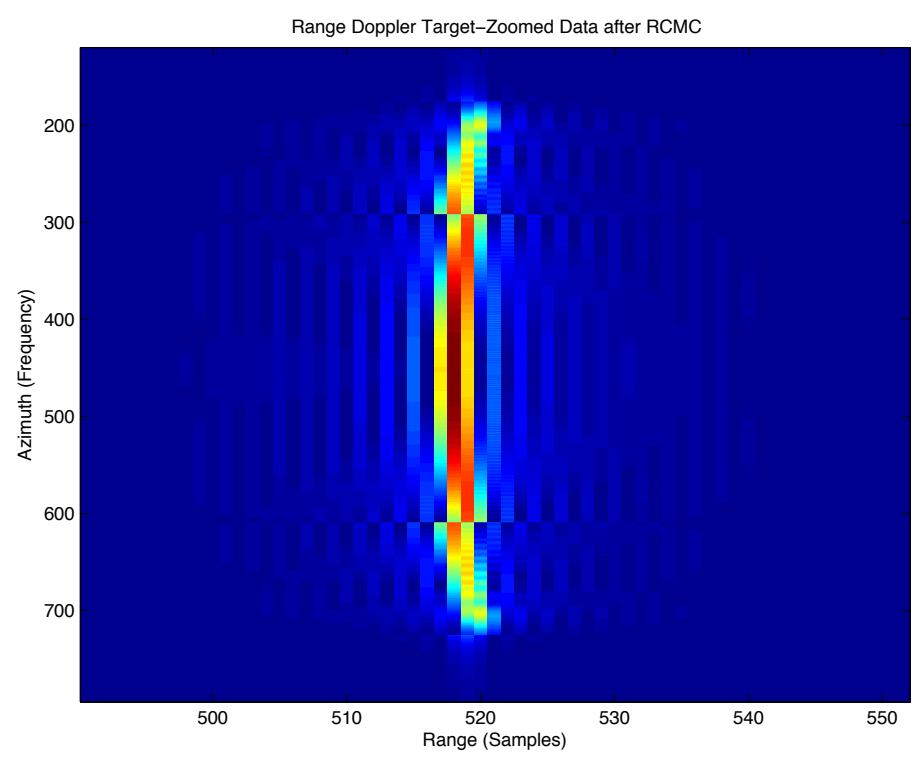

Figure 13: Range Doppler Data after Range Cell Migration Correction, zoomed in on the relevant target data.

The next step in the algorithm is the azimuth matched filter multiplication. A reference signal is defined as the complex signal return from a point target centered in a noiseless environment, mathematically represented by the equation:

$$
S_{m b 0}=e^{j \pi K_{a} \eta^{2}(P R F * d u r+1)}
$$


With its spectrum, or the azimuth matched filter, represented by:

$$
F\left\{S_{m b 0}\right\}=F\left\{e^{j \pi K_{a} \eta^{2}(P R F * d u r+1)}\right\}
$$
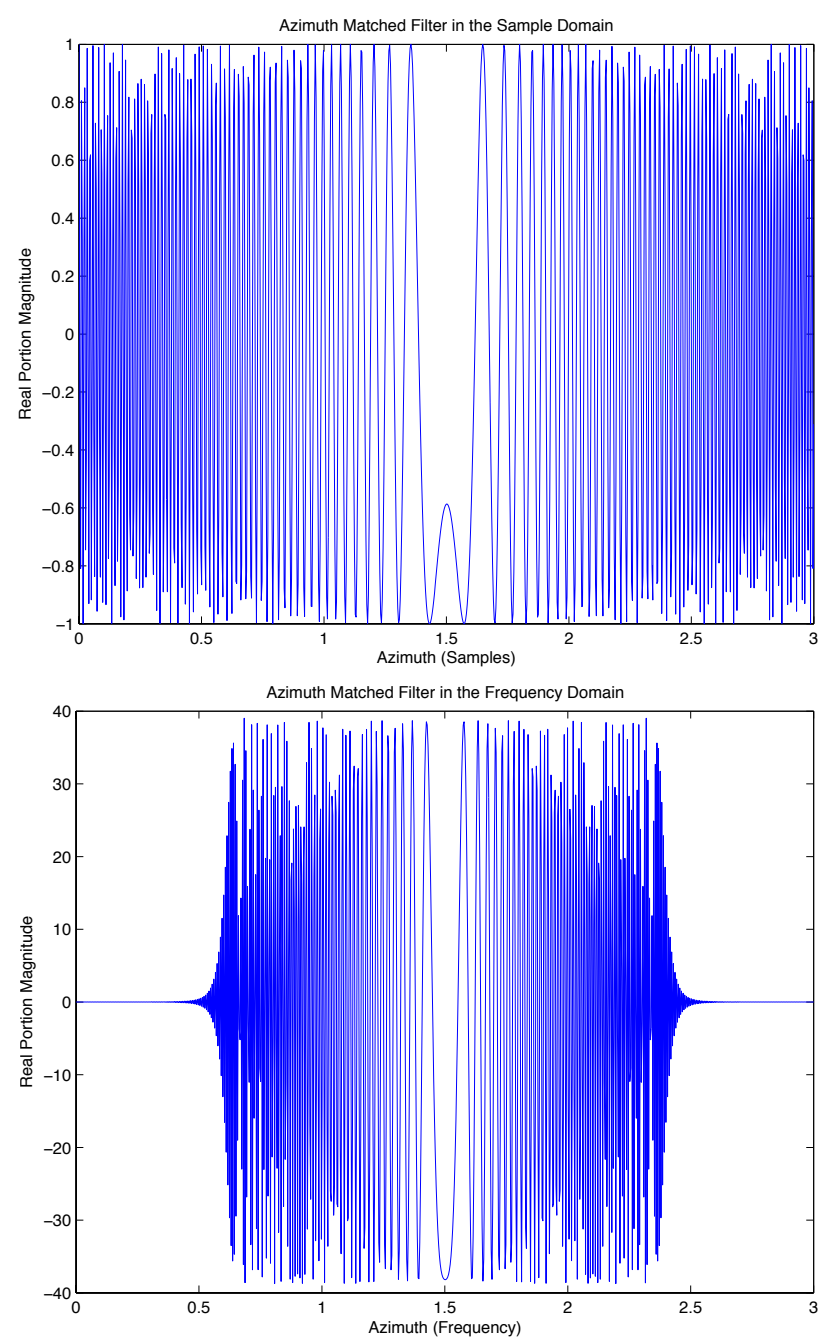

Figure 14: Azimuth matched filter in the sample domain (left) and the frequency domain (right)

The next and final step is to bring the data back into the sample domain via the azimuth inverse Fourier transform, completing the azimuth compression, resulting in and image of the target: 


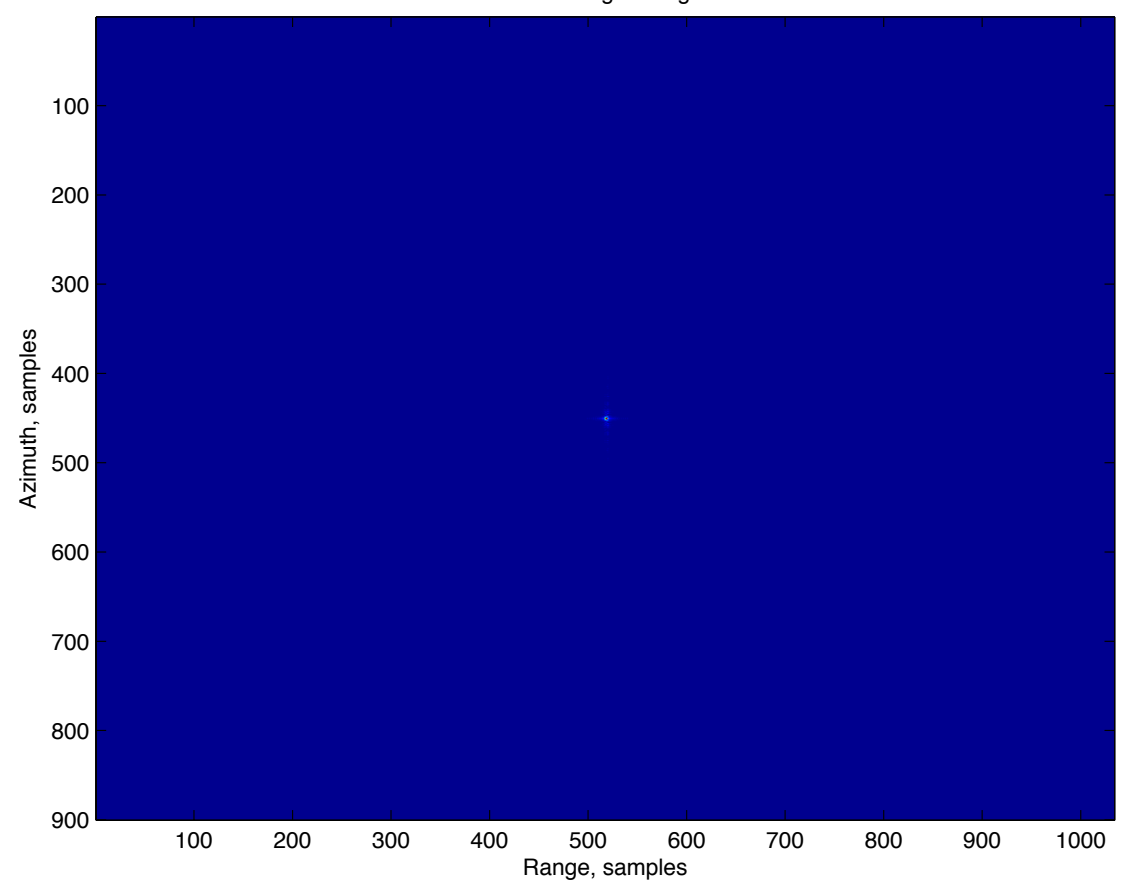

Figure 15: Final image in the Range Doppler algorithm for a stationary target in the center of the target space.

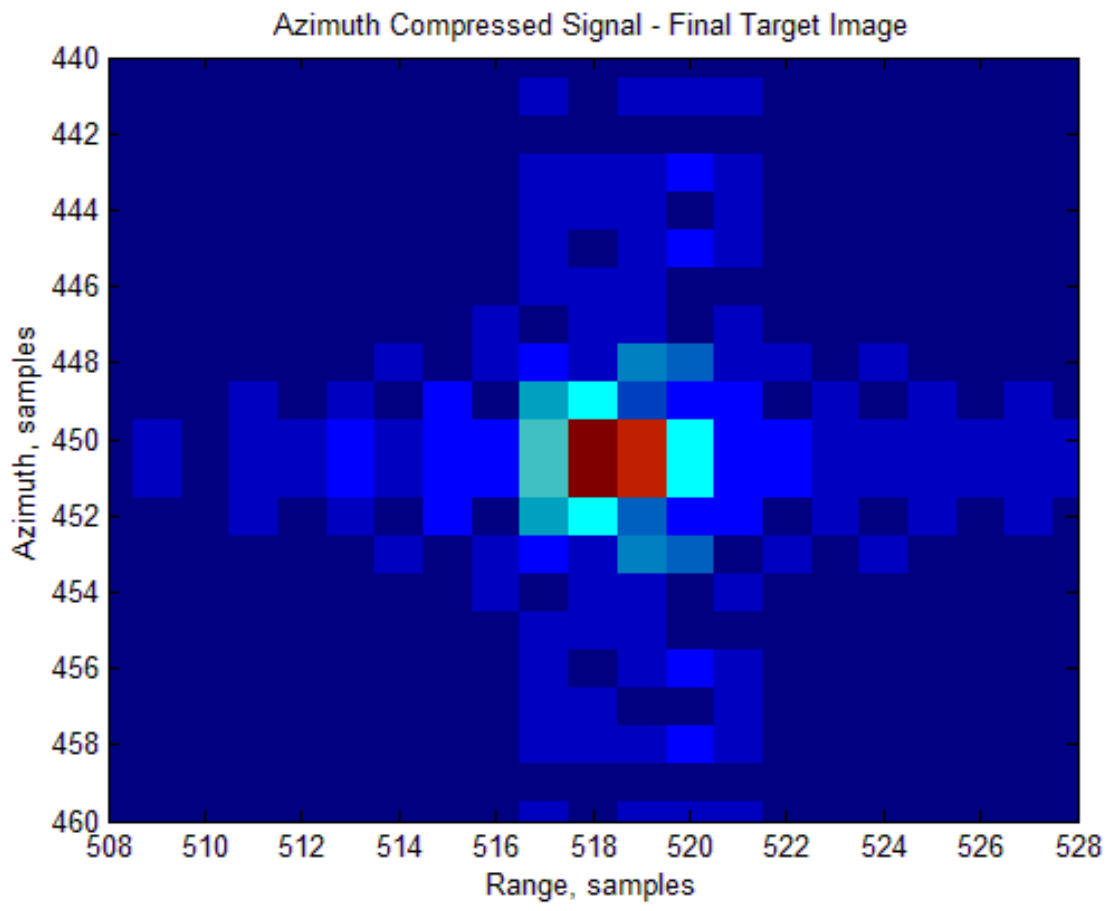

Figure 16: Final target image, zoomed to target 
Note that thesis images show a stationary target. Because the target appears so small in figure 15 , (located To reiterate, the RDA is primarily used for the imaging of stationary targets. Figure 16 was created to show the result of imaging a single point target. The image does not show a single point as we expect, and it may be possible to adjust the simulation parameters to increase the resolution of the simulation such that the final resulting image shows a better representation of the target, but this is beyond the scope of this paper and is discussed in the future work section. This paper discusses the ability to detect targets in motion and will use portions of the RDA in a customized order and excludes entirely some of the algorithm's original steps. 


\section{METHODS}

As stated in the preceding chapter, the Range Doppler-Algorithm is meant primarily for imaging stationary targets. Performing the algorithm on raw data collected from a moving target produces unrepresentative images of the target space. The distortion caused by target motion can be compensated to produce more accurate images, but only if the target moves with constant velocity. The methods described in this section are based loosely on certain elements of the Range-Doppler algorithm, using only certain parts of the algorithm to extract motion information. Referring back to the Range-Doppler flowchart above in figure 6, the algorithm can be broken up into the sub operations described in each block and rearranged to produce different outcomes.

For the motion detection in the range direction, the RDA was avoided entirely. For the azimuth direction, some portions of the RDA were used, but many were skipped to save processing resources and time. The figure below shoes the steps used for the azimuth motion detection:

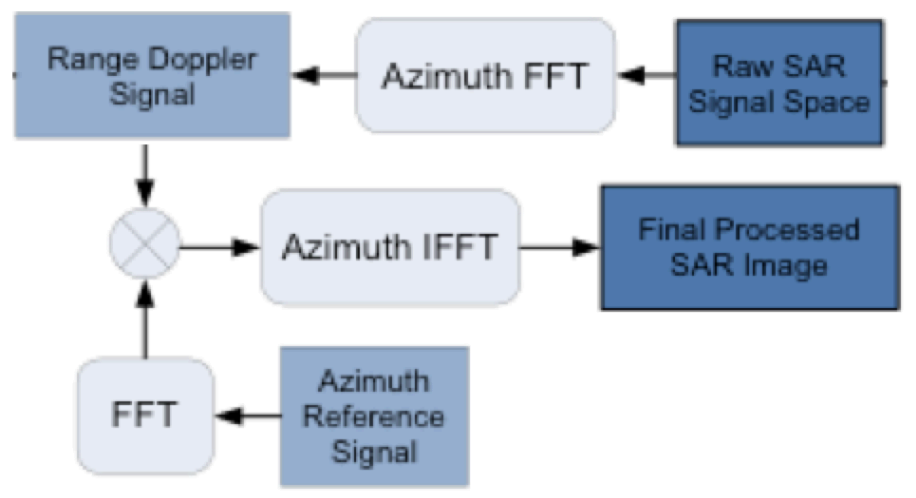

Figure 17: Steps used for azimuth motion detection. The "final processed SAR image" in this case is without range compression or range cell migration correction. 
It is important to note that in order to correctly add in the functionality for a target to accelerate, slight modifications were made to the SAR process such that the velocity would be read in as a vector of varying numbers, where as a constant velocity would be a vector of the same size with a constant value in each element of the vector. This modification can be observed in the Matlab User Guide in Appendix 1.

\section{Range Direction Motion Detection}

Since its inception, radar has been used for determining the distance from the radar platform to a target. It works by detecting when the pulse echo returns from the target. It makes sense that creating an image of the return pulses from a moving target would show the pulse moving in accordance with the target's velocity, and thus the motion of the target can be determined. This fundamental property of radar systems is directly applicable to this application.

Referring to the figure from the Range Compressed Data section in the previous chapter, the image shows a vertically straight line, depicting a target that is not in motion in the range direction, $\mathrm{X}$ (a target moving strictly in the azimuth direction would also produce a vertical straight line). If a target were to move in the range direction, the shape of this line would change accordingly. Previous work showed that the velocity of a target can be extracted from this point in the RDA if the target moves with a constant velocity. The figure below represents a target 
moving in the positive range direction with a constant velocity of +25 meters per second.

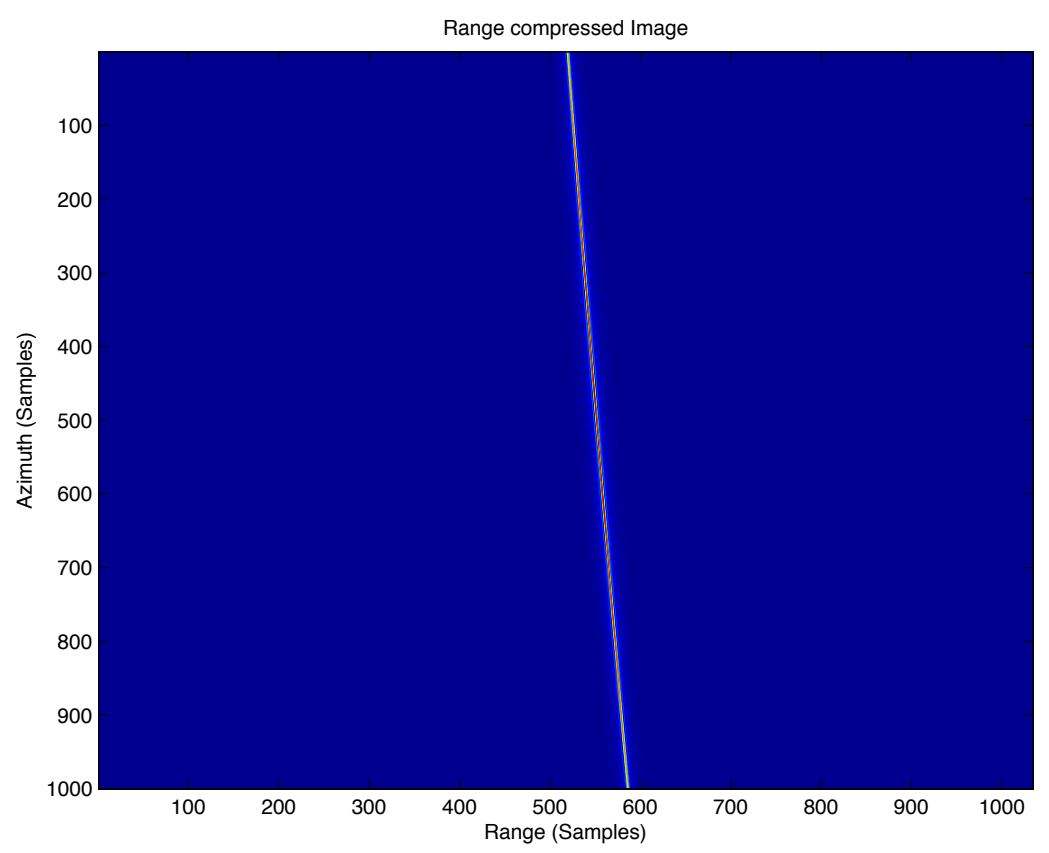

Figure 18: Range compressed Image for target wait constant $+25 \mathrm{~m} / \mathrm{s}$ velocity

Note here that the top row of the image represents the first slow time sample taken and the bottom row of the image represents the last slow time sample taken. Because the target moves with a constant velocity, it shows in the figure that its position changes linearly, and the returned echoes move in accordance with the target motion. Simply finding the slope of this line and converting from samples using a single conversion will give the velocity of the target:

$$
v_{t}=\frac{\Delta_{\text {range }}}{\Delta_{\text {azimuth }}} *\left(\frac{P R F * \rho_{\text {azimuth }}}{V_{p}}\right) * 100
$$

Where $\mathrm{v}_{\mathrm{t}}$ is target velocity, $\Delta_{\text {range }}$ is the change in number of range bins, $\Delta_{\text {azimuth }}$ is the change in number of azimuth bins, $\rho_{\mathrm{a}}$ is the azimuth resolution of the system, 
$\mathrm{PRF}$ is the pulse repetition frequency, and $\mathrm{V}_{\mathrm{p}}$ is the radar platform velocity. While this method does provide anaccurate velocity estimation for a target with unchanging velocity, it does not perform well for targets with considerable acceleration. What's more, this method does not require Range Compression. The same slope can be observed and extracted from the raw data, eliminating the need for the only computationally intense operation in this method.

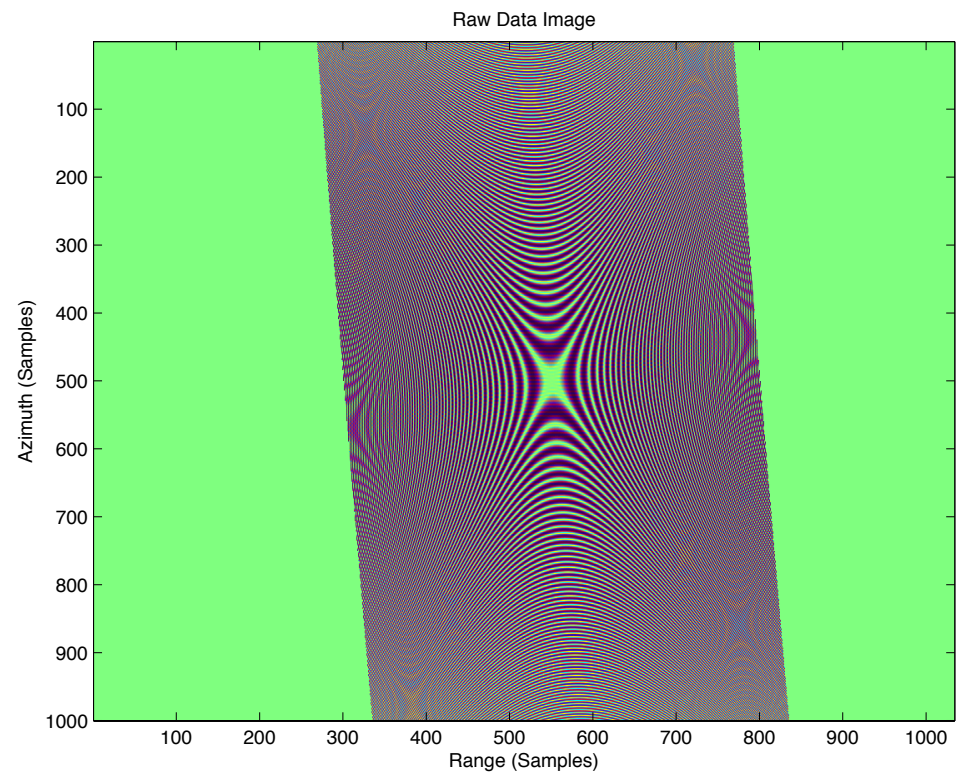

Figure 19: Raw Data for a target moving with a constant range velocity of $+25 \mathrm{~m} / \mathrm{s}$

The data represented in figure 12 appears slanted when compared to the previously shown range compressed data, due to the target's positive velocity. The solid green portions represent irrelevant background data whereas the colorful section shows the signal echoes returned from the target. Each azimuth bin, or slow time sample, in the image corresponds to a row of pixels. In this 
image, we can see that each row of pixels contains two solid green sections on either side and the target echoes. Finding the index of the center of the colorful section in each row gives the target's approximate location (range bin) for each slow time sample. This means that this image contains the approximate location of the target at any time during sampling. The approximate range locations are in discrete range values and appear stepped as in the figure below. Because the target does not actually move in discrete distances, we can smooth this plot by linearly connecting each adjacent change to more accurately represent its motion. 

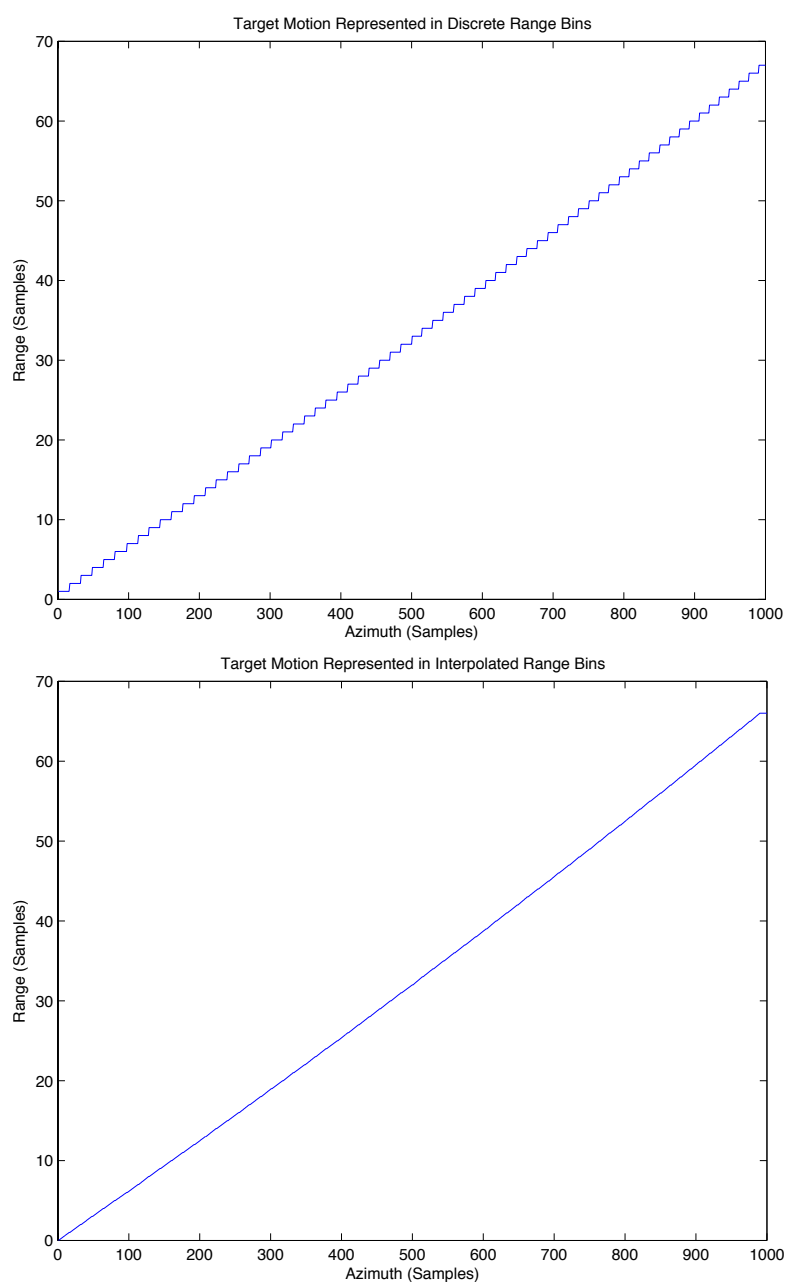

Figure 20: Target distance form the center of the target space in range bins versus slow time samples

Notice in the image to the left we can see that the data appears to be jagged, like a staircase. This is a phenomenon that is a result of working with digital images. Looking at the raw data for this moving target, we cannot see that the data discretely increments towards the right of the image as the target moves along. Once we zoom in on the image to show the motion of the target, we begin to see this pixelation. Artifacts of digital images are unavoidable, but in this case they do not affect the usefulness of the results shown later. 
Once the target's approximate range-bin location is found for each azimuth bin, adjacent approximations can be differenced, giving the sample-to-sample change in range bins for each slow time sample, a first-order discrete time derivative. Using the range bin to distance conversion above, this result converts into distance traveled over azimuth time, or the target's velocity, for each slow time sample.

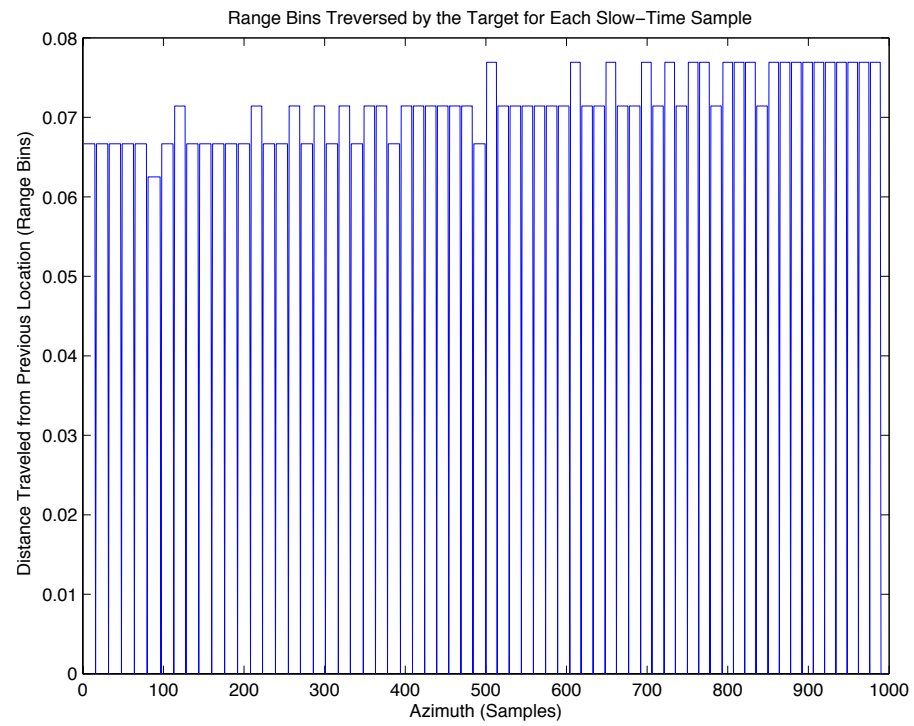

Figure 21: The difference in range bins between each sample

The mean of the data taken in figure 14 gives the target's average velocity over the sampling period. For this particular instance, we can see that the target maintains a somewhat constant velocity. However, for targets moving with nonconstant velocity, this operation can be taken one step further. Starting from the target's range bin velocity, calculating the second-order discrete time derivative gives the target's acceleration in range bins per azimuth bin for each slow time 
sample. The same conversion is used to give the target's approximate acceleration in $\mathrm{m} / \mathrm{s}^{2}$.

An example of this is used for a target accelerating from $0 \mathrm{~m} / \mathrm{s}$ to $50 \mathrm{~m} / \mathrm{s}$ over the simulation duration of 2 seconds, giving an acceleration of $25 \mathrm{~m} / \mathrm{s}$. Following the constant velocity case, the first image is the raw data for the target.

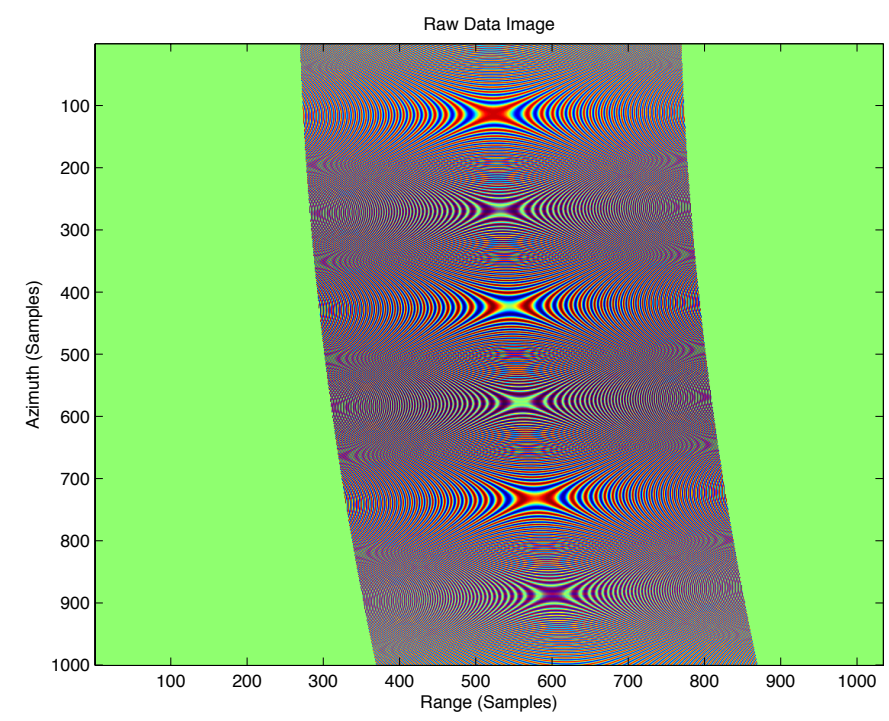

Figure 22: Raw Data for and accelerating target

The data in the image appears curved, because as the target increases in speed, its position and thus its location will increase exponentially. 

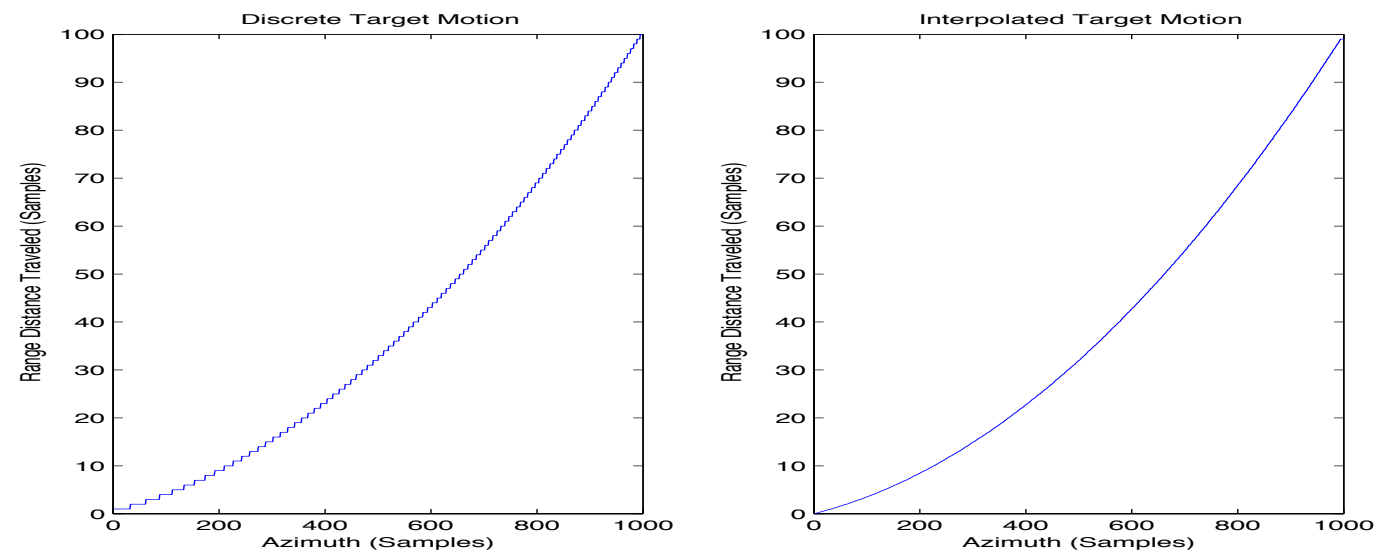

Figure 23: Accelerating Target Location Data

As was done in the constant velocity case, the location of the target for each azimuth sample is found and the trace on the left in figure 21 is plotted; the curve is then smoothed via interpolation, resulting in the image to the right. This image represents an array of data that represents the target's position at any time during sampling. To find its acceleration we need to know how its velocity changes, but the same method mentioned previously for finding velocity cannot be used. Instead, the array representing the targets position is divided element-by-element with the slow time array (STA) which is an array that contains all of the times that the radar system sends out a pulse. Each row in the raw data has a corresponding time value in the slow time array. Dividing the position by the time gives the distance traveled over that time period (i.e. the target's velocity). The result is shown in figure 22. 

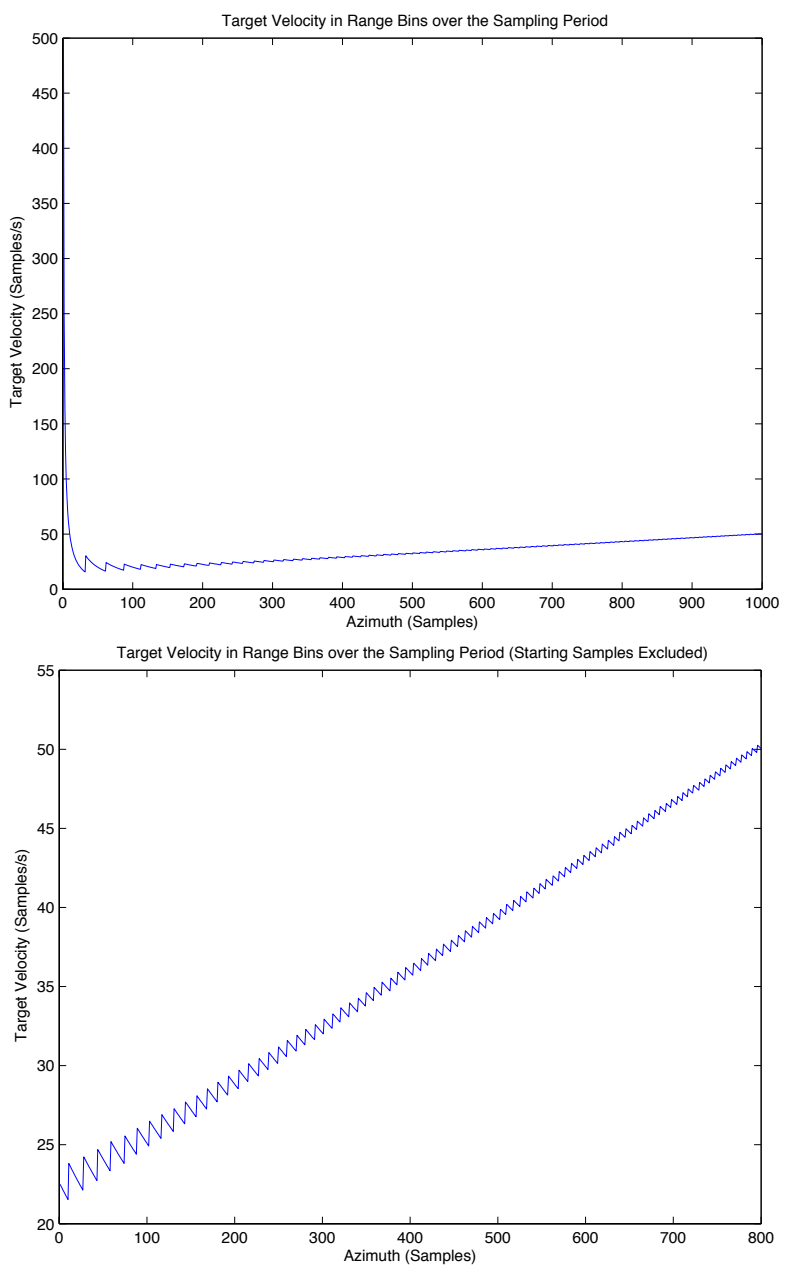

Figure 24: Accelerating target velocity for each slow time sample.

From here, the same approach for finding target velocity in the constant velocity case is taken, except instead of finding the difference in position, it becomes a difference in velocity. Shown here: 


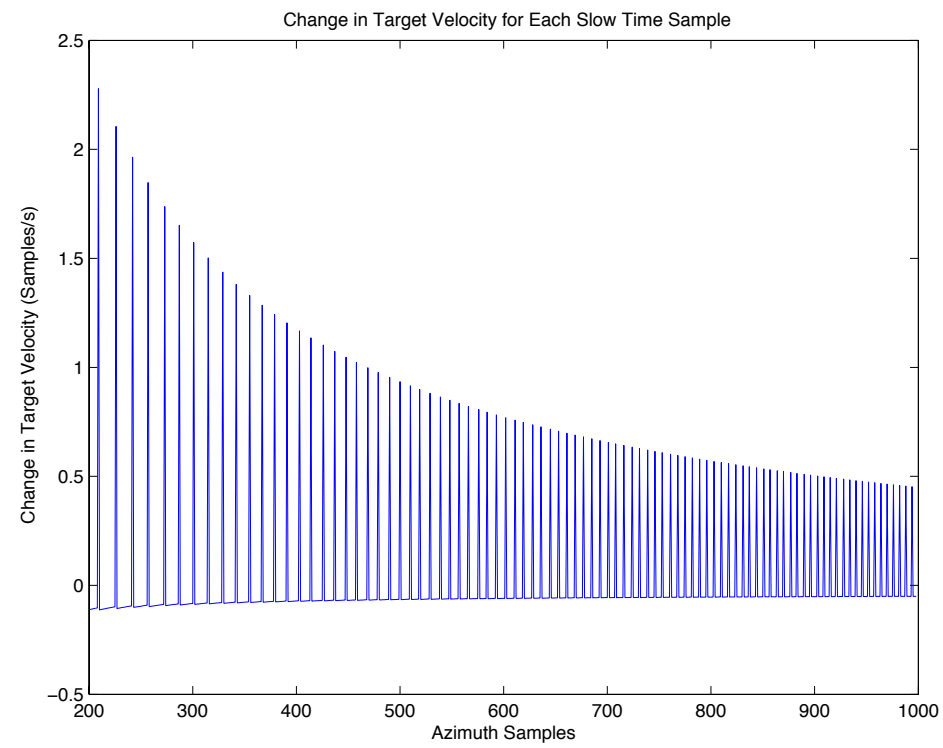

Figure 25: Target acceleration for each slow time sample.

Taking the mean of the data represented in this image gives the target's approximate acceleration for the sampling period in bins $/ \mathrm{s}^{2}$. The same conversion used previously is then used to convert to $\mathrm{m} / \mathrm{s}^{2}$.

\section{Azimuth Direction Motion Detection}

Unlike the in the Range direction, where finding the location of the target was a relatively straightforward task, and the motion data based on those locations was a matter of simply performing time derivatives, the Azimuth direction motion detection is not so intuitive. This section presents a method for detecting azimuth motion with one important assumption1 made. First, it is assumed that the target always starts at the center of the target space and moves strictly in the azimuth direction. Without this prior assumption, the algorithm cannot decide whether the target is moving at a certain velocity, or if it was initially located away from the 
center in the cross-range direction because of this azimuth reference ambiguity. This is not a new concept for this paper. Previous work also relied on this assumption, although the reference to it was not made.

Previous methods for detecting Azimuth motion made use of the trace along the target data line in the range-compressed data. The distance of the peak of this sinc-like waveform from the center of the target space would indicate a constant velocity for the target. Here, the reference point for azimuth motion is also the center of the target space. One thing to note about this method is that it was only useful for calculating an estimated constant velocity. The method presented shows that constant velocities and acceleration can be detected in the azimuth direction.

Furthermore, this method does not follow the order of operations in the traditional Range-Doppler Algorithm. It instead only makes use of small sections of the algorithm, specifically the azimuth fourier transform, matched filter multiplication, and azimuth inverse Fourier transform, and excludes the Range Cell Migration Correction and all other sections of the RDA. For the purpose of selectively performing the individual operations from the RDA, the code from Brian Zaharri's work was split into separate Matlab scripts. Another script was written to run a loop that included all of the relevant operations to gather the data pertinent to the target's motion. 
Starting from the raw data as before, without obtaining the range compressed data, the azimuth Fourier transform is taken, followed by the matched filter multiplication, and azimuth inverse Fourier transform to finish. The mentality behind compressing in the azimuth direction first is because we were able to extract so much range information from the range compressed data without azimuth compression, maybe performing the latter without the former would produce . Here, the range cell migration correction takes much longer to execute because of the extra data, or possibly lack of zero data that exists because the range compression was skipped. RCMC does not affect the outcome of detecting the target's motion with this method, so it was omitted to reduce processing time. The images in figure 26 depict the process for each step mentioned. 

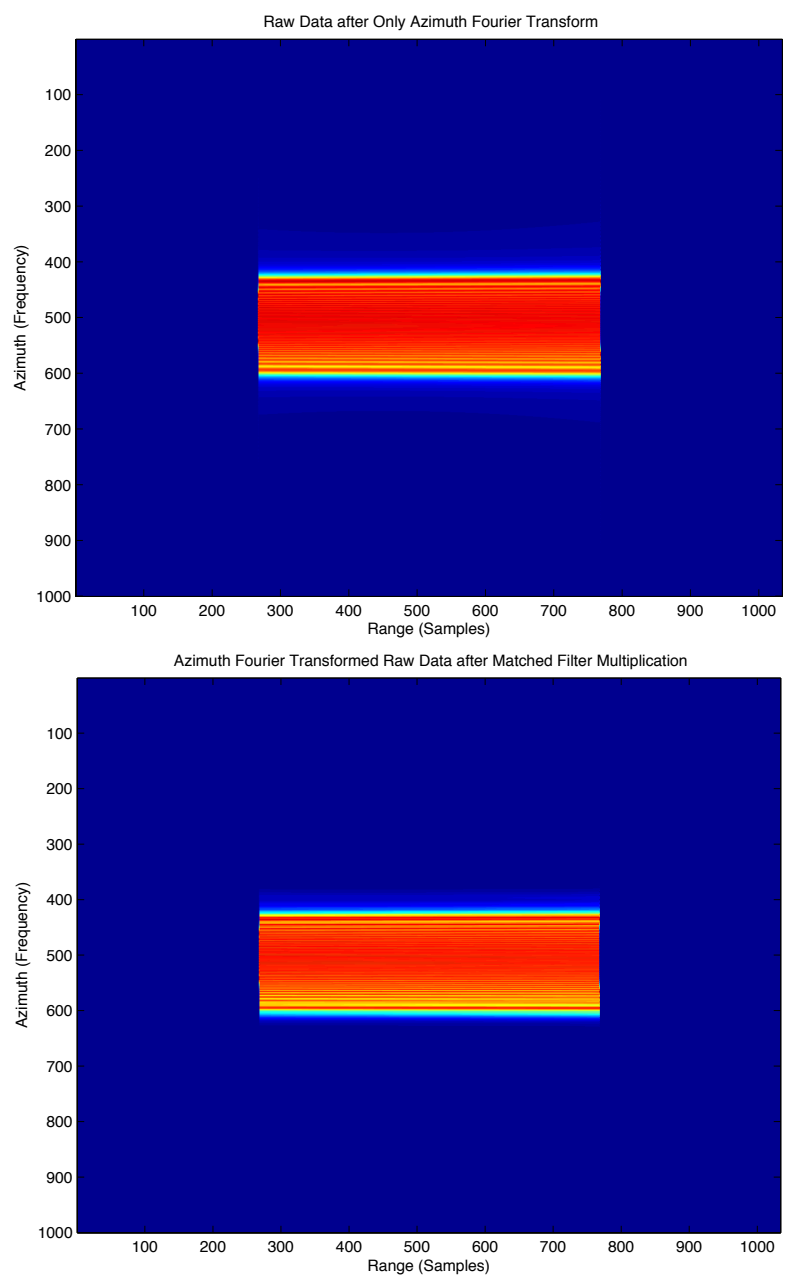

Figure 26: Top-down view of the raw data azimuth Fourier transform of the target. These images can be seen as a top-down view of a height map that shows signal strength.

In this figure we can see the result of the Fourier transform of a target moving in the azimuth direction at $25 \mathrm{~m} / \mathrm{s}$. There is little difference in the appearance between the azimuth transformed data and the matched filtered data. Moving further into the algorithm and plotting the azimuth compressed data gives more information. 

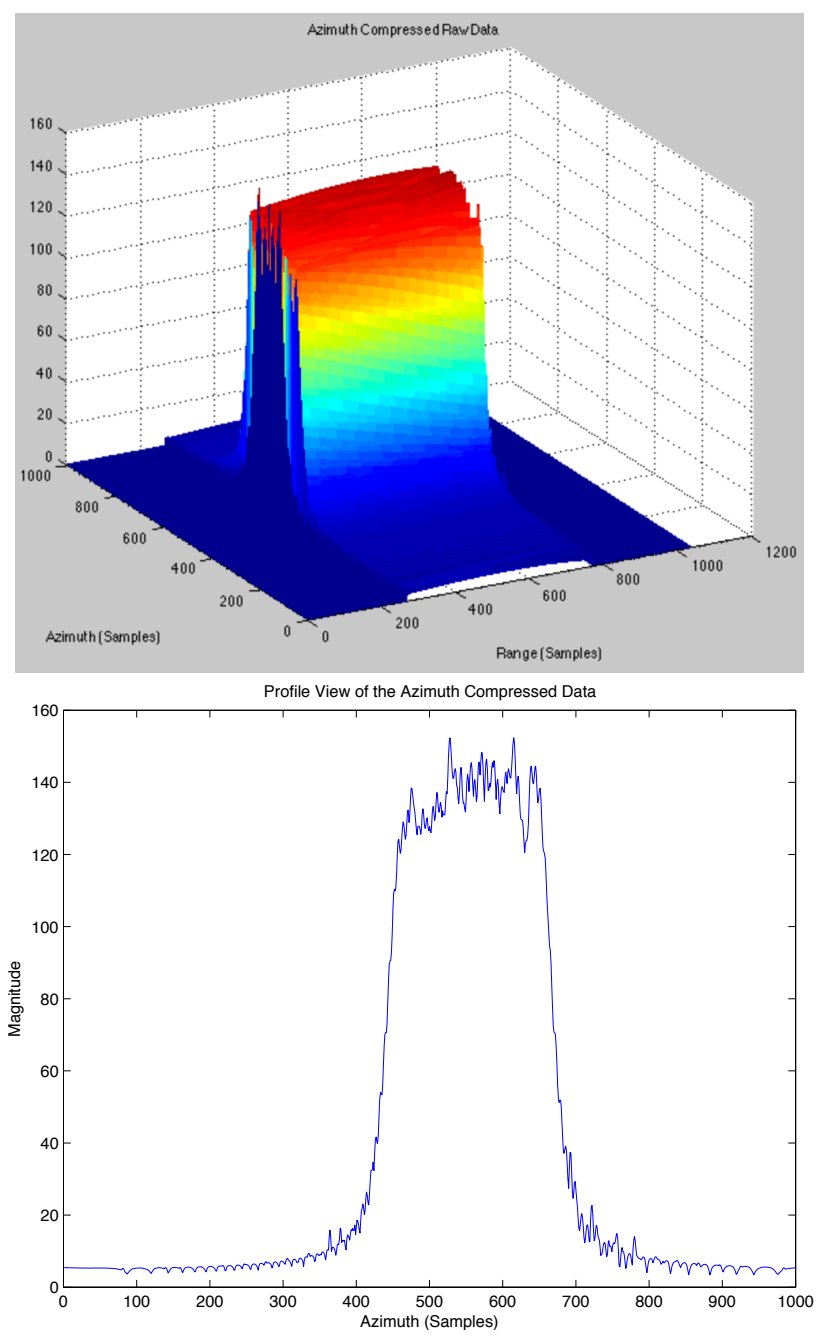

Figure 27: Azimuth Compressed raw data depiction in 3 dimensions to show the magnitude of returns (left), and a profile view of this data looking down range (right)

Here we see the result of azimuth compression. The profile view of this data looking down range (on the right) can be called the Azimuth Compressed Raw Data Profile, or ACP for short. The ACP contains several usable pieces of data for calculating the target's motion information. As to why the ACP appears so jagged might be contributed to the missing steps in the range direction, or the lack of 
compensation code present in previous works. Explaining and correcting this may improve the effectiveness of this method greatly and is to be explored by future research. Consider the following two ACP plots and compare them to the $25 \mathrm{~m} / \mathrm{s}$ target's ACP.
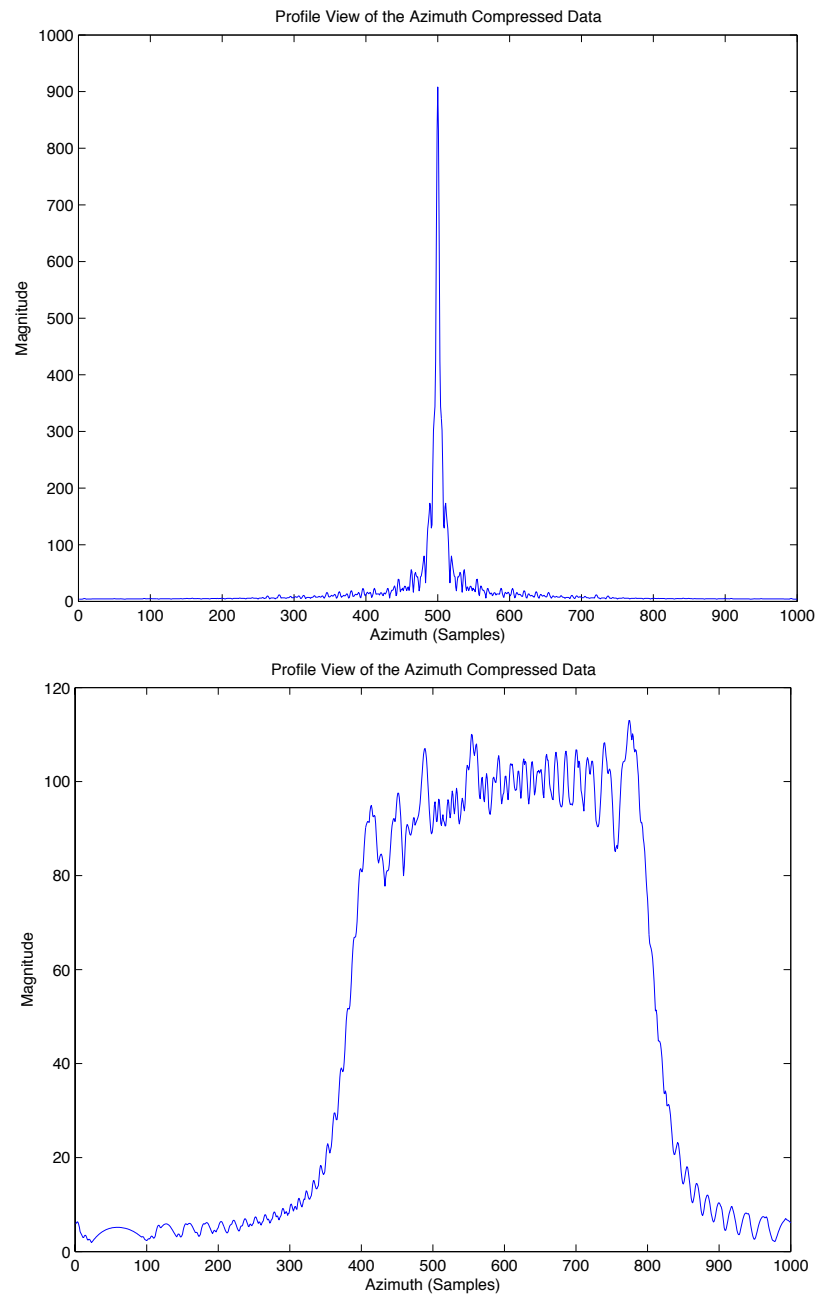

Figure 28: Azimuth compression data down-range profile view for targets traveling at $0 \mathrm{~m} / \mathrm{s}$ (left) and $50 \mathrm{~m} / \mathrm{s}$ (right). 
The three plots show targets traveling at $0 \mathrm{~m} / \mathrm{s}, 25 \mathrm{~m} / \mathrm{s}$ and $50 \mathrm{~m} / \mathrm{s}$. From these plots, four metrics can be observed: the maximum height, the mean of the cluster, the width, and the azimuth bin estimate or the approximate center location of the return cluster's density along the azimuth. The maximum height and average height for the ACP is found by using the Matlab $\max ()$ and mean() functions, which respectively return the maximum value and mean value in a vector; The width was found by collecting the number of azimuth bins in the ACP with magnitudes greater than ten percent of the maximum height; the azimuth bin estimate was found by summing the individual azimuth bin signal magnitudes until half of the total signal magnitude for all azimuth bins was reached. 


\section{IV.SIMULATION RESULTS AND ANALYSIS}

To evaluate the methods described, different analysis methods were employed for each direction. The range analysis was relatively straightforward and required the collection of a single set of motion data for constant target velocities and accelerations. For the range, constant position analysis for this algorithm would have been a trivial task. The output would be a completely straight set of raw data that would shift with each iteration. Because of this fact, constant position was skipped for the range direction. The azimuth data was not so simple, as it required that the ACP data be collected prior to the analysis. In order to perform these tests, a looping script was used that makes use of the sub blocks mentioned for each direction. Using this loop with the specified sub algorithms reduced the amount of time required to run the entire test significantly when compared to the original code by a ratio of 10:1 for the range direction and 4:1 for the azimuth direction.

\section{Range Direction Results}

\section{Data Set 1: Constant Velocity Targets}

To analyze the accuracy of the range motion detection, two tests were run: one for constant velocities, and one for constant accelerations. For each iteration, the algorithm would estimate the type of motion that the target was experiencing, stationary, constant velocity, or accelerating, and give a percent error based on the real input and the estimate:

$$
\text { Percent Error }=\frac{\text { Actual Paramter }- \text { Estimated Parameter }}{\text { Actual Parameter }} * 100 \%
$$


This equation is used to calculate percent error for all of the percent error plots in this paper. The following images show the data for targets moving

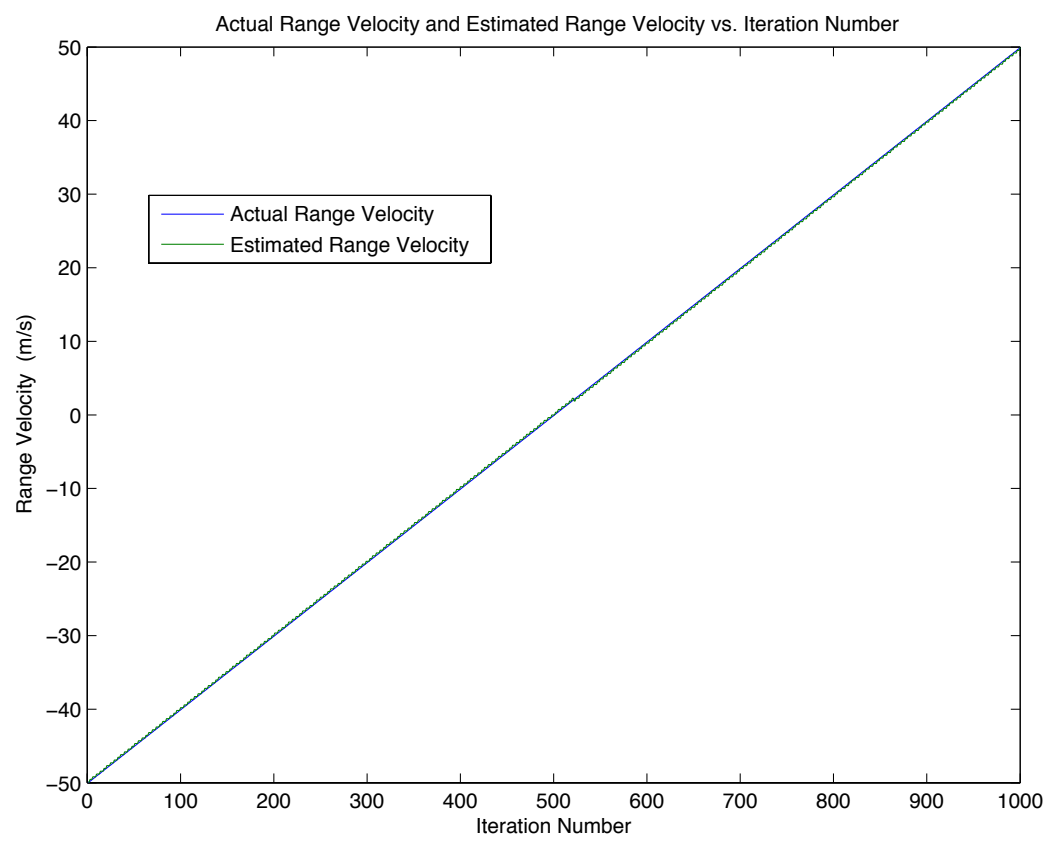

\section{Figure 29: Actual Target Velocity and Estimated Target Velocity for 1000 iterations.}

This image shows the actual target velocity plotted with the target velocity estimates made by the algorithm. Because the raw data so blatantly shows the position for each sample, the error in estimating the position along the range is relatively trivial, and calculating the velocity is a matter of taking a discrete time derivative of very accurate data: 

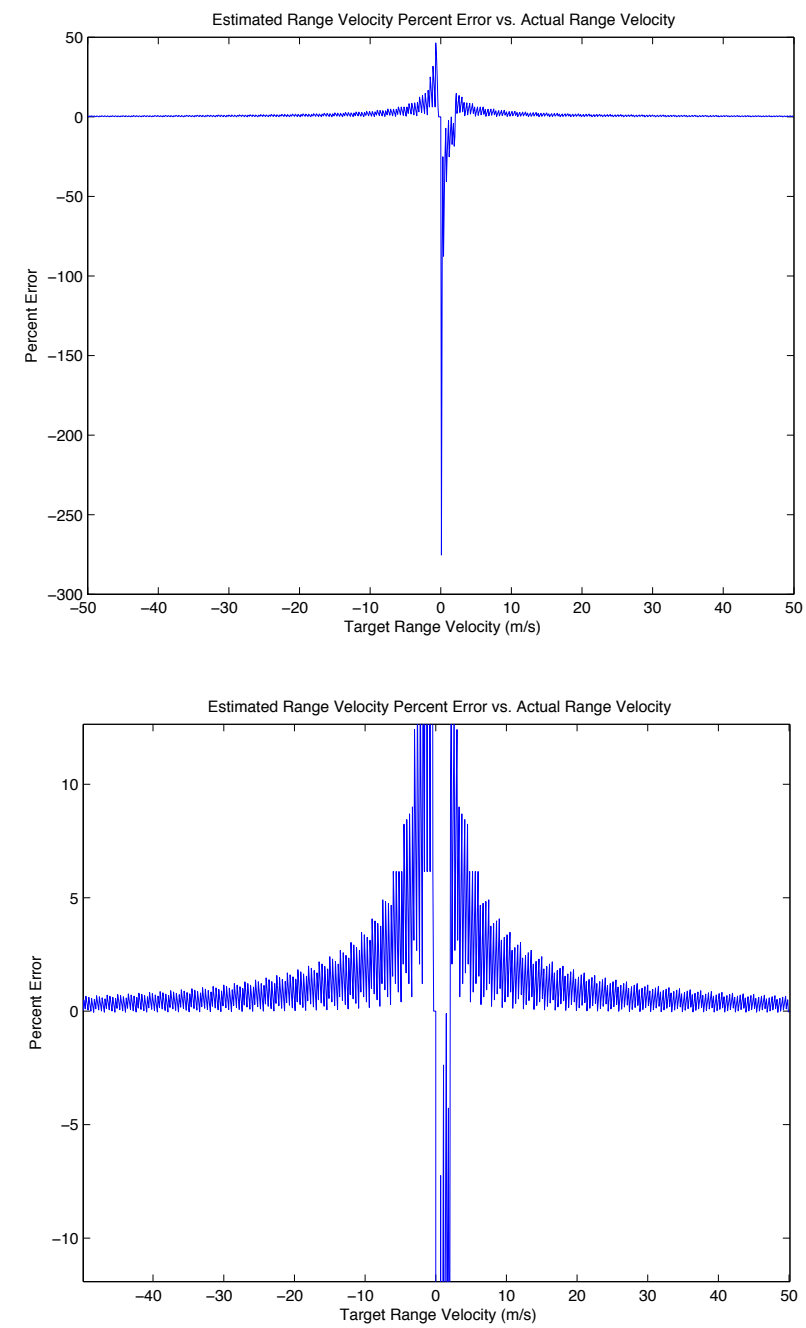

Figure 30: Percent error in estimating target's range velocity

We can see that the error in estimating is small, other than those centered around zero. This is because the data used for comparison is discrete and so are the data points used for the simulation. As the actual velocity approaches zero, the imperfections of the algorithm show by having a minimum error dictated by the resolution of the data. A estimate made by the algorithm that is a few tenths of a meters per second off when the actual velocity is less than $0.1 \mathrm{~m} / \mathrm{s}$ would result in a percent error that is hundred of percentage points off. Although percent error is 
an important metric, the absolute error for each measurement is shown alongside it, to show that the error present around zero m/s does not actually have much of an impact on the estimates.

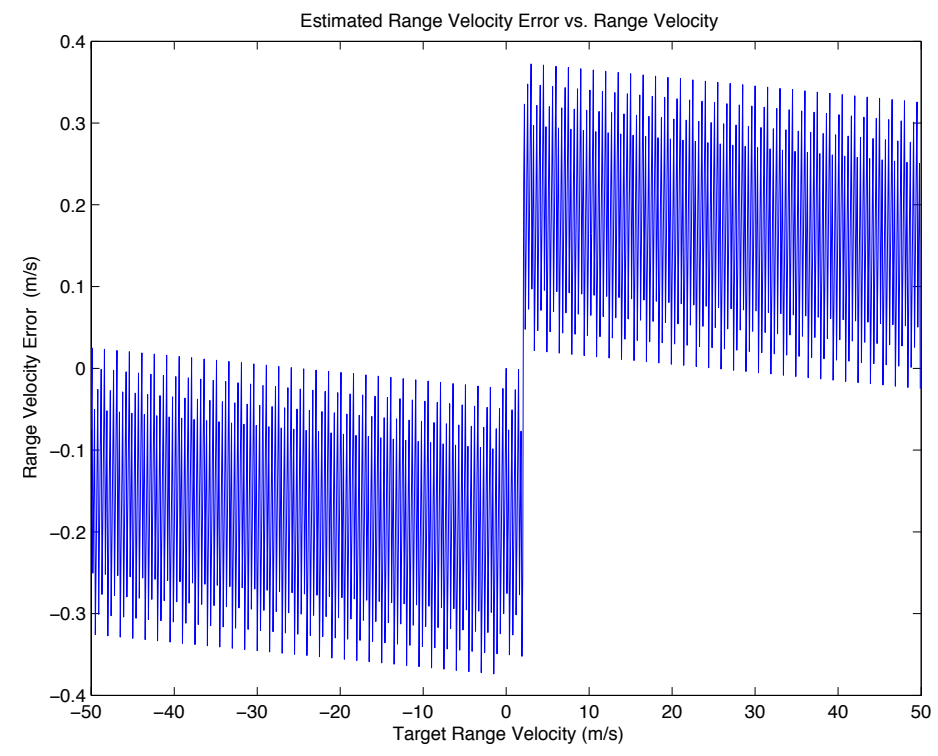

Figure 31: Velocity error in estimating the target's range velocity.

This data shows that the algorithm is always within $0.4 \mathrm{~m} / \mathrm{s}$ from the actual target's range velocity. Even though the percent error shown in the previous plot was in the hundreds of percent, this plot shows that it does not impact estimating as much as it appears to. The average percent error excluding those center values is $0.962 \%$ with a variance of $1.025 \%$.

\section{Data Set 2: Accelerating Targets}

This data shows a target starting from a stationary state and accelerating. The acceleration is constant for each iteration and the maximum velocity ranges from - 
$50 \mathrm{~m} / \mathrm{s}$ to $50 \mathrm{~m} / \mathrm{s}$. Since the simulation represents two seconds worth of data, this translates to constant accelerations of -25 to $25 \mathrm{~m} / \mathrm{s}^{2}$.

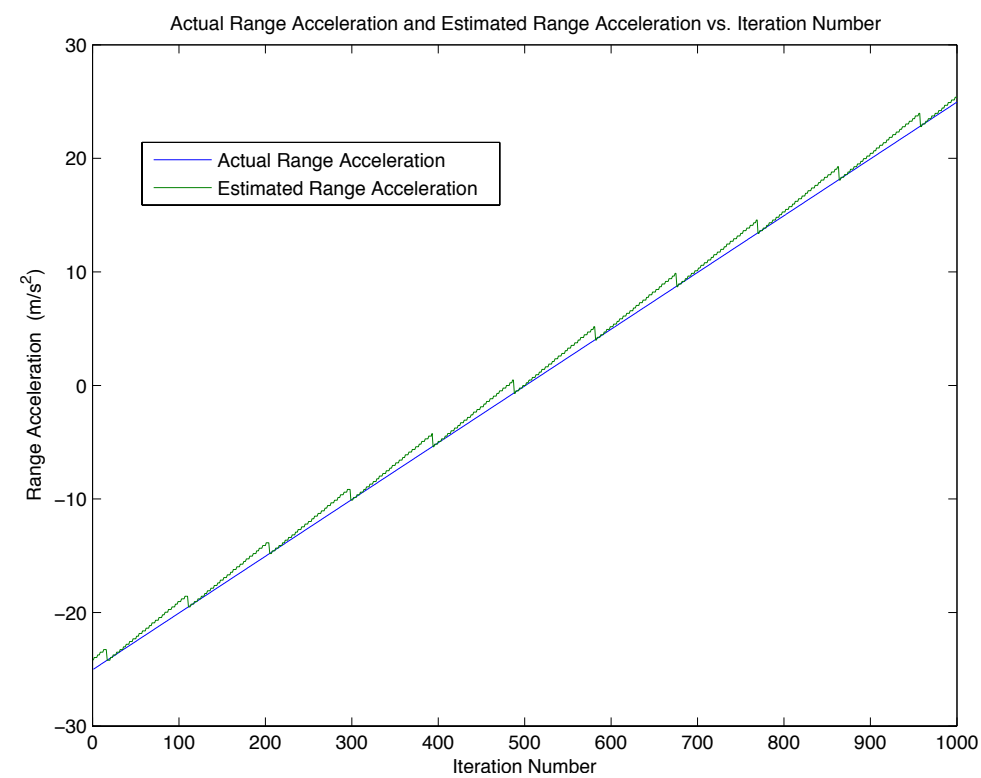

Figure 32: Target actual range velocity plotted against the range velocity estimated by the algorithm

The estimates seem to increase in error as the target's acceleration becomes more positive, but then they correct themselves, creating a sawtooth effect on the estimates. This shows up in the percent error. 


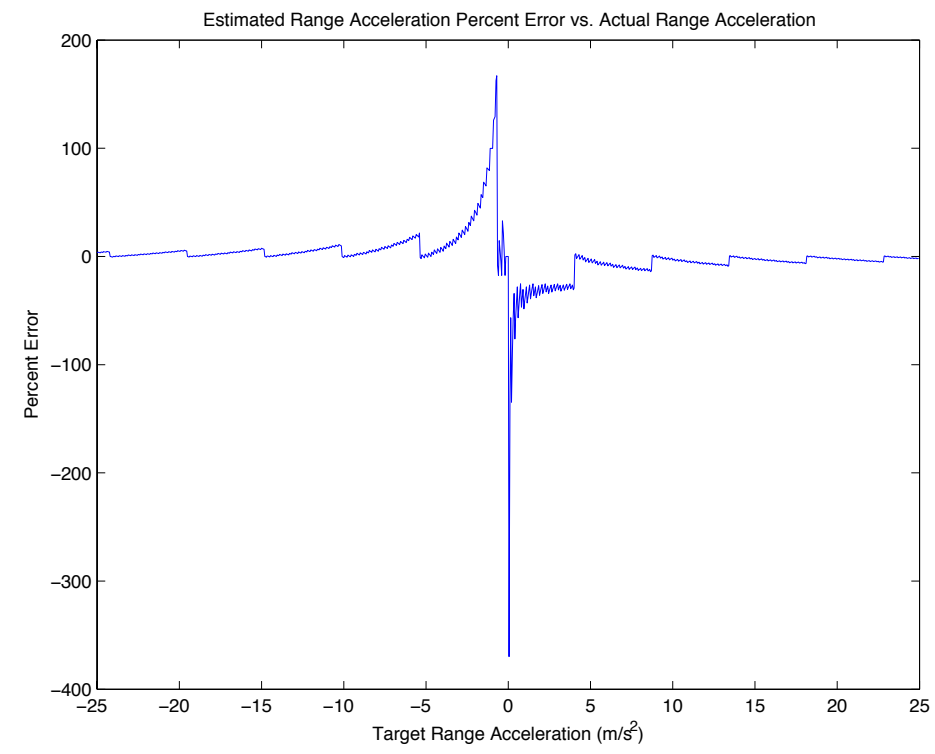

Figure 33: Percent error when estimating the target's range acceleration.

The sawtooth effect leaks over into the error plot, but the error seems to be within a $1 \%$ margin. In fact, excluding the asymptotic data in the center as before, the mean percent error is $-0.4 \%$, with a variance of $67 \%$. Because the estimates are for the most part more positive than the actual acceleration values, as the actual acceleration approaches zero, the percent error increases as mentioned before. This is why the variance appears to be so high. For the data included, the percent error is as much as $27 \%$. However, the higher percent error values such as this one are located around the smaller values of acceleration. The percent error seems to trail off with higher acceleration values. 


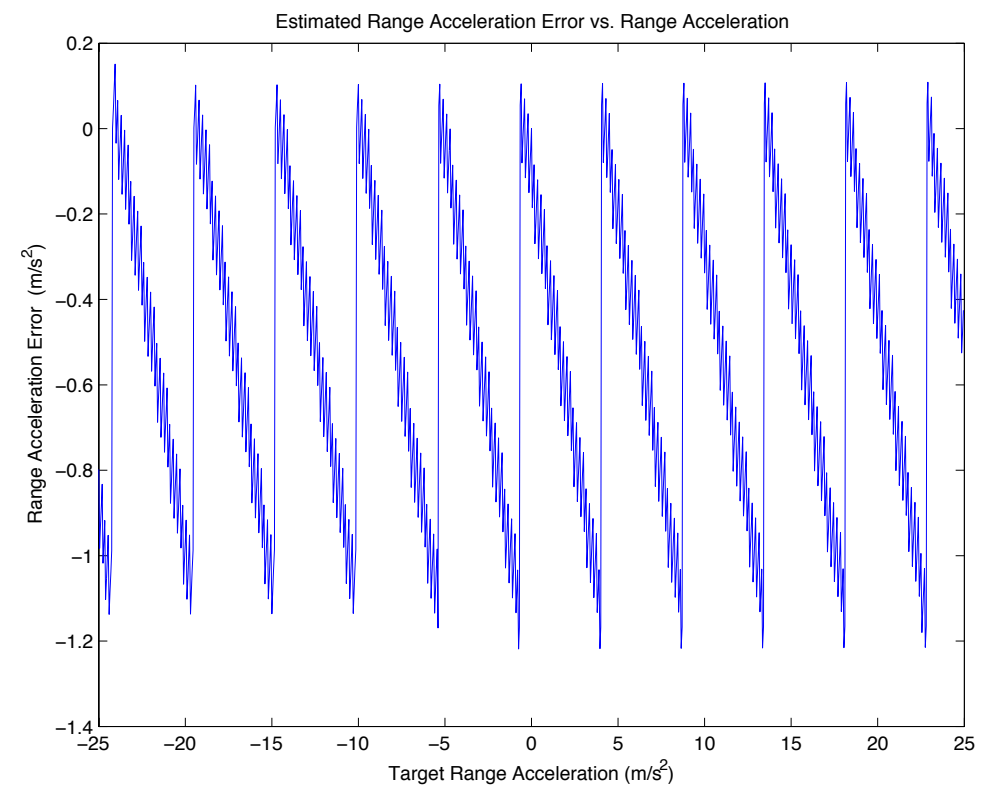

Figure 34: Acceleration error when estimating the target's range acceleration.

The error stays within a constant range throughout the sampling. Looking at the absolute acceleration estimating error above, although the variance was very high for the percent error, we can see that the absolute error has a maximum absolute value of $-1.2 \mathrm{~m} / \mathrm{s}^{2}$. The Absolute errors all appear to be negative because of the (Actual Parameter - Estimated Parameter) term. It is not apparent why the algorithm consistently estimates higher than the actual acceleration values, but will be discussed in the future work section.

\section{Azimuth Direction Data Collection}

To determine the effect that target velocity has on each of the metrics mentioned at the end of the previous chapter, the algorithm was run and data collected on one thousand iterations for each type of condition: constant target positions ranging 
from -190 meters to +190 meters from the target space's center along the azimuth direction; constant target velocities ranging from $-50 \mathrm{~m} / \mathrm{s}$ to $+50 \mathrm{~m} / \mathrm{s}$; and a third test with constant accelerations ranging from $-25 \mathrm{~m} / \mathrm{s}^{2}$ to $+25 \mathrm{~m} / \mathrm{s}^{2}$.

\section{Data Set 1: Stationary Targets}
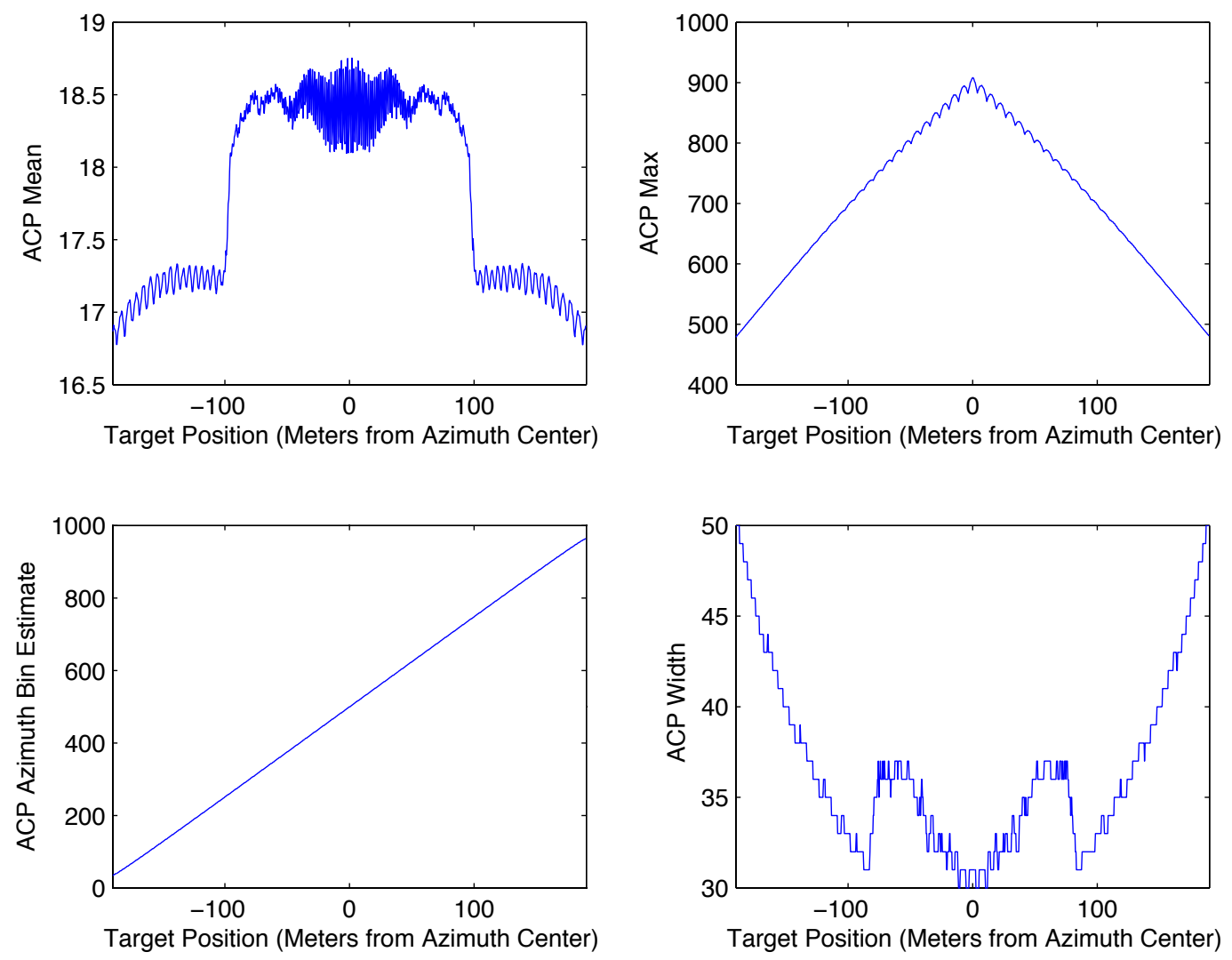

Figure 35: ACP Maximum, Mean, Width, and Azimuth Bin Estimate for a stationary target with each iteration incrementing the position from-190 meters to 190 meters from the center of the azimuth in increments of 0.038 meters.

We can comment on several things from the data plotted. First, the mean is not considerably affected by location on the azimuth axis for stationary targets. 
Second, the maximum and azimuth bin estimates both change linearly with position. The width data has extra artifacts, which we see as the two lumps centered around the origin, that make the data 2 to 1 for some width values. To fix this issue, widths less than 70 bins, which corresponds to targets traveling less than $3.5 \mathrm{~m} / \mathrm{s}$, are considered to be stationary targets automatically. We will see later that this setting of 70 bins does not affect the velocity and acceleration data sets, as their bin widths are greater than 70 for velocities greater than a few meters per second. It also allows us to ignore data that acts asymptotically 


\section{Data Set 2: Constant Velocity Targets}
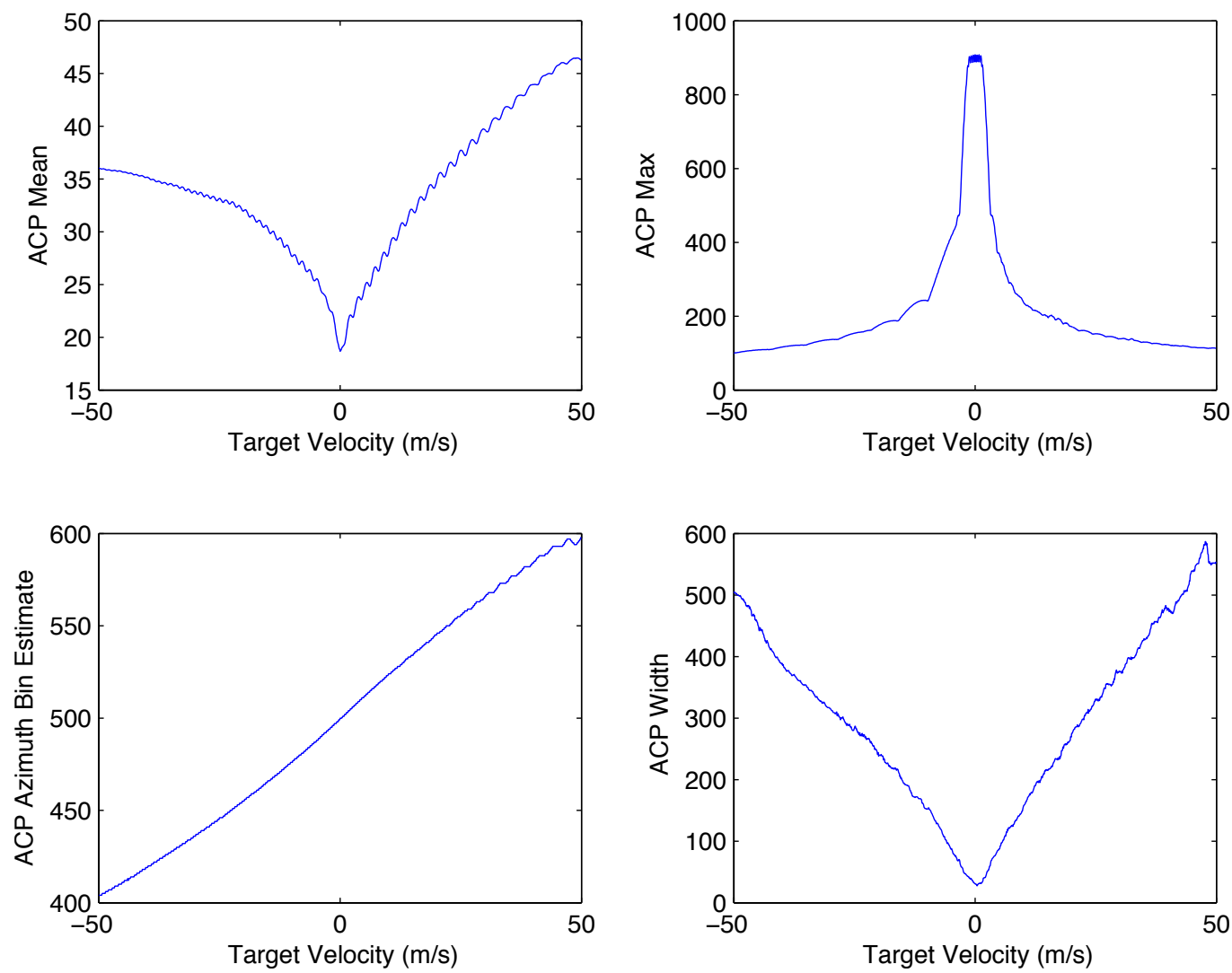

Figure 36: ACP Maximum, Mean, Width, and Azimuth Bin Estimate for target with each iteration incrementing the velocity from $-50 \mathrm{~m} / \mathrm{s}$ to $50 \mathrm{~m} / \mathrm{s}$ in increments of $0.1 \mathrm{~m} / \mathrm{s}$.

Here we see the plotted ACP metric values for constant target velocities ranging from $-50 \mathrm{~m} / \mathrm{s}$ to $50 \mathrm{~m} / \mathrm{s}$. As was seen with the ACP plots for the target moving at 0,25 and $50 \mathrm{~m} / \mathrm{s}$, as the absolute value of the target's velocity increases, the ACP's maximum decreases, while the width and the targets absolute bin estimate from the center value both increase. What was difficult to see before was that the mean of the ACP data also increases as its absolute velocity increases. Notice how the mean values increase a different rates for positive and negative velocities. It is 
suspected that this occurrence is due in part to the geometry of the simulation, much like range cell migration and has the possibility of being corrected; however, this paper does not present an approach to do so and rather uses this circumstance in determining whether the target is moving positively or negatively with respect to the center of the target space. Also note that the minimum bin width limit of 70 does not affect this data set. Looking at the widths of target ACP data from the bottom right plot of the figure above, we can see that just a few meters per second translates to bin widths above the 70 bin minimum set earlier. 


\section{Data Set 3: Linearly Accelerating Targets}
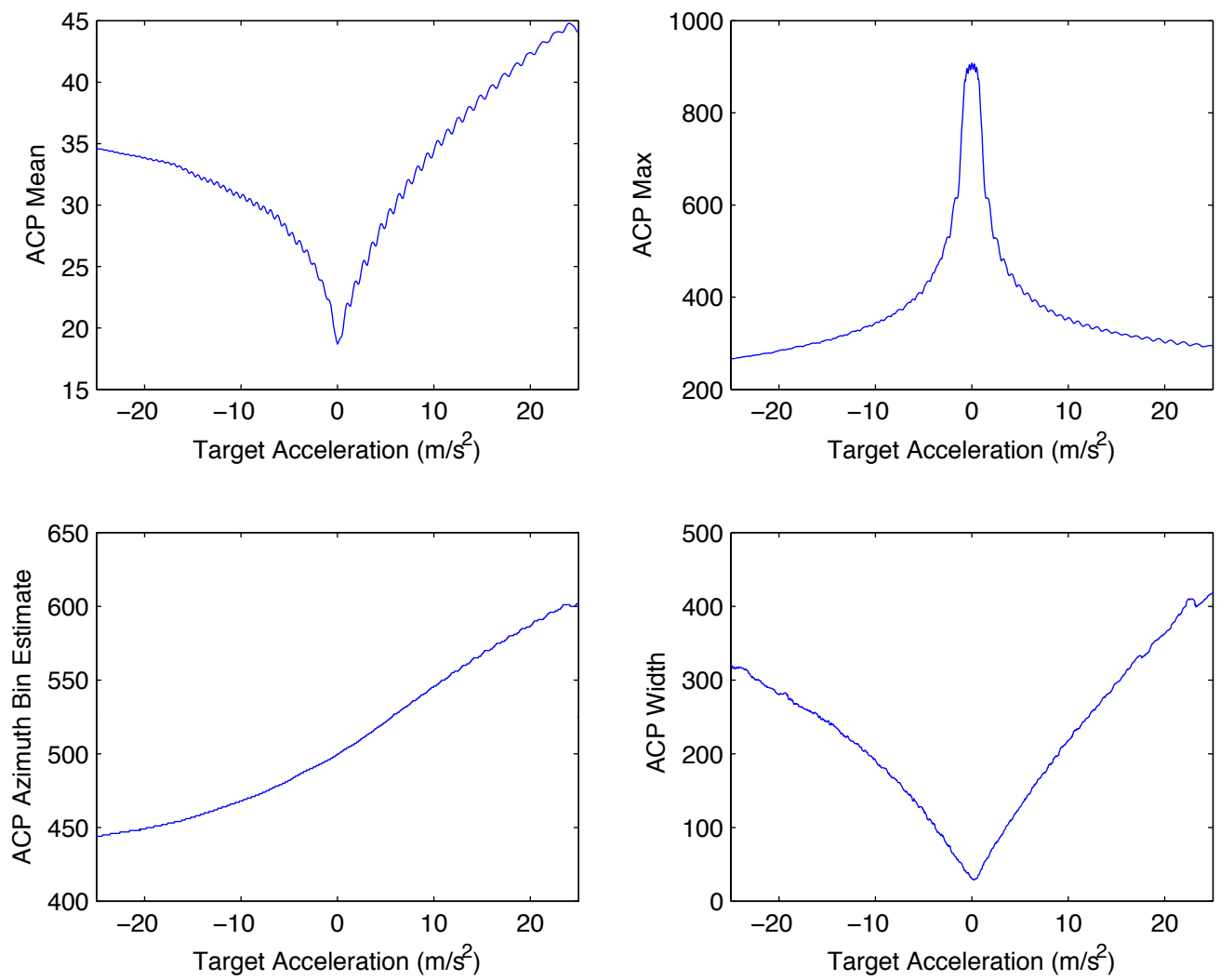

Figure 37: ACP Maximum, Mean, Width, and Azimuth Bin Estimate for target with each iteration incrementing the acceleration from $-25 \mathrm{~m} / \mathrm{s}^{2}$ to 25 $\mathrm{m} / \mathrm{s}^{2}$ in increments of $0.05 \mathrm{~m} / \mathrm{s}^{2}$.

These plots show the ACP data for constant target accelerations ranging from -25 $\mathrm{m} / \mathrm{s}^{2}$ to $25 \mathrm{~m} / \mathrm{s}^{2}$. For this set, the target starts at a stationary position and accelerates in either the positive or negative direction. The plots look very similar to the velocity data, but with considerable differences in the values. Note that for all of these cases, the duration of the simulation is two seconds, meaning that the target reaches the velocities shown in the velocity figure by the end of the sampling period. 


\section{Azimuth Motion Detection Results}

The data plotted in the three previous figures shown is used as a set of look up tables to compare target ACP data with during testing. To do this, the target's ACP data is extracted from the simulation results, and then compared to all the values in each of the data sets. While there are some 2-to-1 mean, width, and max values for many of the data points in all plots, because they all vary across constant position, velocity and acceleration values, and then between positive and negative motion, the least squared error method seems to be the simplest and most accurate decision making method for this case. 


\section{Data Set 1: Stationary Targets}

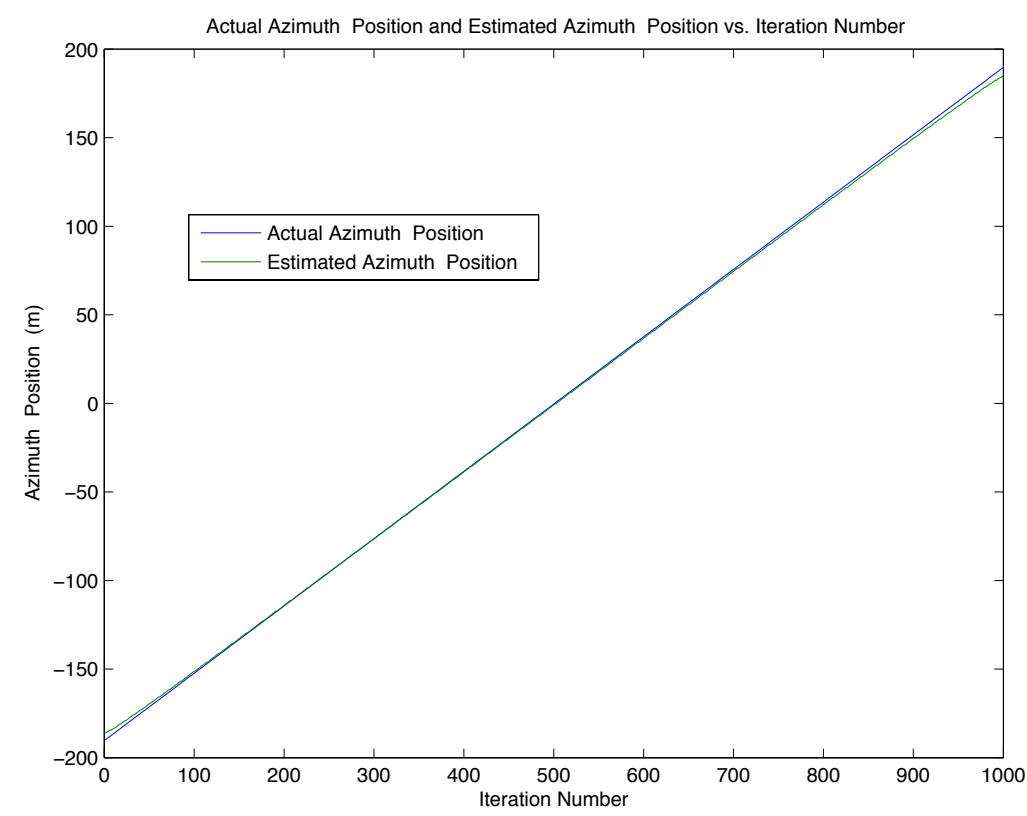

Figure 38: Actual target position and estimated target position for 1000 iterations.

This image shows the actual target azimuth position plotted with the target azimuth position estimated by the algorithm. The actual target position, plotted in blue, was ranged from $-190 \mathrm{~m}$ to $190 \mathrm{~m}$ in 1,000 iterations. The estimates made by the algorithm, plotted in green, almost completely overlap the actual positions of the target. The next plot shows the percent error for each estimate. 


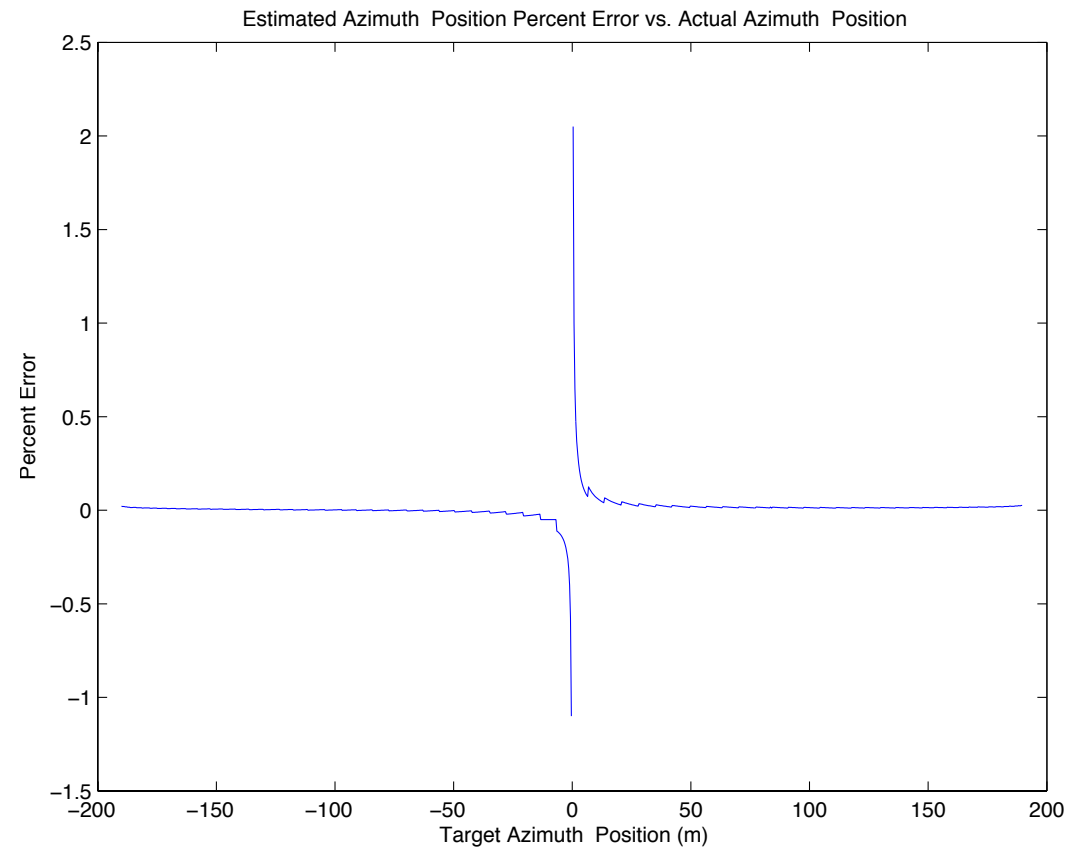

Figure 39: The percent error from estimating Target Azimuth Position.

The first thing to notice about this plot is the asymptotic behavior at the origin. Because the actual position is varied linearly from negative values to positive values, it will cross very closely to zero such that the estimates can be mere tenths of a meter, more or less, than the actual value and have a percent error in the hundreds of percentage points. This does not occur when the actual value is further from zero. Excluding the asymptotic data in the center of the plot marked by the two highlighted data points, the mean percent error is $0.89 \%$, with a variance of $0.01 \%$. This translates to the following plot, which shows the algorithm's error in meters. 


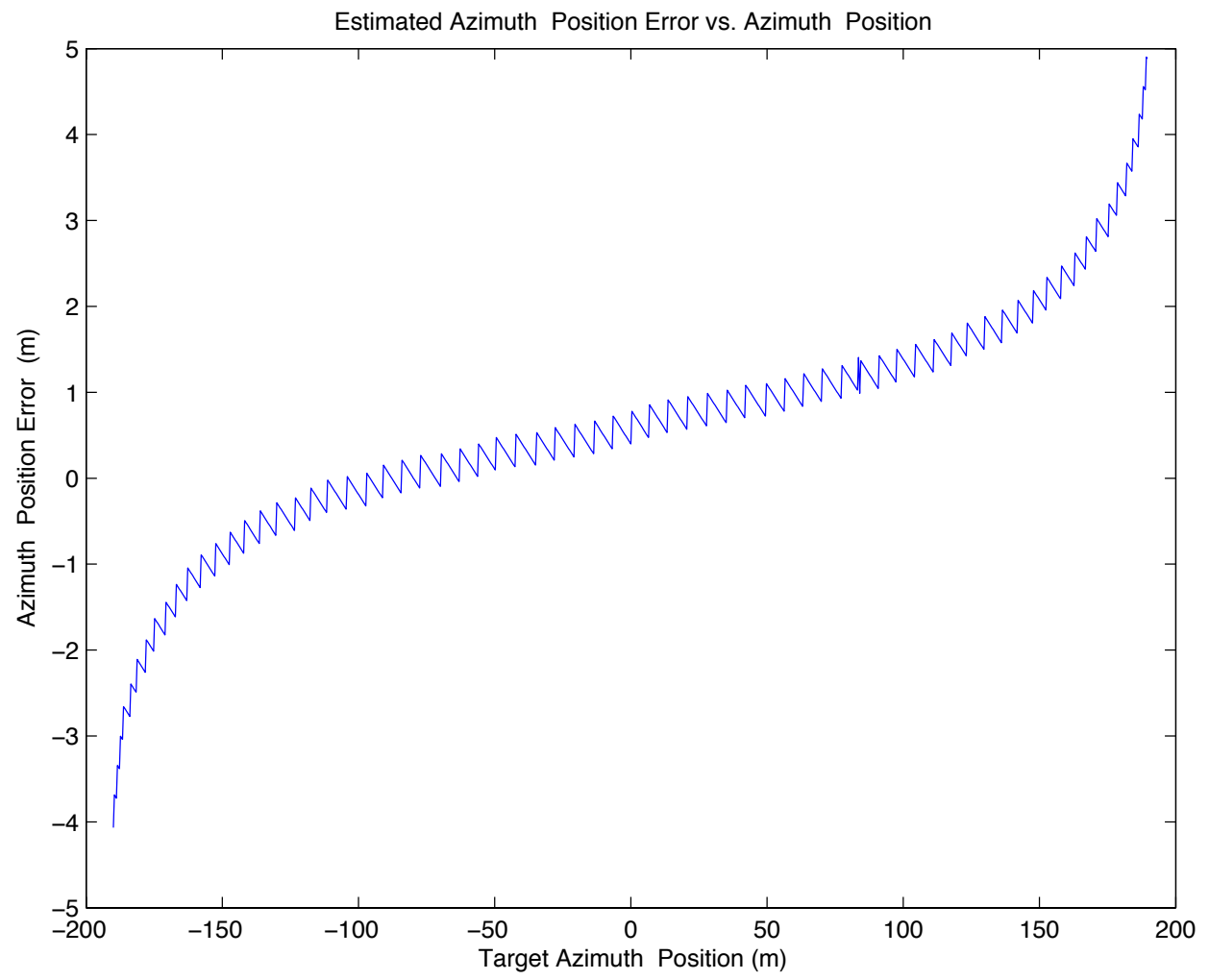

Figure 40: The error in meters, estimating Target Azimuth Positions.

This plot simply shows the difference between the actual azimuth position and the estimated azimuth position. The algorithm does not estimate more than 4 meters away from the target's actual position, which equates to roughly $1 \%$ of the entire target space's azimuth width. Furthermore, the plot shows that the estimating error follows a third order exponential curve with a single zero at the origin. This may be accounted for to improve the algorithm's position estimating accuracy. Further discussion can be found in the future work section. 


\section{Data Set 2: Constant Velocity Targets}

The following three plots show the results for moving targets with constant velocities ranging from $-50 \mathrm{~m} / \mathrm{s}$ to $50 \mathrm{~m} / \mathrm{s}$.

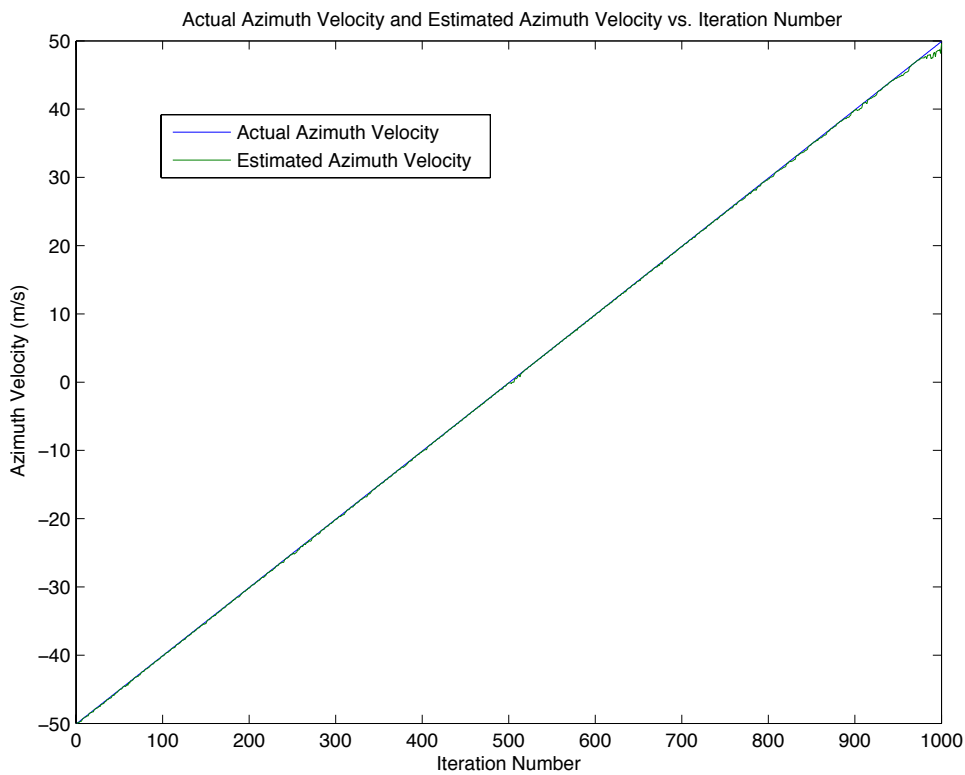

Figure 41: Actual Azimuth Velocity and Estimated Velocity vs. Iteration Number

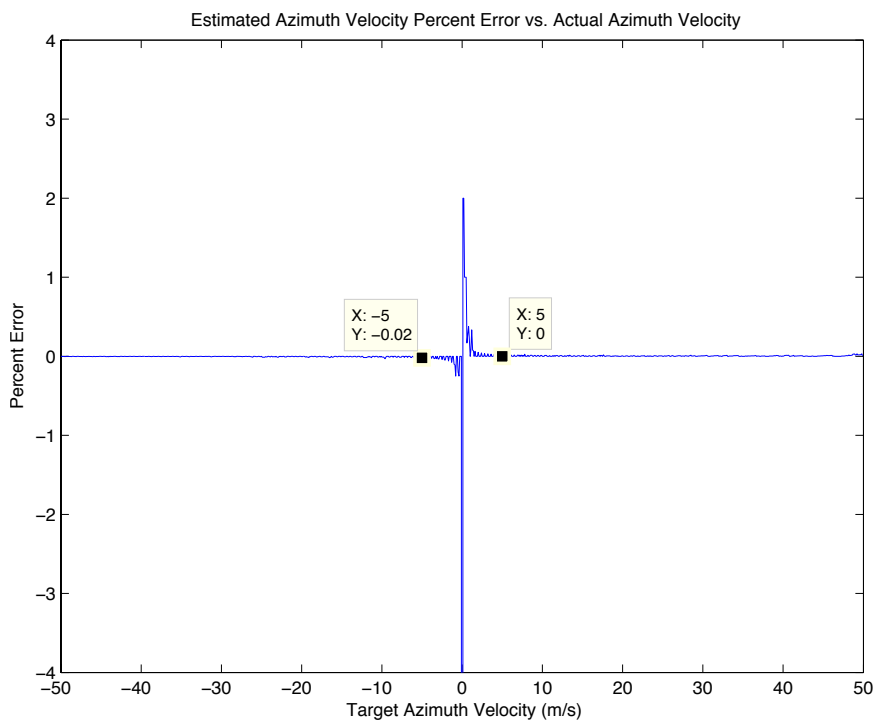

Figure 42: Estimated Azimuth velocity percent error. 


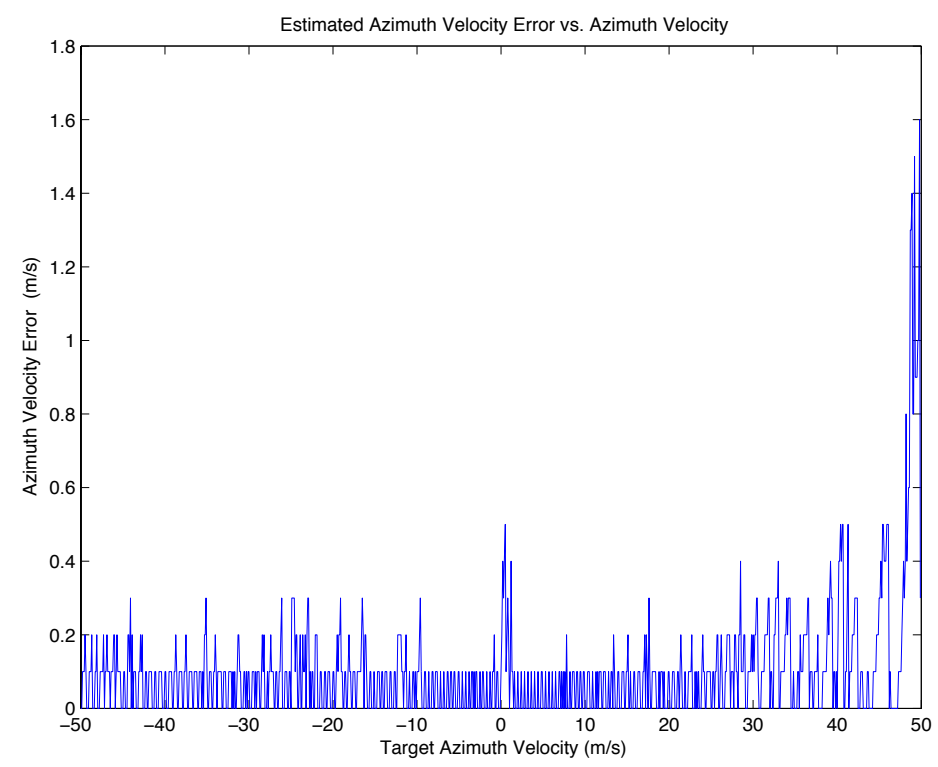

Figure 43: Estimated Azimuth Velocity Error vs. Azimuth Velocity

In the first image, the estimated azimuth position values very closely overlap the actual azimuth position values for every sample. We can see the accuracy of these estimates from the percent error plot from figure 39. To avoid distortion caused by the slow motion data's asymptotic values, 100 samples were removed from the center of the plot to avoid including the asymptotic data. This data is characterized by a mean percent error of $0.4016 \%$ with error variance $0.402 \%$. According to this data, the algorithm estimates the velocity to be more positive than the actual velocity. Since the estimates are based on the previously collected motion data, a suspect for this occurrence could be the high frequency content present in the accelerating target ACP data. Further discussion can be found in the future work section. The final plot shows the actual error in meters per second. Generally speaking, the algorithm can estimate a target's velocity to within 1.6 
meters per second for all tested velocities. The higher valued positive velocities tend to be slightly harder for the algorithm to estimate because that is where the largest error seems to be located, but with the exception of $48-50 \mathrm{~m} / \mathrm{s}$ targets, the algorithm can estimate to within $1 \mathrm{~m} / \mathrm{s}$.

\section{Data Set 3: Accelerating Targets}

For this set, the target begins in a stationary state at the center of the target space and accelerates in the positive or negative direction. The accelerations are constant for each iteration and range from $-25 \mathrm{~m} / \mathrm{s}$ to $25 \mathrm{~m} / \mathrm{s}$.

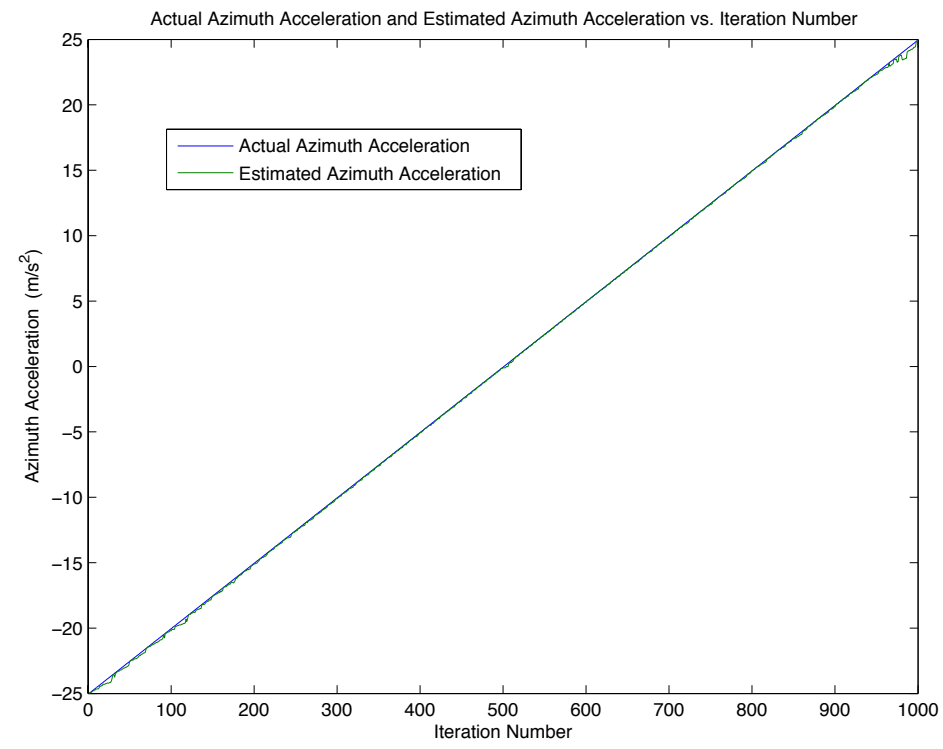

Figure 44: Actual Azimuth Acceleration and Estimated Azimuth Acceleration v. Iteration Number

As is the trend, we see very close overlapping with the actual and estimated values for acceleration. We can thusly expect small percent error values. 


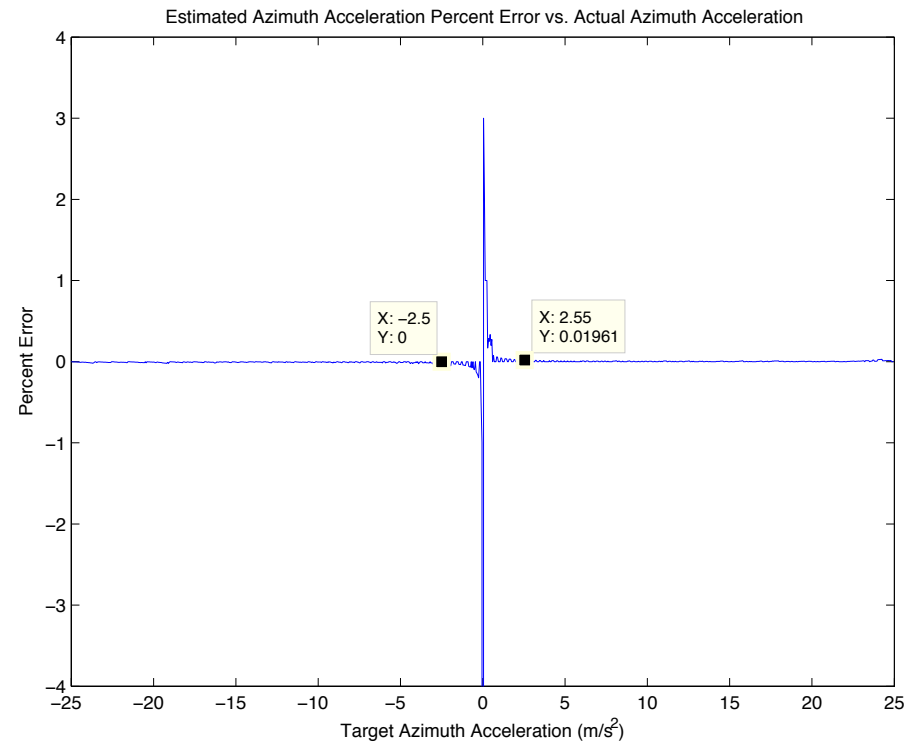

Figure 45: Estimated Azimuth Acceleration Percent Error v. Azimuth

\section{Acceleration}

The percent error, with the exclusion of the 100 points located around $0 \mathrm{~m} / \mathrm{s}^{2}$, has a mean value of $0.145 \%$, with a variance of $0.563 \%$.

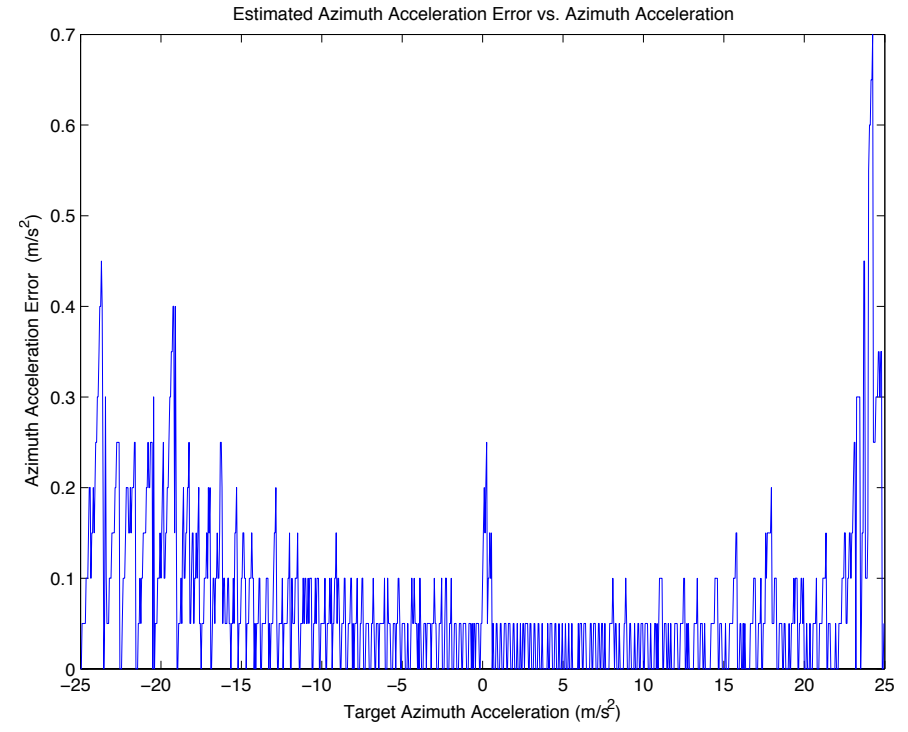

Figure 46: Azimuth Acceleration Error v. Azimuth Acceleration 
The maximum acceleration error from all of the estimates is $0.7 \mathrm{~m} / \mathrm{s}^{2}$. The algorithm can comfortably estimate within $0.7 \mathrm{~m} / \mathrm{s}^{2}$, but this point is an outlier, and the algorithm can estimate to within $0.5 \mathrm{~m} / \mathrm{s}^{2}$ for most target acceleration values. The error values are discrete in appearance because both the actual and estimated acceleration values increment by $0.5 \mathrm{~m} / \mathrm{s}^{2}$, therefore the errors will all be in multiples of $0.5 \mathrm{~m} / \mathrm{s}^{2}$. This outcome also appears in the velocity estimate error, but it does not hinder the data's ability to show that velocity and acceleration can be approximated with this algorithm. It does provide some insight into how a finer increment would reduce the appearance of the discrete nature of the data, although this would require that the data sets used for comparison have far more data points with which to compare. While this would increase the quality of the data by reducing the amount of error, processing time would increase because more comparisons would need to execute. Here we see the tradeoff between resolution and accuracy, but since we are already within a $1 \%$ error margin, increasing the resolution of the captured motion data would not add significant value to the test. 


\section{CONLUSION AND SCOPE FOR FUTURE WORK}

\section{Concluding Statements}

In this paper, it was argued that rearranging the steps involved in the RangeDoppler Algorithm allows the determination of target motion in the range and azimuth directions. Presented were two methods using the simple positionvelocity-acceleration time relations for the range motion detection, and a look up table based method for the azimuth motion detection. For the range direction, no post processing of the data was necessary and the motion could be determined simply using the raw data, finding instantaneous positions, and extracting velocity and acceleration information from that data. The azimuth direction required some processing, but in a different order and with less bulk than the azimuth compression used in the full RDA. Specifically, the azimuth motion detection only required the azimuth compression step without the use of range cell migration correction after, and no range compression prior. In all cases, the algorithm was successful. It was able to estimate the target's motion along either axis to within a reasonable error margin. Even in the case of the range acceleration where the error reached as high as $27 \%$ for relevant data (zeroasymptotic data excluded), it was only for acceleration values close to zero, amounting to $27 \%$ or a small number, which means a small acutal error. The code used to extract and analyze data was mostly build from scratch, without the use of pre-outside-sources code. The code itself is a collection of common operations that are also used in the RDA and shows that such algorithms can be home grown, or otherwise, invented. In terms of processing time, the methods used reduced the 
overall runtime from roughly ten seconds to a maximum of 2 seconds. Using traditional image-to-image motion detection, in which the location of the target in sequential images is tracked, motion detection would take several images to obtain an accurate number. In the case of processing time, that means ten seconds per image, which means obtaining velocity image could take upwards of twenty to thirty seconds. This thesis detects motion in about two seconds, an improvement of ten times.

This thesis is an extension of David So's and Brian Zaharri's work and advances the capabilities of the Automatic Target Recognition project's motion detection.

\section{Scope for Future Work}

The algorithm described in this paper uses collected data as a lookup table to compare the data extracted from the simulation. Essentially, the comparison is between data points along a curve. As such, the look up tables could be curve fitted with equations that could replace the comparison step of the algorithm. While this may introduce more error, it would significantly decrease the processing time - instead of comparing to thousands of data points, it would solve a handful of equations.

Another important aspect of this project is accessibility. Matlab is rwell known as a great platform on which to design and develop code; however, it is expensive. Just the bare-bones Matlab license costs $\$ 1,000$ and $\$ 30$ for each additional toolbox, such as image processing, curve fitting, etc. It is of the author's opinion 
that a useful advancement for this project would be a port to the Python scripting language. Python is a widely used language on all major platforms (linux, OS X, PC) and can be downloaded for free. Python and all of its packages, or toolboxes, are open source and downloadable for free from several sources.

A major assumption made in this paper for the azimuth motion detection is that the target was initially located in the center of the target space. The choice to center the target along the azimuth made developing the concepts of the code simpler, and was ambiguous. This simplified the code because giving the target a different initial location would appear to the algorithm as motion by the target, resulting in a less accurate or incorrect estimate. Giving the target an assumed initial condition allowed the code to know how the target had moved from that position. To make the code independent of azimuth location would be a very useful advancement on the algorithms motion detection capabilities along the azimuth. It could be a preceding algorithm that would scan a few pulse returns to detect a target, and then when the target has crossed the azimuth center, begin the SAR process and detect its motion.

As for the Range, because the raw data could be used to detect the location of the target directly, functionality to extract this information as the platform is collecting data would be a smaller, but processing time reducing improvement.

As seen in figure 16, the final target image did not present a single point, but a cluster of points that included possible rounding errors present throughout the simulation. It may be possible to alter the simulation parameters such that the 
final image represents a more focused target image. This would most likely involve and increase in resolution from the simulated system. Reducing the foot print of the target in the final image would enable the system to more easily detect different types of targets, should a multi-point target identification algorithm ever be implemented.

As was seen earlier, the azimuth compressed raw data profile images for moving targets appears jagged. The methods in this paper did not attempt to correct this issue, but it may be possible to discover the root cause of these jagged artifacts and improve the effectiveness of this method for motion detection. 


\section{REFERENCES}

1. John Schneider - Lockheed Martin. "History of RADAR" PowerPoint Presentation, Arizona State University, September 2, 2003 https://math.la.asu.edu/ kuang/LM/030902-Radar_History10.pdf

2. Wikipedia.org "History of Radar". Jan 16, 2015. http://en.wikipedia.org/wiki/History_of_radar

3. Wikipedia.org. "Synthetic Aperture Radar". January 7, 2015. http://en.wikipedia.org/wiki/Synthetic_aperture_radar

4. Cumming, Ian G., Wong, Frank H. "Digital Processing of Synthetic Aperture Radar Data”. Artech House, Inc. 2005.

5. Soumekh, Mehrdad. 'Synthetic Aperture Radar Signal Processing with MATLAB Algorithms". 1999, John Wiley \& Sons, Inc.

6. Zaharri, Brian. "TWO-DIMENSIONAL SYNTHETIC APERTURE RADAR IMAGING AND MOVING TARGET TRACKING USING THE RANGE DOPPLER ALGORITHM SIMULATED IN MATLAB” June, 2007. California Polytechnic State University, Electrical Engineering Dept.

7. So, David. "SYNTHETIC APERTURE RADAR RAPID DETECTION OF RANGE AND AZIMUTH VELOCITIES IMPLEMENTED IN MATLAB”. September 27, 2012. California Polytechnic State University, Electrical Engineering Dept.

8. Saghri, John. Private Discussions and Emails Regarding SAR Concepts. January 2012 - June 2013. 
9. Kassen, Andrew. Private Discussions and Electronic Messages Regarding Data Processing Algorithms and Statistical Data Analysis, September December 2014. 


\section{APPENDICES}

\section{APPENDIX 1: MATLAB USER GUIDE}

This section describes how to use the code. Some code belongs to Brian Zaharri, but was altered for this paper's purposes. It does not include his Kalman Filter code. Refer to the cited works section for information on Brian Zaharri's thesis paper.

\section{How to use the SAR Looping Script for Testing}

This was a simple script to run the multiple sections of code for various testing purposes. The order and operations included in testing differs from whether you are trying to detect and record motion in the range and azimuth direction. The range detection includes percent error code as the detection and comparison can be done in the same run; the azimuth direction requires first that you collect data and store it in mat files. To find the percent error in the azimuth direction, the SAR simulation is run again and the motion data is detected. The tables of data collected previously are used to compare to this newly collected data for percent error.

For this paper, eight mat files were created; three of them contained the data collected for azimuth position, velocity, and acceleration and were used for the second phase to calculate the percent error when compared to new inputs; the next five are the percent error .mat files for range velocity and acceleration, and the all three azimuth motion parameters. 
tic $\%$ the 'tic ---- toc' command measures the time it takes for code in between 'tic' and 'toc' to \% run. 'toc' is located at the end of this script.

$\%$ Input a range for the variable and the number of data points you would

$\%$ like (keep the ratio Range/data_pts rational for optimal run time)

data_pts $=1000 ; \%$ Number of data points desired

span $=100 ; \%$ This is the full range width of values over which you would like to test. This can be a range of velocity values, or acceleration values, whatever you choose to alter

Array $=$-span/2:span/data_pts:span/2-span/data_pts;

$\%$ creates an array centered around zero that spans from $<-$ span $/ 2>$ to

$<$ span/2 $>$ in a number of iterations equal to $<$ data_pts $>$.

percent_error $=$ zeros $(3$, data_pts); \%initializes the percent error table for the range direction

for loop $=1$ :data_pts,

xvel $=$ Array(loop); \% be sure to comment out these values in the SAR_Init script if you use them, otherwise the simulation will initialize them to zero every time.

SAR_Init

SAR

RMD2

$\%$ [percent error, actual value, estimated value]

percent_error $(:, l o o p)=[$ xacc_pe,$\quad$ xacc,$\quad$ xacc_estimate $]$;

disp(sprintf('xvel Percent Error: \%f', percent_error(1,loop)));

$\operatorname{disp}(\operatorname{sprintf}(' \% \mathrm{~d} / \% \mathrm{~d}$ ', loop, data_pts));

end

pe_ax $25=$ percent_error;

save('pe_ax25.mat', 'pe_ax25') \% save the collected data in a .mat file

toc $\%$ end of the 'tic-toc' command. Placing this here measures the total time for the loop to run.

This is the method for collecting data in the azimuth direction (uncommented

data_pts $=1000$;

span $=100 ; \%$ velocities from $-50 \mathrm{~m} / \mathrm{s}$ to $50 \mathrm{~m} / \mathrm{s}$

array $=-$ span/2:span/data_pts:span/2-span/data_pts;

output $=$ zeroes $(4$, PRFur $)$;

for loop $=1$ :data_pts,

yvel $=\operatorname{array}($ loop); \% Again, comment out the one you want to use in the SAR_Init script so that it is not initialized to zero. We are using velocity in this case.

$\% \quad$ yacc $=\operatorname{array}($ loop $)$;

SAR_Init

SAR

Azimuth_Compression

ACP_data_collection_script 
output $(:$, loop $)=$ data;
end

This is the method used to calculate percent error for azimuth acceleration:

data_pts $=1000$;

span $=50 ; \%$ acceleration from -25 to +25

array $=-$ span/2:span/data_pts:span/2-span/data_pts;

output $=$ zeroes $(4$, PRFur $)$;

for loop $=1$ :data_pts,

$\%$ yvel = array(loop); \% Again, comment out the one you want to use in the SAR_Init script so that it is not initialized to zero. We are using velocity in this case.

yacc $=\operatorname{array}($ loop $)$

SAR_Init

SAR

Azimuth_Compression

AMD \% outputs the data used in the next step shown below.

percent_error $=[$ xacc_pe,$\quad$ xacc,$\quad$ xacc_estimate $]$; end 


\section{SAR Initialization}

This code must be run first. It sets up all of the parameters used by the simulation.

The velocity parameters at the top of this script are vectors containing a number of points equal to $\mathrm{PRF}^{*}$ dur.

$\%$ Initial Target Range (X) and Azimuth/Cross-Range (Y) Position

$\mathrm{xn}(1)=0$;

$\mathrm{yn}(1)=0$;

$\%$ Target velocities in the $\mathrm{x}$ and $\mathrm{y}$ directions

$\mathrm{xvel}=0 ; \%$ Alter these two parameters (xvel, xacc) to give the target a constant range velocity and acceleration

xacc $=0$;

$\mathrm{vx}=$ linspace $(\mathrm{xvel}-\mathrm{xacc} / 2, \mathrm{xvel}+\mathrm{xacc} / 2, \mathrm{PRFdur})$;

yvel $=0 ; \%$ Alter these two parameters (yvel, yacc) to give the target a constant azimuth velocity and acceleration

yacc $=0$;

$\%$ vy $=$ linspace (yvel-(dur/2.*yacc),yvel+(dur/2.*yacc),PRFdur);

$\%$ General Parameters

focus $=1 ; \quad \%$ Set this flag to focus moving targets

track $=1 ; \quad \%$ Set this flag to track moving targets

noise $=0 ; \quad \%$ Set this flag to add noise to signal

ntarget $=1 ; \quad \%$ Set the number of targets

$\mathrm{cj}=\operatorname{sqrt}(-1)$;

pi $2=2 * \mathrm{pi}$

$\mathrm{c}=3 \mathrm{e} 8 ; \quad \%$ Propagation speed

$\mathrm{fc}=4.5 \mathrm{e} 9 ; \quad$ \% Carrier frequency $(4.5 \mathrm{GHz})$ (check)

$\mathrm{wc}=\mathrm{pi} 2 * \mathrm{fc}$;

lambda $=\mathrm{c} / \mathrm{fc} ; \quad \%$ Wavelength $(0.06667 \mathrm{~m})$

$\mathrm{k}=\mathrm{pi} 2 /$ lambda; $\quad \%$ Wavenumber (94.2)

$\mathrm{Vp}=200 ; \quad \%$ Velocity of platform (check)

$\mathrm{PRF}=500 ; \quad \%$ Pulse repitition frequency (check)

dur $=2 ; \quad \%$ Time of Flight (check)

PRFdur $=$ PRF $*$ dur; $\quad \%$ Speed up calculations

sta=linspace(dur/PRFdur,dur,PRFdur); \% Slow Time Array

sta=transpose(sta);

std_dev=.75; \% Standard Deviation of Noise

$\mathrm{Fn}=1 ; \quad \%$ Reflectivity

$\%$ range parameters

$\mathrm{Xc}=20000 ; \quad \%$ Range distance to center of target area (check)

$\mathrm{X} 0=200 ; \quad \%$ Target area in range is within $[\mathrm{Xc}-\mathrm{X} 0, \mathrm{Xc}+\mathrm{X} 0]$ (check)

$\mathrm{Tp}=.25 \mathrm{e}-5 ; \quad \%$ Chirp pulse duration; (check) 


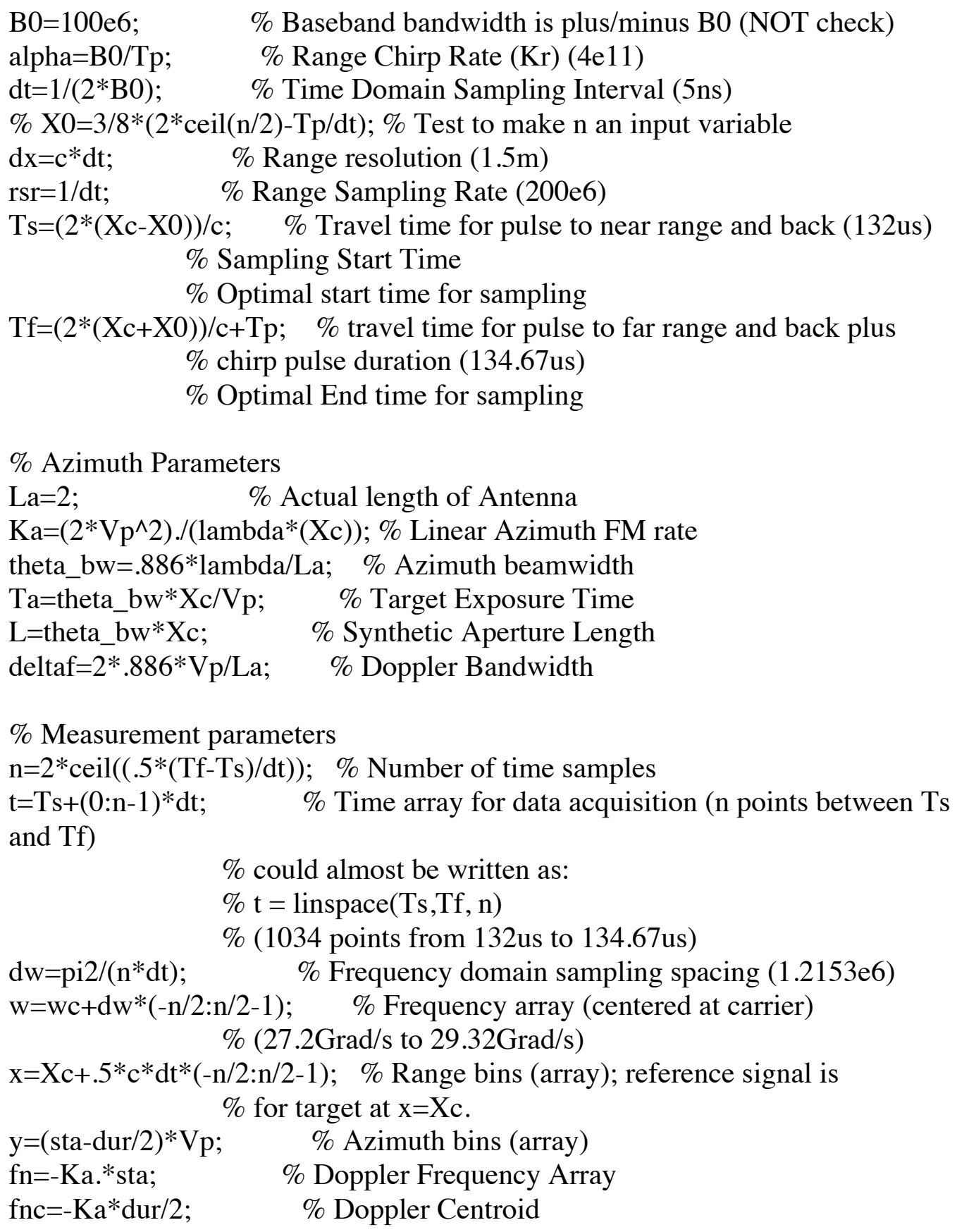




\section{Synthetic Aperture Radar Simulation}

This is the code that simulates the platform sending pulses and receiving echoes.

It results in the raw data as an image comprised of complex values. The imagesc() function plots the data ona figure in the amtlab environment. The RAW data can be viewed in multiple ways using the $\operatorname{abs}(), \operatorname{imag}(), \operatorname{real}()$ function. The code did not copy nicely over from Matlab and may need to be rearranged when pasted back in, if the actual code files are not available. Semicolons mark the ends of each line except for 'if', 'else', 'elseif', 'for' and 'end' statements (they do not require punctuation).

$\mathrm{R}=\mathrm{zeros}$ (PRFdur,ntarget); \% Initialize signal components td=zeros(PRFdur,n,ntarget); pha1=zeros(PRFdur,ntarget); pha2=zeros(PRFdur,n,ntarget); $\mathrm{s}=$ zeros $($ PRFdur,n,ntarget); rawdata=zeros(PRFdur,n);

$\%$ Initialize echoed signal array $\%$ Initialize total signal storage Array

$\%$ Process of receiving raw data based on target locations $\%$

for $\mathrm{j}=1$ :(PRFdur); $\quad \%$ Number of pulses in total time of flight $\mathrm{s} 2=\mathrm{zeros}(1, \mathrm{n})$; for $\mathrm{i}=1$ :ntarget; $\quad \%$ Gets a return from each target $\mathrm{wa}=\operatorname{sinc}(\mathrm{La} *(\operatorname{atan}(\mathrm{Vp} *(\operatorname{sta}-$ $((\operatorname{dur} / 2)+(\operatorname{yn}(\mathrm{i}) / \mathrm{Vp})+(\operatorname{vy}(\mathrm{i}, \mathrm{j}) * \mathrm{sta}) / \mathrm{Vp})) / \mathrm{Xc})) /$ lambda $){ }^{\wedge} 2 ; \%$ Azimuth beam pattern $\mathrm{R}(\mathrm{j}, \mathrm{i})=\operatorname{sqrt}\left(\left(\mathrm{Xc}+\mathrm{xn}(\mathrm{i})+\operatorname{sta}(\mathrm{j}) \cdot{ }^{*} \mathrm{vx}(\mathrm{i}, \mathrm{j})\right)\right)^{\wedge} 2+\left(\mathrm{Vp}^{\wedge} 2^{*}((\operatorname{sta}(\mathrm{j})-\right.$ $((\operatorname{dur} / 2)+(\mathrm{yn}(\mathrm{i}) / \mathrm{Vp})+(\mathrm{vy}(\mathrm{i}, \mathrm{j}) * \mathrm{sta}(\mathrm{j})) / \mathrm{Vp})) . \wedge 2))) ; \%$ Instantaneous Slant Range $\operatorname{td}(\mathrm{j}, ;, \mathrm{i})=\mathrm{t}-2 * \mathrm{R}(\mathrm{j}, \mathrm{i}) / \mathrm{c} ; \%$ time calculation that subtracts instantaneous pulse travel time from start time of sampling for FM calculations to follow pha $1(\mathrm{j}, \mathrm{i})=4 * \mathrm{pi}^{*}(\mathrm{fc} / \mathrm{c}) * \mathrm{R}(\mathrm{j}, \mathrm{i}) ; \%$ First FM term in the phase power series pha2 $(\mathrm{j}, ;, \mathrm{i})=\mathrm{pi}^{*}$ alpha* $\left.\left((\operatorname{td}(\mathrm{j},:, \mathrm{i}))^{\wedge} 2\right)-\operatorname{td}(\mathrm{j}, ;, \mathrm{i}) * \mathrm{Tp}\right) ; \%$ second $\mathrm{FM}$ term in the phase power series [all other power series terms (for $\mathrm{n}>2)]=0$ ) $\mathrm{s}(\mathrm{j},:, \mathrm{i})=\mathrm{s}(\mathrm{j},: \mathrm{i})+\mathrm{Fn} * \mathrm{wa}(\mathrm{j}) * \exp (-\mathrm{cj} * \operatorname{pha1}(\mathrm{j}, \mathrm{i}))^{*} \exp (\mathrm{cj} * \operatorname{pha} 2(\mathrm{j},:, \mathrm{i})) *(\operatorname{td}(\mathrm{j},:, \mathrm{i})>=0$ $\& \operatorname{td}(\mathrm{j},:, \mathrm{i})<=\mathrm{Tp}) ; \%$ Signal pulse - Initialized as a matrix of zeros if noise $==1$ $s(\mathrm{j},:, \mathrm{i})=\mathrm{s}(\mathrm{j},:, \mathrm{i})+\operatorname{std} \_$dev*${ }^{*} \operatorname{randn}(\operatorname{size}(\mathrm{s}(\mathrm{j}, ; \mathrm{i}))) ; \%$ adding noise to the signal end;

$\mathrm{s} 2=\mathrm{s} 2+\mathrm{s}(\mathrm{j},: \mathrm{i}) ; \quad \%$ Store each targets return into intermediate signal end;

rawdata $(\mathrm{j},:)=\mathrm{s} 2 ;$ end; $\quad \%$ Stores each echo into s3 (raw data vectors) 


\section{Range Motion Detection}

This code is run directly after the SAR simulation to determine Range motion detection. Comments are written throughout.

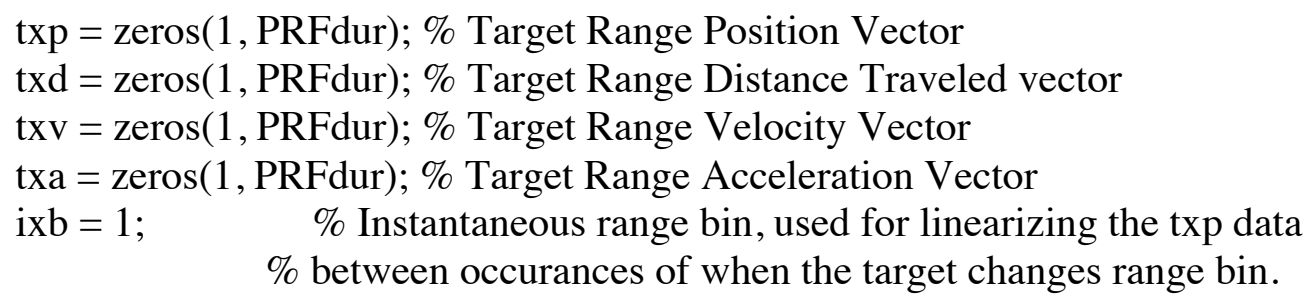

$\operatorname{txp}(1)=$ floor $($ mean $($ find $(\operatorname{abs}(\operatorname{rawdata}(1,:)))))-\mathrm{n} / 2-1 ; \%$ Initialized

for $\mathrm{i}=2$ :PRFdur,

$\operatorname{txp}(\mathrm{i})=$ floor $(\operatorname{mean}(\operatorname{find}(\operatorname{abs}(\operatorname{rawdata}(\mathrm{i},:)))))-\mathrm{n} / 2-1$;

if $(\operatorname{txp}(\mathrm{i}) \sim=\operatorname{txp}(\mathrm{i}-1)) \%$ This is why the code was initialized (The i-1 $\%$ term would result in a 0 index, a Matlab $\%$ no-no.

$\%$ txd is created from linearizing the first occurance of the previous

$\%$ range bin with the occurance of a new range bin. It essentially smooths

$\%$ the txp data from being discrete to continuous.

$\operatorname{txd}(\mathrm{ixb}: \mathrm{i}-1)=$ linspace $(\operatorname{txp}(\mathrm{ixb}), \operatorname{txp}(\mathrm{i}),(\mathrm{i}-\mathrm{ixb}))-\operatorname{txp}(1)$;

$\mathrm{ixb}=\mathrm{i} ; \%$ Update the range bin.

end

end

txd(ixb:PRFdur $)=$ linspace $(\operatorname{txp}(\mathrm{ixb}), \operatorname{txp}(\mathrm{PRFdur}), \mathrm{PRFdur}+1$ - ixb $)-\operatorname{txp}(1)$;

txv = txp./sta.'; \% Time division to make velocity from positions

txa = txv./sta.'; \% Second time division to make acceleration from velocity (unused)

av_txp $=$ mean $(\mathrm{txd}) *(\mathrm{dx} /(\mathrm{Vp}) * 100) ; \%$ Convert position to meters from bins $)$ $\mathrm{dxdt}=\operatorname{diff}(\operatorname{txp}) ; \% \mathrm{x}(\mathrm{n}+1)-\mathrm{x}(\mathrm{n})=\mathrm{v}(\mathrm{n})$, change in range position av_txv $=$ mean $(\mathrm{dxdt}) *(\mathrm{dx} /(\mathrm{Vp} / \mathrm{PRF}) * 100) ; \%$ Convert velocity to meters from bins $\mathrm{dvdt}=\operatorname{diff}(\mathrm{txv}) ; \%$ sample to sample $\mathrm{v}(\mathrm{n}+1)-\mathrm{v}(\mathrm{n})=\mathrm{a}(\mathrm{n})$, change in range velocity

av_txa $=$ mean $(\operatorname{dvdt}(\mathrm{PRFdur} / 5: \mathrm{PRFdur}-2)) *(\mathrm{dx} /(\mathrm{Vp} / \mathrm{PRF}) * 100) ; \%$ Convert acceleration from meters to bins

$\%$ These if statements rule out the possibility of a NaN case (isnan()

$\%$ checks for this)

if isnan(av_txv) II av_txv $==0$,

xvel_estimate $=0$;

xvel_pe $=0$;

else

xvel_estimate $=$ av_txv;

xvel_pe $=($ xvel - xvel_estimate $) . / x v e l * 100$; 


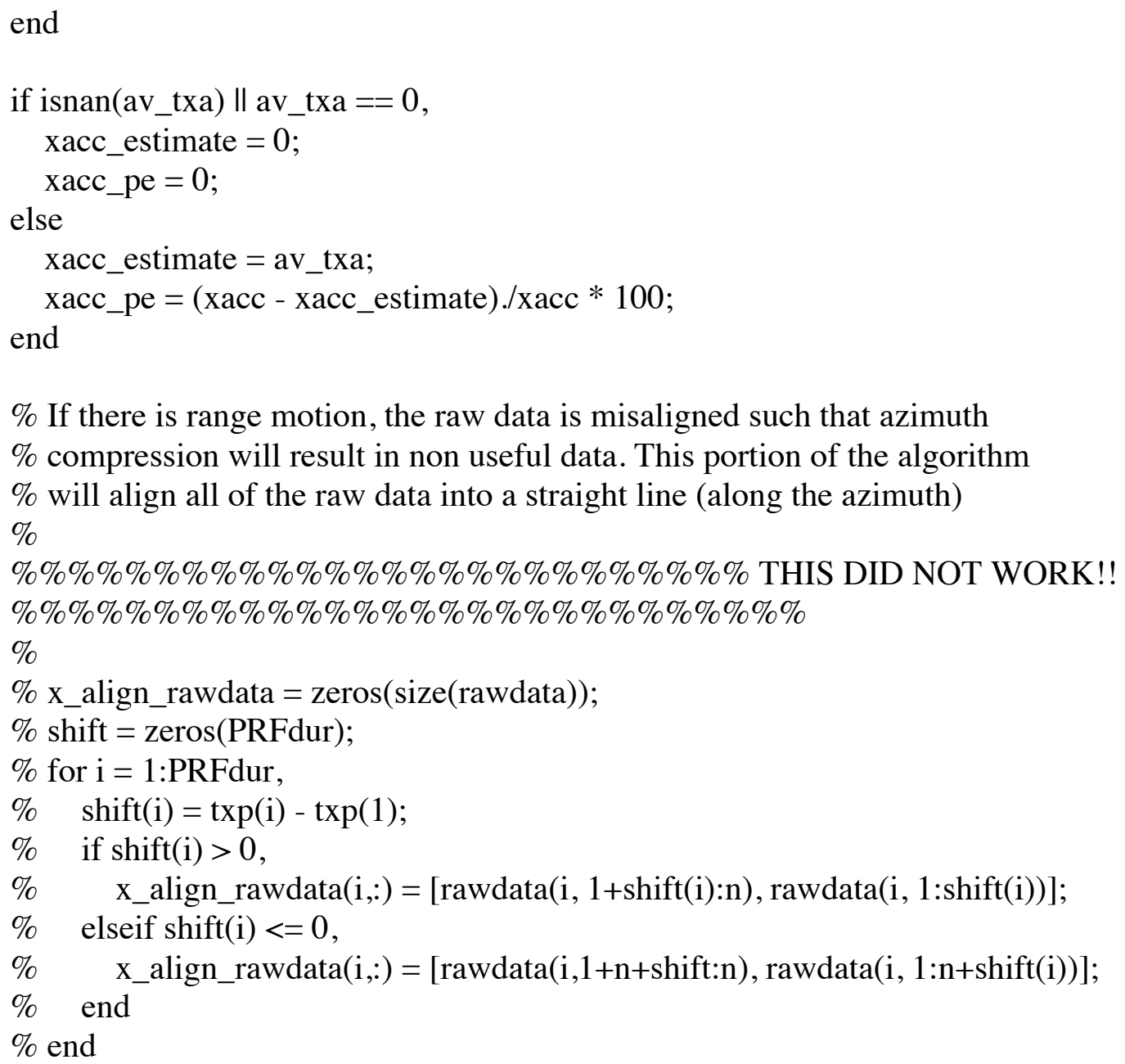




\section{Azimuth Compression}

In this code RCMC is commented, but can be uncommented and used. In order to change the input to this function, simply change the name written in the ftx function to create the variable called 'fsmb'. It is currently written as 'rawdata'.

$\%$ function [azimuth_compressed_data] = Azimuth_Compression(input_data) ref $=$ cj*pi*Ka. ${ }^{*}$ sta. $*(2 *$ sta(PRFdur/2+1)-sta);

$\mathrm{smb} 0=\exp ($ ref $) ; \% \operatorname{smb0}=\cos ($ ref $)+\mathrm{j}^{*} \sin ($ ref $)$;

$\mathrm{fsmb} 0=\mathrm{ftx}(\mathrm{smb}) ; \%$ Azimuth matched filter spectrum

fsac0 $=$ fsmb0 $*$ conj(fsmb0);

$\operatorname{sac} 0=\operatorname{iftx}($ fsac0 $)$;

$\%$ Azimuth compression done for each range bin

$\mathrm{fsmb}=\mathrm{ftx}($ rawdata $) ; \quad \%$ Azimuth Fourier Transform

fsac $=\operatorname{zeros}($ PRFdur, $\mathrm{n})$;

$\%$ deltaR $=\left(\right.$ lambda $\left.{ }^{\wedge} *(\mathrm{Xc}) . *(\mathrm{fn}-\mathrm{fnc}) . \wedge 2\right) /\left(8 * \mathrm{Vp}^{\wedge} 2\right) ; \%$ Range Cell Migration in meters

$\%$ cells=round(deltaR/.56); $\quad \% .56$ meters/cell in range direction

$\%$

$\% \%$ Range cell migration correction done for first half of signals

$\% \%$ then mirrored for second half (symmetrical)

$\%$ for $\mathrm{k}=1: \mathrm{dur} * \mathrm{PRF} / 2$

$\%$ for $m=1: n-9$

$\% \quad \mathrm{fsmb}(\mathrm{k}, \mathrm{m})=\mathrm{fsmb}(\mathrm{k}, \mathrm{m}+\mathrm{cell}(\mathrm{k}))$; \%Range Cell Migration Correction

$\% \quad$ fsmb(PRFdur-k,m)=fsmb(PRFdur-k,m+cells(k));

$\%$ end

$\%$ end;

for $1=1: n$,

fsac $(:, 1)=f \operatorname{smb}(:, 1){ }^{*} \operatorname{conj}(\mathrm{fsmb} 0) ; \%$ Azimuth Compression end

azimuth_compressed_data $=$ iftx(fsac); $\quad \%$ Azimuth Inverse Fourier

Transform

$\%$ imagesc(abs(azimuth_compressed_data)); 


\section{Azimuth Compression Profile Data Collection}

This code is used in the data collection phase for the azimuth direction.

$\%$ sac $=$ Azimuth Compressed Data

$\mathrm{ACP}=\max ($ transpose $(\operatorname{abs}($ azimuth_compressed_data $))) ; \%$ Azimuth

Compression Profile

$\mathrm{ACPmean}=\operatorname{mean}(\mathrm{ACP})$;

$\mathrm{ACPmax}=\max (\mathrm{ACP})$

ACPsum = zeros(1, PRFdur $)$;

for $\mathrm{i}=1$ :PRFdur,

$\operatorname{ACPsum}(1, i)=\operatorname{sum}(\operatorname{ACP}(1,1: i))$

end

$\%$ sde $=$ signal density estimate, The total sum of the signal magnitudes

$\%$ (the sum of elements from the Azimuth Compression Maxima Profile

$\%$ centered around the SDE.

[dontcare, $\mathrm{ACPsde}]=\min (\operatorname{abs}(\operatorname{ACPmean} * \operatorname{PRFdur} / 2-\operatorname{ACPsum}(1,:)))$;

$\mathrm{ACPsize}=\operatorname{size}(\mathrm{ACP}(\mathrm{ACP}>\mathrm{ACPmax} * 0.1), 2)$;

data $=[$ ACPmean, ACPmax, ACPsde, ACPsize $]$; 


\section{Azimuth Motion Detection}

This code should be run after the azimuth compression IF and ONLY IF there was no range compression. If there was range compression, there is no guarantee that this code will work. It uses the output of the preceding azimuth compression code. This script is used after the ACP data has been collected and stored in .mat files. The files AMD_5220_yn190.mat,AMD_5220_vy50,AMD_5220_ay25 are named based on specific parameters and what was varied. Looking at the first file name, 'AMD' stands for Azimuth motion detection; the '5220' shows a PRF of 500 , a duration of 2 seconds, and a range of closest approach of $20 \mathrm{~km}$; the 'yn190' means that for each iteration, the position of the target was varied from 190 to 190; for the other filenames, vy and ay stand for azimuth velocity and acceleration, respectively. These files contain the data collected during the azimuth data collection phase.

$\%$ sac $=$ Azimuth Compressed Data

$\mathrm{ACP}=\max (\operatorname{transpose}(\operatorname{abs}($ azimuth_compressed_data $))) ; \%$ Azimuth

Compression Profile

ACPmean $=\operatorname{mean}(\mathrm{ACP})$;

$\mathrm{ACPmax}=\max (\mathrm{ACP})$

ACPsum = zeros(1, PRFdur);

for $\mathrm{i}=1$ :PRFdur,

$\operatorname{ACPsum}(1, i)=\operatorname{sum}(\operatorname{ACP}(1,1: i))$;

end

$\%$ sde $=$ signal density estimate, The total sum of the signal magnitudes

$\%$ (the sum of elements from the Azimuth Compression Maxima Profile

$\%$ centered around the SDE.

$[\mathrm{dc}$, ACPsde $]=\min (\operatorname{abs}($ ACPmean*PRFdur/2 $-\operatorname{ACPsum}(1,:)))$;

$\mathrm{ACPsize}=\operatorname{size}(\mathrm{ACP}(\mathrm{ACP}>\mathrm{ACPmax} * 0.1), 2)$;

data $=[$ ACPmean, ACPmax, ACPsde, ACPsize $]$;

yn_estimate $=$ zeros $(4,2) ; \%$ yn_estimate_I $=$ zeros $(4)$;

vy_estimate $=$ zeros $(4,2) ; \%$ vy_estimate_I $=$ zeros $(4)$;

ay_estimate $=$ zeros $(4,2) ; \%$ ay_estimate_I $=$ zeros $(4)$; 
estimate $=0 ; \%$ No estimate made

$\%$ max of stationary width plot (PRFdur/20),

$\%$ plus $20 \%(\mathrm{PRFdur} / 20 * 1.2=\mathrm{PRFdur} * 0.06$

if ACPsize < PRFdur*0.06, \% This is the limitation on azimuth bin width.

$\%$ Anything thinner than PRF*constant is assumed

$\%$ to be a stationary target

approx_loc = AMD_5220_yn190(5,ACPsde); \% AMD_5220_yn190 is the name

approx_yvel =0;

$\%$ of the file containing all

$\%$ stationary target data along

$\%$ the azimuth axis and is used

$\%$ as a lookup table

approx_yacc $=0$;

loc_percent_err $=($ yloc - approx_loc $) / y l o c * 100$;

vel_percent_err $=100$;

acc_percent_err = 100;

disp(sprintf('Target is traveling less than $\left.\left.3.5 \mathrm{~m} / \mathrm{s}^{\prime}\right)\right)$;

disp(sprintf('Target approximate location: \%f m', approx_loc));

estimate $=1 ; \%$ estimate made

elseif ACPsde - PRFdur $/ 2<=0, \%$ To the left of the Azimuth Center (negative)

for $i=1: 4 \%$ [1. mean, 2. max, 3. approx_location, 4. width of signal]

$\%$ Takes the least squared error between extracted data and lookup

$\%$ table files. The 5220 stands for PRF of 500, duration of 2

$\%$ seconds, at am Xc of $20 \mathrm{~km}$. 5220. AMD stands for Azimuth Motion

$\%$ Detection

[yn_estimate $(\mathrm{i}, 1), \mathrm{yn} \_$estimate $\left.(\mathrm{i}, 2)\right]=$

$\min \left(\operatorname{abs}\left(A M D \_5220 \_y n 190(\mathrm{i}, 1: P R F d u r / 2) . \wedge 2\right.\right.$ - data(i).^2));

[vy_estimate(i,1),vy_estimate $(\mathrm{i}, 2)]=$

$\min \left(\operatorname{abs}\left(A M D \_5220 \_v y 50(\mathrm{i}, 1: P R F d u r / 2) . \wedge 2\right.\right.$ - data(i).^2));

[ay_estimate(i,1),ay_estimate $(\mathrm{i}, 2)]=$

$\min \left(\operatorname{abs}\left(A M D \_5220 \_a y 25(i, 1: P R F d u r / 2) . \wedge 2\right.\right.$ - data(i).^2));

end

elseif ACPsde - PRFdur $/ 2>0, \%$ To the right of the azimuth center (positive)

for $i=1: 4 \%$ [1. mean, 2. max, 3. approx_location, 4. width of signal]

[yn_estimate $(\mathrm{i}, 1), \mathrm{yn} \_$estimate $\left.(\mathrm{i}, 2)\right]=$

$\min \left(\operatorname{abs}\left(A M D \_5220 \_y n 190(\mathrm{i}, P R F d u r / 2+1: P R F d u r) . \wedge 2\right.\right.$ - data(i).^2));

[vy_estimate $\left.(i, 1), v y \_e s t i m a t e(i, 2)\right]=$

$\min (\operatorname{abs}($ AMD_5220_vy50(i,PRFdur/2+1:PRFdur).^2 - data(i).^2));

[ay_estimate(i,1),ay_estimate $(\mathrm{i}, 2)]=$

min(abs(AMD_5220_ay25(i,PRFdur/2+1:PRFdur).^2 - data(i).^2));

yn_estimate $(\mathrm{i}, 2)=$ yn_estimate $(\mathrm{i}, 2)+\mathrm{PRFdur} / 2$;

vy_estimate $(\mathrm{i}, 2)=$ vy_estimate $(\mathrm{i}, 2)+\mathrm{PRFdur} / 2$;

ay_estimate $(\mathrm{i}, 2)=$ ay_estimate $(\mathrm{i}, 2)+\mathrm{PRFdur} / 2$;

end 
end

if estimate $==0, \%$ Estimate not yet made

loc_percent_err $=0$;

vel_percent_err $=0$;

acc_percent_err $=0$;

$\%$ Finds the best option from each lookup table by summing up the errors

$\%$ from each. The least summed square error is the best estimate, no?

[best_estimate, best_index] $=\min \left(\left[\operatorname{sum}\left(y n \_e s t i m a t e(1: 3,1) .^{\wedge} 2\right)\right.\right.$, $\operatorname{sum}\left(\right.$ vy_estimate $\left.(:, 1) .^{\wedge} 2\right), \operatorname{sum}($ ay_estimate $\left.\left.(:, 1) . \wedge 2)\right]\right)$;

if best_index $==1, \%$ Stationary Target. This should never happen, see previous if statements

$\%$ This is redundant.

approx_loc $=$

round(AMD_5220_yn190(5,round(mean(yn_estimate $(1: 3,2)))))$;

if approx_loc $\sim=0$,

loc_percent_err $=($ yloc - approx_loc $) / y l o c * 100$;

end

$\operatorname{disp}\left(\operatorname{sprintf}\left('\right.\right.$ Target is traveling less than $\left.3.5 \mathrm{~m} / \mathrm{s}^{\prime}\right)$ );

disp(sprintf('Target approximate location: \%f m', approx_loc));

elseif best_index $==2, \%$ Constant velocity target

approx_yvel = AMD_5220_vy50(5,round(mean(vy_estimate $(:, 2))))$;

if yvel $\sim=0$,

vel_percent_err $=($ yvel - approx_yvel $) / y v e l * 100$;

end

disp(sprintf('Target is traveling with approximate velocity of $\% \mathrm{f} \mathrm{m} / \mathrm{s}$, approx_yvel));

elseif best_index $==3, \%$ Accelerating target

approx_yacc $=$ AMD_5220_ay25(5,round $($ mean(ay_estimate $(:, 2))))$;

if yacc $\sim=0$,

acc_percent_err $=($ yacc - approx_yacc $) /$ yacc $* 100$;

end

$\operatorname{disp}(\operatorname{sprintf}($ 'Target is traveling with approximate acceleration of $\% \mathrm{f} \mathrm{m} / \mathrm{s} / \mathrm{s}$, approx_yacc))

end

end 


\section{Power Equalization and Range Compression}

The input to this code is changed by altering the variable in the fty function in the 'for' loop below (currently written as rawdata).

$\%$ Reference Signal for range compression $\mathrm{td} 0=\mathrm{t}-2 *(\mathrm{Xc} / \mathrm{c})$

pha20 $=$ pi*alpha* $((\operatorname{td} 0 . \wedge 2)-\operatorname{td} 0 * \mathrm{Tp})$;

$\mathrm{s} 0=\exp \left(\mathrm{cj}^{*} \mathrm{pha} 20\right) *(\mathrm{td} 0>=0 \& \mathrm{td} 0<=\mathrm{Tp})$;

$\mathrm{fs} 0=\mathrm{fty}(\mathrm{s} 0) ; \quad \%$ Reference Signal in frequency domain

$\%$ Power equalization

$\operatorname{mag}=\operatorname{abs}(\mathrm{fs} 0)$

amp_max=1/sqrt(2); \% Maximum amplitude for equalization

afsb0 $=$ abs (fs0);

P_max $=\max (\mathrm{afsb0})$;

$\mathrm{I}=$ find(afsb0 $>=$ amp_max*P_max); \% Finds where the amp. of afsb0 is

$\% 1 /$ sqrt(2)than the max power

nI=length $(\mathrm{I})$;

fs0(I) $=\left(\left(\operatorname{amp} \_m a x *\left(P \_m a x{ }^{\wedge} 2\right) * \operatorname{ones}(1, \mathrm{nI})\right) \cdot / \operatorname{afsb0}(\mathrm{I})\right) \cdot * \exp \left(\mathrm{cj}^{*}\right.$ angle(fs0(I)));

$\mathrm{E}=\operatorname{sum}(\mathrm{mag} . * \operatorname{abs}(\mathrm{fs} 0))$;

$\%$ Initialize Range Compression Arrays

fs=zeros $($ PRFdur, $n)$;

fsm=zeros(PRFdur,n);

$\mathrm{smb}=$ zeros(PRFdur, $\mathrm{n})$;

$\%$ Range compression done for each pulse

for $\mathrm{k}=1$ :(PRFdur);

$\mathrm{fs}(\mathrm{k},:)=\mathrm{fty}(\operatorname{rawdata}(\mathrm{k},:))$;

$\mathrm{fsm}(\mathrm{k},:)=\mathrm{fs}(\mathrm{k},:){ }^{*} \operatorname{conj}(\mathrm{fs} 0) ; \quad \%$ Matched Filter Multiplication in frequency domain

$\operatorname{smb}(\mathrm{k},:)=\mathrm{ifty}(\mathrm{fsm}(\mathrm{k},:)) ; \quad \%$ Matched filtered signal (range reconstruction) end; 


\section{Offline Processing and Compensation}

This code was unused for this paper, but it is one of the original pieces of the RDA presented in Brian Zaarri's work.

$\%$ Offline Processing and compensation of moving targets

$\%$

$\%$ if movement $==1 \& \&$ focus $==1$

$\% \quad \mathrm{Rc}=$ zeros(PRF*dur,ntarget $)$; Initialize slant range compensation array

$\%$ for $\mathrm{j}=1:\left(\mathrm{PRF}^{*} \mathrm{dur}\right)$;

$\% \quad \mathrm{~s} 2=\mathrm{zeros}(1, \mathrm{n})$

$\%$ for $\mathrm{i}=1$ :ntarget;

$\% \quad$ if flag(i)==1 $\%$ Will only compensate for targets that are moving

$\%$

$\% \operatorname{needed}(\mathrm{j})=\operatorname{sqrt}\left(\left((\operatorname{dur} / 2-\operatorname{sta}(\mathrm{j})) * \operatorname{Vp}+\operatorname{mean}(\operatorname{yvel}(:, \mathrm{i}))^{*} \operatorname{sta}(\operatorname{dur} * \mathrm{PRF})\right) .{ }^{\wedge} 2\right.$

$\left.+\mathrm{Xc}^{\wedge} 2\right)$

$\% \quad \operatorname{actual}(\mathrm{j})=\operatorname{sqrt}\left(((\operatorname{dur} / 2-\operatorname{sta}(\mathrm{j})) * \mathrm{Vp}+\operatorname{mean}(\operatorname{yvel}(:, \mathrm{i})) * \operatorname{sta}(\mathrm{j})) .^{\wedge} 2+\mathrm{Xc}^{\wedge} 2\right)$

$\% \quad \operatorname{Rc}(\mathrm{j}, \mathrm{i})=\operatorname{mean}(\operatorname{xvel}(:, \mathrm{i})) *(\operatorname{dur}-\mathrm{sta}(\mathrm{j}))+(\operatorname{needed}(\mathrm{j})-\operatorname{actual}(\mathrm{j})) ; \%$ Compensated range

$\% \quad \% \operatorname{Rc}(\mathrm{j}, \mathrm{i})=\operatorname{xpos}((\operatorname{dur} * \mathrm{PRF}+1)-\mathrm{j}, \mathrm{i})+(\operatorname{needed}(\mathrm{j})-\operatorname{actual}(\mathrm{j})) ; \quad \%$ Other ideas

$\% \quad \mathrm{tdc}=\mathrm{t}-2 *(\mathrm{R}(\mathrm{j}, \mathrm{i})+\mathrm{Rc}(\mathrm{j}, \mathrm{i})) / \mathrm{c}$;

$\% \quad$ phalc $=0$;

$\% \quad$ if ymove $(\mathrm{i})==1$

$\% \quad$ pha1c $=4 *$ pi* $(\mathrm{fc} / \mathrm{c}) * \operatorname{Rc}(\mathrm{j}, \mathrm{i})$;

$\% \quad$ end;

$\% \quad$ pha2c=pi*alpha*((tdc.^2)-tdc*Tp);

$\% \quad \mathrm{~s}(\mathrm{j},:, \mathrm{i})=\mathrm{s}(\mathrm{j},:, \mathrm{i}) * \exp (-\mathrm{cj} * \mathrm{pha1c}) . * \exp (-$

$\mathrm{cj}^{*}$ pha2 $\left.(\mathrm{j},:, \mathrm{i})\right) . * \exp (\mathrm{cj} *$ pha2c).*(tdc $>=0 \&$ tdc $<=\mathrm{Tp}) ; \%$ New signal vector $\% \quad$ end;

$\% \quad \mathrm{~s} 2=\mathrm{s} 2+\mathrm{s}(\mathrm{j},:, \mathrm{i})$

$\% \quad$ end;

$\% \quad \mathrm{~s} 3(\mathrm{j},:)=\mathrm{s} 2 ; \quad \%$ New raw data array

$\%$ end;

$\%$ end; 


\section{APPENDIX 2: SAR EXAMPLE}

\section{Synthetic Aperture Explained}

Imagine a tank sitting in a field 20 kilometers north of point A and an Airborne SAR system at point B, 300 meters west of point A, cruising along a straight path at 200 meters per second with a constant altitude. The radar system begins sending and receiving pulses at a PRF of $300 \mathrm{~Hz}$. The platform continues moving and collecting data, and passes over point A. This point during capture is known as the Range of Closest approach, $X_{c}$, and represents the distance from the platform when the target lies in the zero Doppler plane, from which the radar sees a zero Doppler shift. Int his case, $X_{c}$ is the distance from point $A$ to the tank, 20,000 meters. The platform continues collecting data until it reaches point C, 300 meters east of point A. During its flight time from point B to point $\mathrm{C}$, the platform accumulated data from 900 pulses over a distance of 600 meters. Although the actual antenna size for the radar platform is relatively small, the effective antenna size, or the synthetic aperture size, for all of the data collected is much larger. Calculation for the length of the synthetic aperture is as follows:

Assuming the carrier frequency of the system to be:

$$
f_{c}=4.5 \mathrm{GHz}
$$

Using the equation for the speed of light we can calculate the carrier wavelength of the system,

$$
\lambda=\frac{c}{f}
$$




$$
\begin{gathered}
\lambda=\frac{3.0 * 10^{8} \mathrm{~m} / \mathrm{s}}{4.5 * 10^{9} \mathrm{~Hz}} \\
\lambda=0.0667 \mathrm{~m}
\end{gathered}
$$

For an actual antenna length of,

$$
L_{a}=2 \mathrm{~m}
$$

It follows that the cross-range, or Azimuth, beamwidth from the radar system is,

$$
\begin{aligned}
& \theta_{b w}=\frac{0.886 * \lambda}{L_{a}} \\
& \theta_{b w}=0.02953
\end{aligned}
$$

And finally, the synthetic aperture length is,

$$
\begin{gathered}
L_{s}=\theta_{b w} * X_{c} \\
L_{s}=590.667 \mathrm{~m}
\end{gathered}
$$

This figure shows the obvious advantage of using synthetic aperture over traditional radar. While a real antenna length of 590 meters is practically infeasible, it is a typical synthetic aperture length in applications such as this one. Impressively, Spaceborne SAR systems can achieve synthetic apertures of thousands or even tens of thousands of meters long for capturing sub-meter resolutions with ranges surpassing 20,000 kilometers. 